Renata Goldman Leibel

Fiber Monitoring Technique with Sub-carrier Baseband Tone Sweep

Thesis presented to the Programa de Pós-graduação em Engenharia Elétrica of PUC-Rio in partial fulfillment of the requirements for the degree of Doutor em Engenharia Elétrica.

Advisor: Prof. Jean Pierre von der Weid 

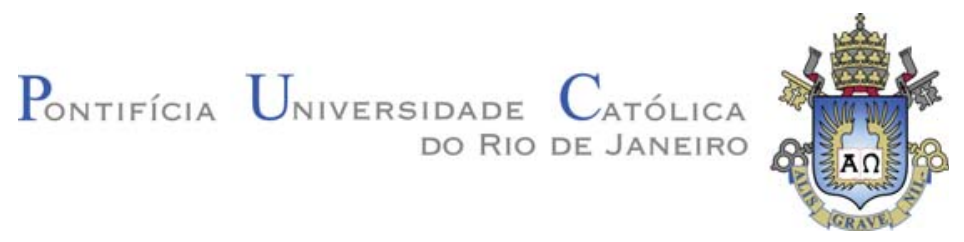

Renata Goldman Leibel

\section{Fiber Monitoring Technique with Sub-carrier Baseband Tone Sweep}

Thesis presented to the Programa de Pós-graduação em Engenharia Elétrica of PUC-Rio in partial fulfillment of the requirements for the degree of Doutor em Engenharia Elétrica. Approved by the undersigned Examination Committee.

Prof. Jean Pierre von der Weid

Advisor

Centro de Estudos e Telecomunicações - PUC-Rio

Prof. Guilherme Penello Temporão

Centro de Estudos e Telecomunicações - PUC-Rio

Prof. Luis Ernesto Ynoquio Herrera

Centro de Estudos e Telecomunicações - PUC-Rio

Prof. Andrew Henry Cordes

Centro de Estudos e Telecomunicações - PUC-Rio

Prof. Ricardo Marques Ribeiro

UFF

Prof. Giancarlo Vilela de Faria

Ouro Negro SA

Profa. Maria Aparecida Gonçalves Martinez

CEFET/RJ

Prof. Márcio da Silveira Carvalho

Vice Dean of Graduate Studies Centro Técnico Científico- PUC-Rio 
All rights reserved.

\section{Renata Goldman Leibel}

The author got her bachelor's degree in Electrical Engineering from PUC-Rio in 2005, and her master's degree in Electrical Engineering from The Technion - Israel Institute of Technology in 2013, in optical communications and fiber monitoring. As part of the doctorate, she collaborated on a series of projects with Ericsson Research, Sweden.

Bibliographic data

Leibel, Renata Goldman

Fiber Monitoring Technique with Sub-carrier Baseband Tone Sweep / Renata Goldman Leibel; advisor: Jean Pierre von der Weid. - 2018.

$101 \mathrm{f}$ : il. color. ; $30 \mathrm{~cm}$

Tese (doutorado) - Pontifícia Universidade Católica do Rio de Janeiro, Departamento de Engenharia Elétrica, 2018.

Inclui bibliografia

1. Engenharia Elétrica - Teses. 2. Monitoramento de Fibras Ópticas. 3. Reflectometria Óptica no Domínio do Tempo. 4. Reflectometria Óptica no Domínio da Frequência. 5. Monitoramento de Fibras em Serviço. 6. Multiplexação por Sub-portadoras.

I. Weid, Jean Pierre von der. II. Pontifícia Universidade Católica do Rio de Janeiro. Departamento de Engenharia Elétrica. III. Título. 
To my parents, for their support and encouragement. To my husband, for his patience. To my son. 


\section{Acknowledgments}

I would like to first thank my advisor for his patience and time in the lab. His insights were very important in achieving the results of this work as well as all through the path of projects that led to it.

Next, I wish to thank my colleagues at the CETUC Opto-electronics laboratory. A special thanks to my friend Luis, who helped me with setup and splicing fiber, taught me a lot and helped me learn my way around the lab equipment. I would also like to thank Diego and Andrea who took part in related work which partially led to the results presented here. Their help was indispensable for me to reach the goals proposed in this thesis.

Furthermore, I would like to thank Ericsson Research, CNPq, the department of Electrical Engineering at PUC-Rio and CETUC for financial support.

Finally, I wish to thank my family, my parents and my husband, who cheered me and supported me in more ways than I can count so I could reach my goals. 


\section{Abstract}

Leibel, Renata Goldman; Weid, Jean Pierre von der (Advisor). Fiber Monitoring Technique with Sub-carrier Baseband Tone Sweep. Rio de Janeiro, 2018. 101p. Tese de doutorado - Departamento de Engenharia Elétrica, Pontifícia Universidade Católica do Rio de Janeiro.

With ever growing demand for higher capacity in data transmission, a solution has been proposed for analog Radio over Fiber transmission, combining several data channels over the optical carrier in the access network using Sub-Carrier Multiplexing (SCM). Furthermore, this access network is often branched in the form of a passive optical network (PON). Monitoring such fiber links is thus increasingly important. Current optical time domain reflectometry (OTDR) technology used for monitoring operates at a separate optical wavelength and has wide bandwidth detection, making it relatively costly. The pursuit of a low cost, high precision monitoring alternative is of paramount importance for cost effective quality of service (QoS) improvement. In this work employment of SCM for data multiplexing is exploited embedding a low frequency tone sweep monitoring technique into data transmission. A mathematical model for the frequency response of the link was derived and the fault location and intensity is estimated through comparison with acquired amplitude and phase of the backscattered light for the varying frequency by applying a simple least mean squared (LMS) algorithm. The modeling suggested requires that measurement of the link prior to the occurrence of a fault is known. A single new fault can then be accurately detected and its position and intensity estimated in the way described.

\section{Keywords}

Optical Fiber Monitoring; Optical Time Domain Reflectometer; Optical Frequency Domain Reflectometer; In service Fiber Monitoring; Subcarrier Multiplexing; Passive Optical Networks; 5G Transport Networks; Physical Layer Monitoring 


\section{Resumo}

Leibel, Renata Goldman; Weid, Jean Pierre von der. Monitoramento de Fibras Ópticas por Varredura de Tom na SubPortadora de Banda Base. Rio de Janeiro, 2018. 101p. Tese de Doutorado - Departamento de Engenharia Elétrica, Pontifícia Universidade Católica do Rio de Janeiro.

Com a crescente demanda por maior capacidade na transmissão de dados, uma solução tem sido estudada para transmissão de rádio sobre fibra combinando múltiplos canais de dados sobre a portadora óptica na rede de acesso baseada em multiplexação por sub-portadoras. Ademais, essa rede de acesso é comumente ramificada na forma de uma rede óptica passiva. Monitoramento desses enlaces em fibra é, então, cada vez mais importante. A tecnologia atual de reflectometria óptica no domínio do tempo utilizada para monitoramento opera em um comprimento de onda óptico separado e possui ampla largura de banda de detecção, tornando-se relativamente cara. A busca por uma alternativa de monitoramento de alta precisão e baixo custo é de suma importância para a oferta de transmissão com garantia de qualidade de serviço. Neste trabalho, o uso de multiplexação por sub-portadoras é explorado, incorporando uma técnica de monitoramento de varredura de tom de baixa freqüência na transmissão de dados. Um modelo matemático para a resposta em freqüência do enlace foi derivado e a localização e a intensidade de uma falha são estimadas através da comparação com a amplitude e a fase detectadas da luz retroespalhadaa medida em que é feita uma varredura de freqüência do sinal de monitoramento, aplicando-se um algoritmo de minimos quadrados. O modelo proposto considera que a medida do enlace antes da ocorrência de uma falha é conhecida. Uma única falha nova pode então ser detectada com precisão e sua posição e intensidade são estimadas da maneira descrita.

\section{Palavras-chave}

Monitoramento de Fibras Ópticas; Reflectometria Óptica no Domínio do Tempo; Reflectometria Óptica no Domínio da Frequência; Monitoramento de Fibras em Serviço; Multiplexação por Sub-portadoras; Redes Ópticas Passivas; Redes de Transporte 5G; Monitoramento de Camada Física 


\section{Table of contents}

1 Introduction $\quad 14$

2 Fiber Monitoring Techniques $\quad 17$

2.1 Optical Time Domain Reflectometry 18

2.2 Frequency Domain Reflectometry 21

2.3 Other Reflectometry Techniques 23

3 Passive Optical Networks $\quad 25$

3.1 Monitoring PONs 26

$\begin{array}{ll}3.2 \text { Challenges } & 30\end{array}$

3.3 SCM-PON 30

4 Proposed Solution $\quad 33$

4.1 Methodology 34

$\begin{array}{lll}4.2 \text { Setup } & 36\end{array}$

5 Mathematical Model $\quad 38$

5.1 Single Link Model 39

5.2 PON Model 43

6 Simulations $\quad 50$

6.1 Simulations using the Complete Mathematical Model 50

6.2 Simulations using the Differential Mathematical Model 62

$\begin{array}{llr}7 & \text { Experimental Results } & 69\end{array}$

$\begin{array}{lll}7.1 & \text { Coherent Rayleigh Noise } & 70\end{array}$

7.2 Experiments with Complete Mathematical Model for Estimation 73

7.3 Experiments with Differential Mathematical Model for Estimation 78

$\begin{array}{lll}8 & \text { Conclusions } & 87\end{array}$

$\begin{array}{llr}\text { A Differential Signal Model } & 98\end{array}$ 


\section{List of figures}

$\begin{array}{lll}\text { Figure 1.1 Summary of motivation for this study } & 15\end{array}$

$\begin{array}{lll}\text { Figure 2.1 Generic model for the optical reflectometer } & 18\end{array}$

$\begin{array}{lll}\text { Figure 2.2 } & \text { Block Diagram for the OTDR [1] }\end{array}$

Figure 2.3 Possible backscatter trace from a FUT [2] 20

Figure 2.4 Commercially available OTDR Equipment 21

Figure 2.5 Block diagram for the I-OFDR [3] 22

Figure 2.6 OFDR with coherent detection [1] 23

Figure 3.1 Block diagram for a Passive Optical Network 25

Figure 3.2 OTDR monitoring with reference reflector [4] 27

Figure 3.3 Tunable OTDR for PON monitoring [4] 27

Figure 3.4 Brilloin OTDR for PON monitoring [4] 28

Figure 3.5 SCM transmission in Radio Over Fiber [5] 31

Figure 4.1 Detected noisy signal based on mathematical model 33

Figure 4.2 Proposed scheme for the monitoring comparison process 35

$\begin{array}{lll}\text { Figure } 4.3 & \text { Experimental setup } & 37\end{array}$

Figure 5.1 Single fiber link of length $L$ and single fault at position $d \quad 40$

Figure 5.2 Single fiber link with multiple faults 42

Figure 5.3 PON with $N=2$ and splitter insertion loss $\delta_{s} \quad 44$

$\begin{array}{lll}\text { Figure 5.4 PON with } 2 \text { faults in separate branches } & 47\end{array}$

Figure 6.1 Long link simulation $(d=4100 m) \quad 51$

Figure 6.2 Long link simulation $(d=4100 m)$ - Polar plots $\quad 52$

Figure 6.3 Long link simulation $(d=8300 m) \quad 53$

Figure 6.4 Long link simulation $(d=8300 m)$ - Polar plots $\quad 54$

Figure 6.5 Short link simulation $(d=150 \mathrm{~m}) \quad 54$

Figure 6.6 Short link simulation $(d=150 m)$ - Polar plots $\quad 55$

Figure 6.7 Short link simulation $(\delta=3 d B) \quad 56$

Figure 6.8 Short link simulation $(\delta=3 d B)$ - Polar plots $\quad 57$

Figure 6.9 Short link simulation - Statistical analysis 58

Figure 6.10 Short link simulation - Estimation error comparison $\quad 59$

Figure 6.11 Short link simulation with known existing fault 60

Figure 6.12 1:4 PON Simulation 61

Figure 6.13 Long link simulation - Differential estimation 63

Figure 6.14 Short link simulation - Differential estimation 64

Figure 6.15 PON simulation (fault in feeder) - Differential estimation 65

Figure 6.16 PON simulation (fault in branch) - Differential estimation 67

$\begin{array}{lll}\text { Figure 7.1 } & \text { CRN Mitigation } & 72\end{array}$

$\begin{array}{lll}\text { Figure 7.2 } & \text { Modified experimental setup } & 72\end{array}$

$\begin{array}{lll}\text { Figure 7.3 KAP-10 laser characterization } & 73\end{array}$

Figure 7.4 Experimental setting for generic point-to-point link 74

Figure 7.5 Experimental results for $12 \mathrm{~km}$ single fiber link $\quad 75$ 
Figure 7.6 Polar plots for experiment with $12 \mathrm{~km}$ single fiber link $\quad 76$

Figure 7.7 Polar plots for experiment with $1.1 \mathrm{~km}$ single fiber link $\quad 77$

Figure 7.8 Experiment with $1.1 \mathrm{~km}$ single fiber link 77

Figure 7.9 Experimental result of estimation of very small faults 78

Figure 7.10 Mitsubishi laser characterization $\quad 79$

Figure 7.11 OTDR reference measurements $(d=850 \mathrm{~m}) \quad 80$

Figure 7.12 OTDR reference measurements $(d=2150 \mathrm{~m}) \quad 80$

Figure 7.13 Experimental results $(d=850 m) \quad 81$

Figure 7.14 Experimental results $(d=2150 \mathrm{~m}) \quad 82$

Figure 7.15 Experimental setting for generic PON link 82

Figure 7.16 OTDR measurements for PON setting (1). 83

Figure 7.17 OTDR measurements for PON setting (2). 84

Figure 7.18 Experimental results (PON) 85 


\section{List of tables}

Table 6.1 Point-to-point link simulation with Differential Estimation 63

Table 6.2 Simulation with Differential Estimation for various positions 64

Table 6.3 PON link simulation with Differential Estimation 66

Table 7.1 Measured and estimated faults for $1.1 \mathrm{~km}$ experiment 76

Table 7.2 Differential Estimation Result for PON mimicking setting 86 


\section{List of Abreviations}

SCM - Sub-Carrier Multiplexing

PON - Passive Optical Networks

OTDR - Optical Time Domain Reflectometry

QoS - Quality of Service

LMS - Least Mean Squared

OFDR - Optical Frequency Domain Reflectometry

RoF - Radio over Fiber

RBS - Rayleigh Backscattering

CW - Continuous Wave

FM - Frequency Modulation

AM - Amplitude Modulation

SNR - Signal to Noise Ratio

$\mathrm{PD}$ - Photodiode

ADC - Analog to Digital Converter

DSP - Digital Signal Processing

FUT - Fiber Under Test

IFFT - Inverse Fast Fourier Transform

LTI - Linear Time Invariant

VSA - Vector Spectrum Analyzer

FMCW - Frequency Modulation Continuous Wave

IF - Intermediate Frequency

DUT - Device Under Test

OWDR - Optical Wavelet Domain Reflectometry

$\mathrm{CO}$ - Central Office

RN - Remote Node

ONT - Optical Network Terminal

WDM - Wavelength Division Multiplexer

TDMA - Time Division Multiple Access

AWG - Arrayed Waveguide

CRN - Coherent Rayleigh Noise

LD - Laser Diode 
If we knew what it was we were doing, it would not be called research, would it?

Albert Einstein, 


\section{Introduction}

Advances in communications technology with more users and high level applications have led to an increase in bandwidth requirements from network providers. Fiber networks have proven to be a great source for high speed and high bandwidth communications to meet this need. Within this field, a subject of great importance is monitoring of the fiber characteristics which allows for maintaining the level of service provided. Monitoring schemes are expected to detect the occurrence of faults along an optical fiber link and to determine when and where a maintenance team must be deployed with minimum service disruption.

The choice of reflectometry as a monitoring method of preference is due to its ability to perform link analysis from a single end, which may be located at the service provider's facilities, thus avoiding disturbances to the end-user. Single-ended reflectometry technology in optical links is possible mainly due to a property of optical fibers called Rayleigh Backscattering [6-8] which will be discussed to the point of its importance to the matter of study.

Early research on fiber monitoring techniques led to the development of the Optical Time Domain Reflectometer (OTDR) [9] in the late 1970's. This technique is already widely used for monitoring the performance of optical fiber links and commercial OTDR equipment is available in the market. Advances in OTDR solutions and limitations to its employment in monitoring optical networks have been the subject of much research throughout the years and will be discussed further on in this study.

Another monitoring technique based on optical reflectometry that employs frequency sweeping instead of time averaging in a similar fashion to the OTDR is the Optical Frequency Domain Reflectometry (OFDR) originally presented in [10]. Although many studies investigate the use of OFDR and its advantages and disadvantages compared to the OTDR, this technique is not commonly employed to line monitoring.

In more recent developments, research efforts have been focused in embedding monitoring schemes into the transceiver of the optical communications system [11-13] in order to avoid service interruption during preventive maintenance. A cost effective solution with embedded monitoring that complies with 
network operators requirements for any kind of fiber link is not yet available.

Additionally, for fifth generation wireless applications, sub-carrier multiplexed $[14,15]$ radio over fiber (SCM-RoF) [16] is the likely method of choice for access networks data aggregation. It enables better utilization of the infrastructure of the network combined with the broad bandwidth of the optical fiber. Hence, the possibility for embedding a monitoring solution over one of the sub-carriers arises.

This study presents theoretical and empirical experimental results for performing a monitoring scheme using a sub-carrier channel in a SCM environment. The method of choice employs a similar methodology to an OFDR, with a frequency sweep of the amplitude modulation signal within the band of one sub-carrier. The analysis in this research actually employs a much shorter frequency range limited to a few hundred $\mathrm{kHz}$. The novelty of the proposed solution resides mainly in providing a monitoring signal that can be embedded in the SCM-RoF transmission without interfering in data transmission, assuming a separate sub-carrier block is reserved for monitoring (possibly shared with other signaling functions like synchronization). Figure 1 presents a summary of the motivation for pursuing this work.

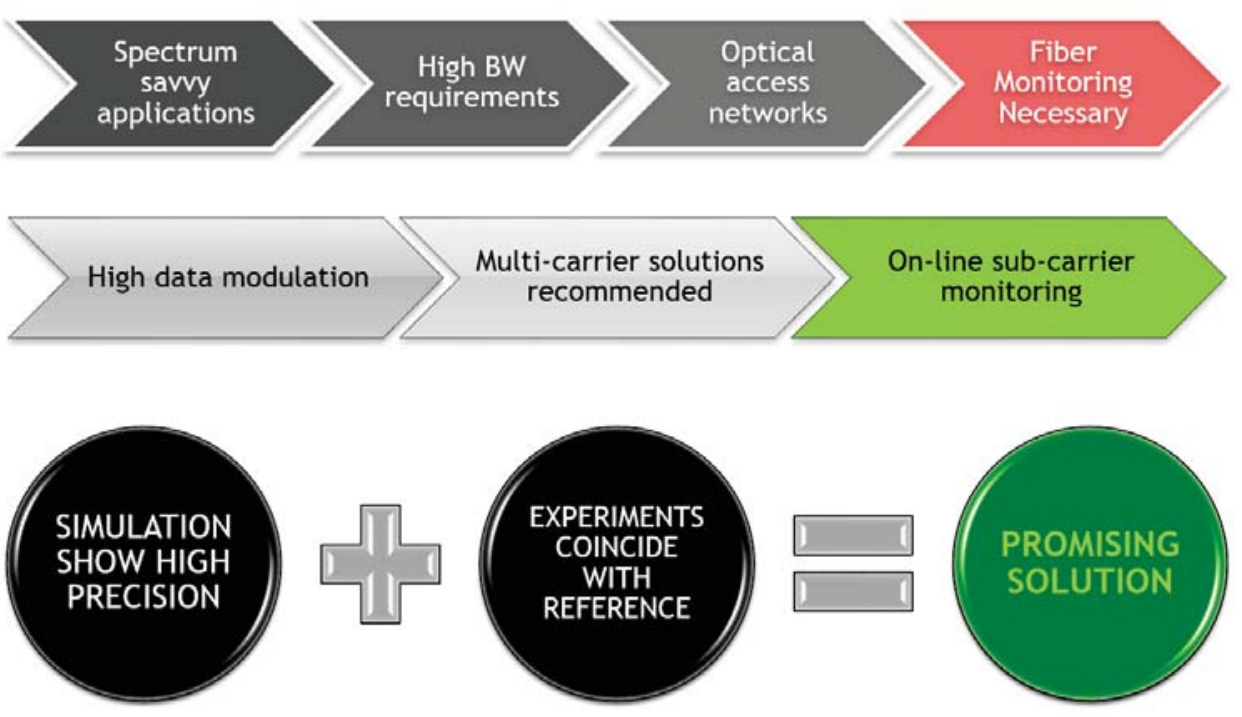

Figure 1.1: Summary of motivation for this study

Another important aspect under investigation here is the possibility of extending the proposed scheme for monitoring passive optical networks (PONs). So far, none of the available monitoring methods can detect faults in branched networks in a cost effective timely manner. Following the proposed methodology, a fault location, loss factor intensity and corresponding network 
branch can be detected with somewhat reasonable precision. This solution is an original approach for online monitoring of PONs.

In chapter 2 , the existing monitoring techniques are summarily presented. More detailed information about them can be found in the references given throughout this work. Chapter 3 describes branched optical networks in relation to the scope of this work and introduces relevant aspects of monitoring them. Some details about the challenges posed in monitoring PONs are discussed and possible ways to overcome them are presented. The proposed solution is introduced in chapter 4 . The theoretical analysis and mathematical modeling developed are discussed in chapter 5 , followed by simulation results in chapter 6. Empirical experiments for monitoring both single links and branched optical networks are included in chapter 7. A short conclusion is given in chapter 8. An expansion of the mathematical derivation for the model used in this thesis has been added in Appendix A.

Computer aided simulations indicate that the method provides accurate results in finding a new fault occurrence and estimating its intensity for both point-to-point links (single link) and point-to-multi-point branched networks (PONs). The experimental results validate the insight given by the simulations and show results comparable to measurements performed with a commercial OTDR used for reference. The advantage of the proposed method relies on that it is embedded in the data signal, occupying the baseband sub-carrier in the SCM transmission, and doesn't require additional costly equipment (especially at the user end). This solution is suitable for monitoring Radio over Fiber (analog RoF) transmission with sub-carrier multiplexed electrical channels transmitted through a fiber access network. 


\section{2}

\section{Fiber Monitoring Techniques}

In communications it is very important to characterize and constantly monitor the network in order to guarantee the contracted service provision level. For fiber optical based networks monitoring is performed through optical reflectometry techniques. In this chapter some of the solutions for fiber optics monitoring existing commercially and in current literature are presented and discussed.

Optical reflectometry is basically described by a set of techniques where a signal is sent through an optical device or network and the reflected and backscattered signal received at the input end (usually using an optical circulator) is analyzed to infer the device or network properties. Measurements of the intensity of the light signal detected in such fashion can be related to information about loss events such as splices, connectors and breaks, as well as about the characterization (attenuation distribution, refraction index, length,...) of the fiber or device. It is a very important tool in characterizing optical components as well as in the detection of faults and failures. Reflectometry techniques are specially interesting because they generally only need to access a single end of the fiber (both source and detector are collocated). They rely on an intrinsic characteristic of the fiber called Rayleigh Backscattering (RBS).

Rayleigh scattering is a phenomenon which occurs due to microscopic imperfections in the fibers which are much smaller than the wavelength of the transmitted light. These imperfections are present in a random fashion throughout the length of the fiber and cause scattering of a portion of the transmitted light where they occur. Some of this light is recaptured and back reflected towards the input of the fiber. Optical reflectometry techniques measure the intensity of this light (related to distributed reflections format of RBS) along with light coming from punctual reflections due to purely reflective elements.

Generally, in optical reflectometry techniques a test optical signal that may be continuous wave (CW), chirped, pulsed, frequency modulated (FM) or amplitude modulated (AM) is inserted at the input to the fiber, optical device or network. The returning signal at the same wavelength, which results from 
a combination of localized reflections and Rayleigh Backscattering, is detected at the same end of the fiber, usually through a circulator, and analyzed.

The backscattered signal detected by an optical reflectometer presents a time delay or frequency variation that can be related to the position of the reflective elements within the device under test. The speed of light in the fiber (group speed) $v_{g}=\frac{c}{n_{g}}$ may be used to infer the position of the reflection (distance from the input) from the delay measured. Signal processing is used to determine the magnitude of each reflective element and its corresponding location. Figure 2.1 shows a block diagram representing the generic model for the optical reflectometer techniques.

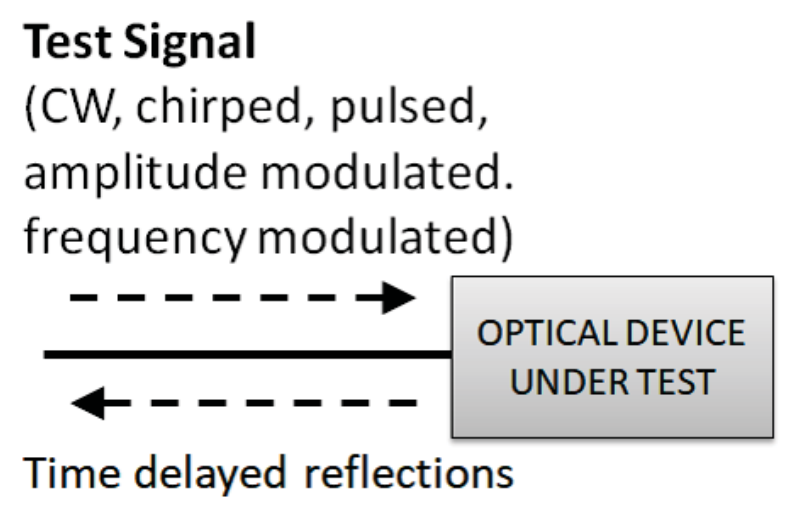

Figure 2.1: Generic model for the optical reflectometer

Currently employed reflectometry methods are described thoroughly in [3]. More detailed information on Rayleigh Backscattering in optical fibers (or devices) is found in [6-8,17-19]. Some general concepts about the most common optical reflectometry techniques are discussed next.

\section{1}

\section{Optical Time Domain Reflectometry}

The most widespread optical reflectometry technique nowadays consists of sending an optical pulse of short duration through an optical circulator into the device or fiber under test and measuring the intensity and delay of the backscattered signal arriving from it (in the opposite direction from the transmission) over time. In practice, a train of pulses with sufficiently long intervals between pulses is sent and the received signal averaged over its period in order to improve the measurement's signal-to-noise ratio (SNR). Since the method employs short optical pulses in time it is referred to as Optical Time Domain Reflectometry, or by its acronym OTDR.

Early measurements related to the OTDR technique are found in $[9,20]$. A thorough description of the OTDR technique and the related mathematical 
formulation are described in a summarized manner in $[1,21]$. More information about this technique and related research can be found in $[22,23]$.

A block diagram of an OTDR with direct detection, also known as incoherent OTDR or simply I-OTDR, is presented in figure 2.2. The laser diode is driven by the electrical pulse generator producing an optical pulse train that is input into the fiber or device under test. A photodiode (PD) is used to detect the backscattered signal arriving back at the input end of the device, through a directional coupler or circulator. The detected signal is amplified and then digitalized by the analog-to-digital converter (ADC). Next, it is analyzed by the digital signal processing (DSP) unit that calculates the relative delay of the pulse in each section of the fiber (or device) generating a trace from which its characteristics may be inferred. The DSP unit must be synchronized with the pulse generator (the source) for the delays to be computed correctly.

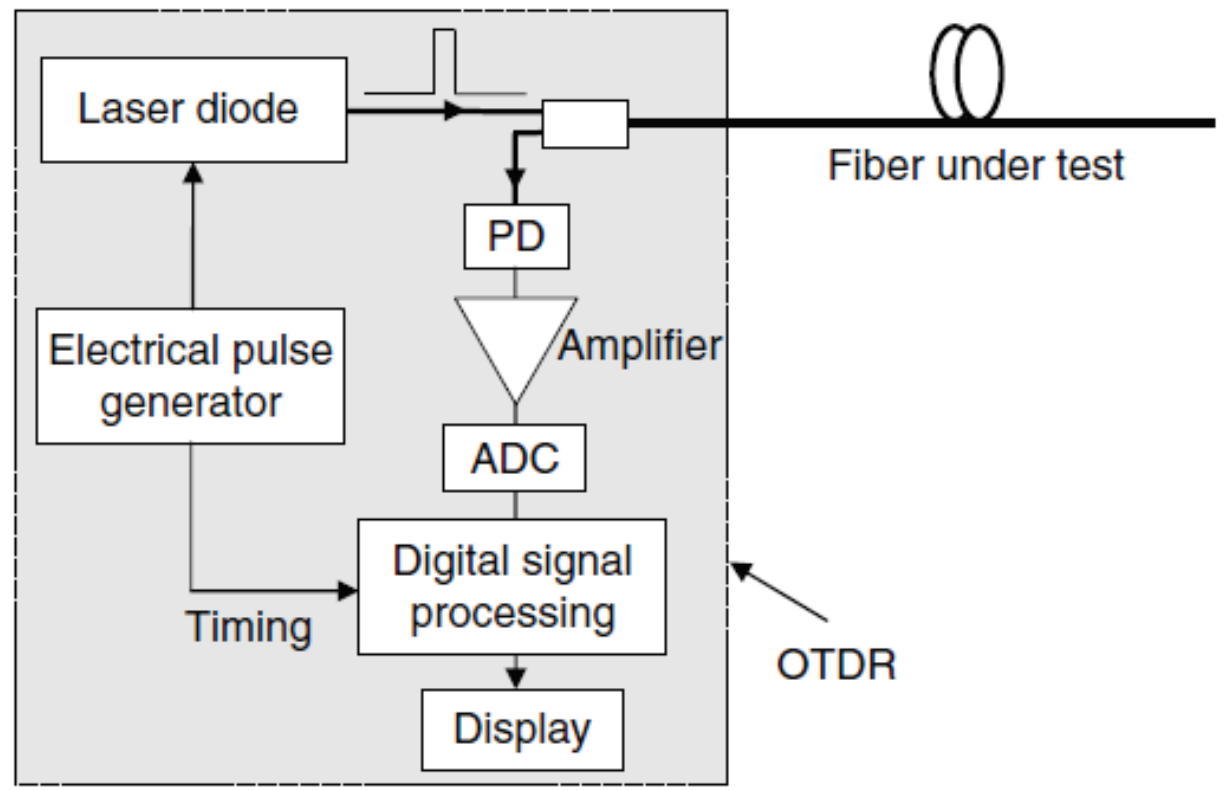

Figure 2.2: Block Diagram for the OTDR [1]

The time/delay dependence of the detected signal is given by the relative distance from the origin by $2 \Delta z=v_{g} \Delta t$. This relation is useful to determine the position of events (bend, break, end of fiber, ...) along the length of a fiber. Figure 2.3 depicts an example of a possible OTDR trace for a fiber under test (FUT).

The DSP unit is also responsible for averaging over the signals coming from the subsequent pulses in order to reduce the effect of thermal noise and dark current noise caused by the detection scheme. I-OTDR sensibility is limited by thermal noise in the reception and the dark current in the 


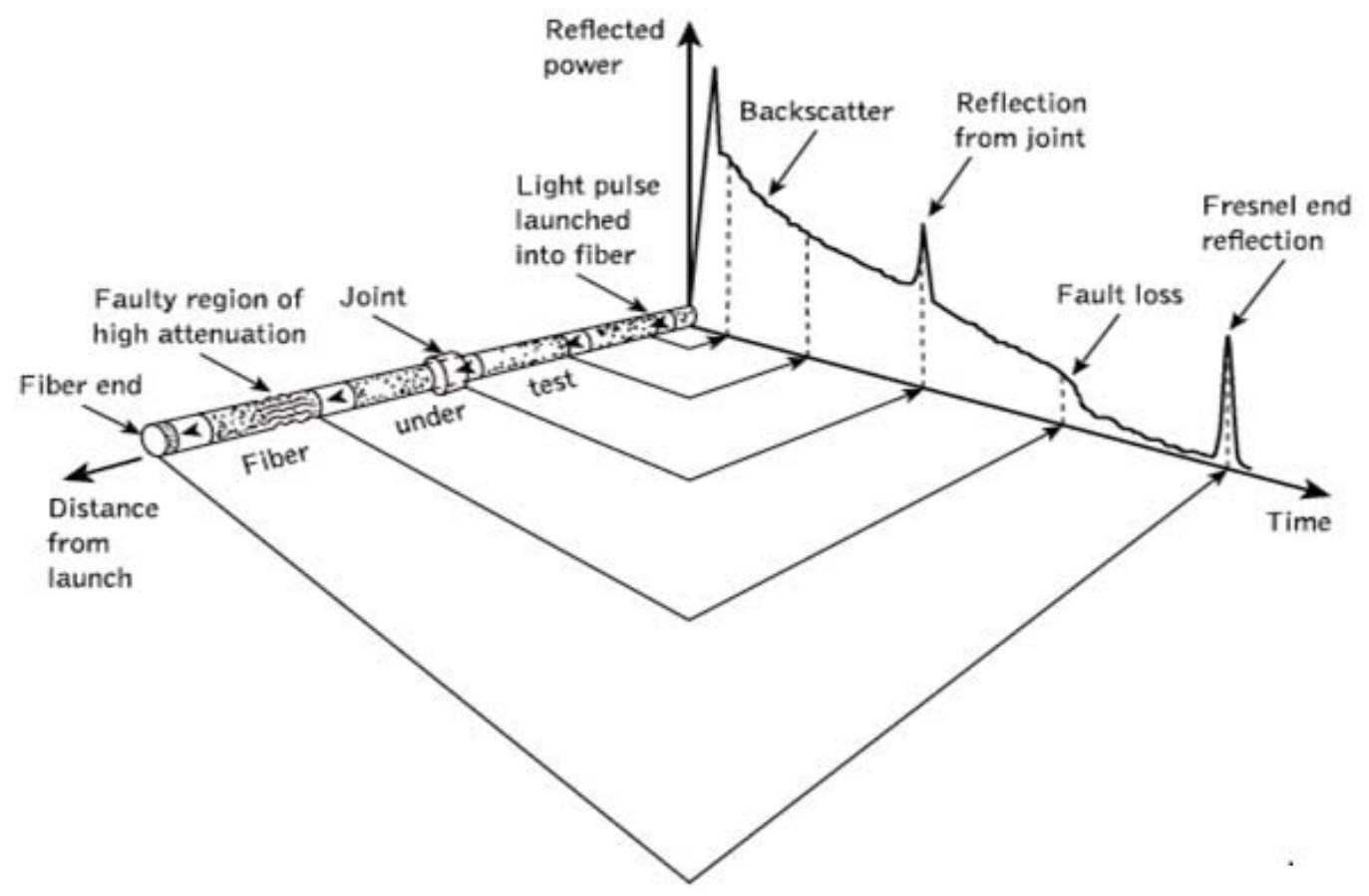

Figure 2.3: Possible backscatter trace from a FUT [2]

photodiode. Many solutions have been proposed to improve results on this matter including coherent detection, coding, photon counting among other optical reflectometry techniques. More information on them may be found in $[24-34]$.

One important factor to determine the efficiency of the measurement method is spacial resolution, that is the ability to differentiate between two events close together. For an OTDR, the spacial resolution is generally dependent on the pulse duration $\tau_{p}$ according to equation 2.1. For better resolution (smaller $\Delta z_{r}$ ), the input pulse must be narrower. This factor is usually limited by proprieties of the light source and its modulating equipment (laser diode and pulse generator). Alternative reflectometry techniques can be employed in an attempt to overcome this limitation.

$$
\Delta z_{r}=\frac{1}{2} v_{g} \tau_{p}
$$

As previously mentioned, the OTDR is a very widespread and well established method to determine magnitude and locations of faults and reflections as well as propagation characteristics of the fiber in point-to-point optical fiber networks. OTDR equipment is broadly available commercially in the market from various vendors (an example is presented in figure 2.4). It is commonly used by optical communications service providers for fiber network installation, 
monitoring and maintenance.

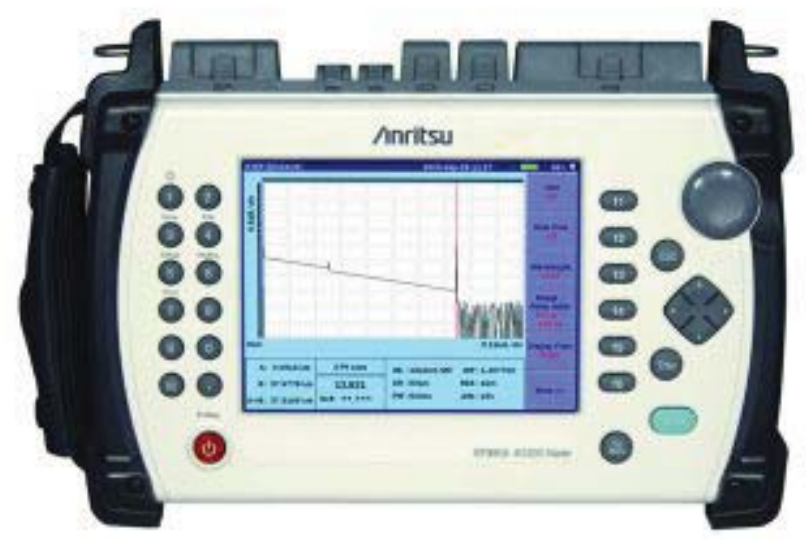

Figure 2.4: Commercially available OTDR Equipment

\section{2}

\section{Frequency Domain Reflectometry}

A clear alternative to the approach of time-domain reflectometry is to employ a measurement technique in the frequency-domain. In this solution, instead of a short pulse, the laser diode emits a continuous wave signal with varying frequency. The detected signal is obtained as a function of the corresponding input frequency and the Inverse Fourier Transform can be performed to obtain the trace as a function of time delay, and subsequently of distance. Usually the fast version of the transform called inverse Fast Fourier Transform (IFFT) is used. This technique conveniently called Optical Frequency Domain Reflectometry (OFDR) is also known as Optical Fourier Domain Reflectometry in a clear reference to the employment of the Fourier Transform.

This approach is possible because the Rayleigh Backscattering is a linear process and the typical device under test can be considered to behave like a linear time invariant (LTI) system. Therefore, measuring its impulse response is equivalent to evaluating the transfer function magnitude and phase as a function of the input frequency and calculating the IFFT. A review of the variations of OFDR techniques is presented in [35] and briefly described next.

The basic concepts employed in an OFDR are illustrated in figure 2.5: a sinusoidal test signal is generated with a vector spectrum analyzer (VSA), or with separate equipment that is also used to generate the reference signal to the VSA, and launched into the fiber or device under test. The transfer function is obtained by measuring the magnitude and phase of the reflected 
signal detected by a high frequency (wide bandwidth) detector, for varying frequencies of the sinusoidal test signal. Some sort of frequency sweep method is generally employed to vary the frequency of the input signal. The IFFT of the measurement corresponds to the reflectometer trace. Since this technique is analog to the I-OTDR previously described, it is often referred to as Incoherent OFDR (I-OFDR).

In a similar fashion to the OTDR, the OFDR trace acquired from the IFFT is related to the distance the light traveled in the fiber or device according to: $\Delta z=\frac{v_{g}}{2} \frac{1}{\Delta f_{s}}$, where $\Delta f_{s}=f_{\max }-f_{\min }$ represents the frequency span of the measurement.

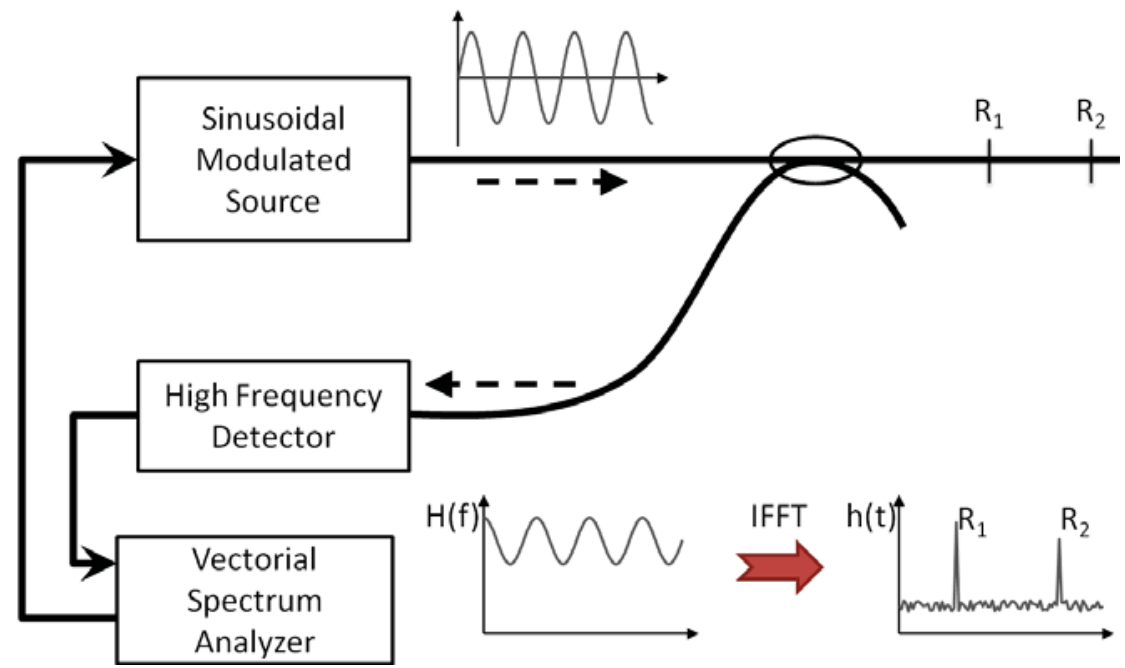

Figure 2.5: Block diagram for the I-OFDR [3]

A variation of the I-OFDR known as Frequency Modulation Continuous Wave (FMCW) produces similar results without the need for a spectrum analyzer. The reflected signal is mixed with the input signal at the same modulation frequency generating intermediate frequency (IF) beat signals at frequencies that are then related to the delay of the corresponding reflection and consequently to its position.

Some experiments with I-OFDR are presented in $[10,36-40]$. In most cases punctual reflections are easily detected, measured and located, but distributed loss measurements from Rayleigh Backscatter were often disregarded. With increased bandwidth requirements and more complex modulation schemes being used, monitoring of the distributed loss has grown in importance and should no longer be dismissed.

As mentioned before for the OTDR, coherent detection techniques have also been studied with OFDR to improve sensibility. With coherent detection it is possible to measure both intensity and phase of the backscattered signal allowing for very high resolution measurements. Nonetheless, this technique is 
highly sensitive to the stability of the medium as small variations may cause great distortion in the measurements. Another disadvantage of this method is the requirement for a high coherence laser, implicating in greater phase noise which reduces the dynamic range, thus events that are close together cannot be set apart.

Figure 2.6 shows a variation of OFDR with coherent detection. The interference signal between the reference arm with a fixed optical mirror at a known distance and the device under test (DUT) is used to determine the position of reflective events using Fourier domain analysis. In this simplified solution, distributed loss cannot be determined. Studies involving coherent OFDR (C-OFDR) techniques are presented in [41-46]. Although this technique clearly offers better measurements, it may be much more expensive and hard to implement due to the high coherence laser and stability requirements.

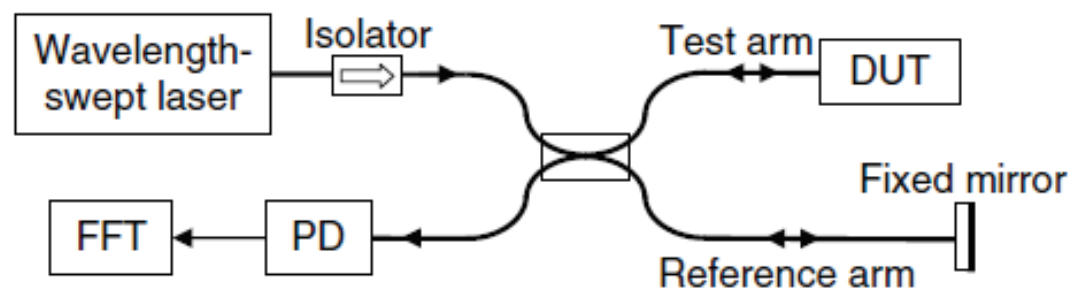

Figure 2.6: OFDR with coherent detection [1]

Commercial equipment that perform OFDR measurements are not currently widely available as it is the case of OTDR measurement equipment. If such a scheme is required, the network administrator usually acquires a custom made solution.

\section{3}

\section{Other Reflectometry Techniques}

Alternate optical reflectometry techniques are, in a general sense, variations and improvements on the OTDR and OFDR solutions. Some of these techniques have already been mentioned such as correlation or coding OTDR $[25,27,28,30]$ and OTDR with photon counting [32,33].

Other approaches studied include polarization control [47] and phase measurements $[48,49]$ or both [50] to acquire an accurate reflectometry trace of the device under test. Some study has also been dedicated to other domain solutions such as Optical Wavelet Domain Reflectometry (OWDR) [51].

None of these methods alone offer a fully efficient, timely solution for the service providers' needs in monitoring their networks without service disruption. Much focus has been given to research with embedded OTDR solutions 
including [52-56]. Other recent studies have been focused on embedding the monitoring applications within the data transmission system itself [11-13]. An alternative to the solutions described is provided in this work. 


\section{3}

\section{Passive Optical Networks}

Passive optical networks (PONs) are a cost effective choice for local access networks where costly equipment can be shared by multiple subscribers, offering high capacity telecommunications solutions. In order to ensure reliability of services over such networks, it is highly important to implement a good monitoring technique. Furthermore, since the shared equipment is located at the central office $(\mathrm{CO})$ it is desirable that the monitoring solution be implemented in a remote manner from this location.

The block diagram of a generic PON as it will be considered throughout this work is presented in figure 3.1. The active equipment is located at the CO and connected to the passive splitter at a remote node $(\mathrm{RN})$ by a fiber section known as a feeder fiber, referred to also as feeder line or simply feeder. Each branch in the network connects the RN to the optical network terminal (ONT) at each of the end-users' facilities. The splitter in the RN is usually either a wavelength division multiplexer (WDM) or an optical power splitter.

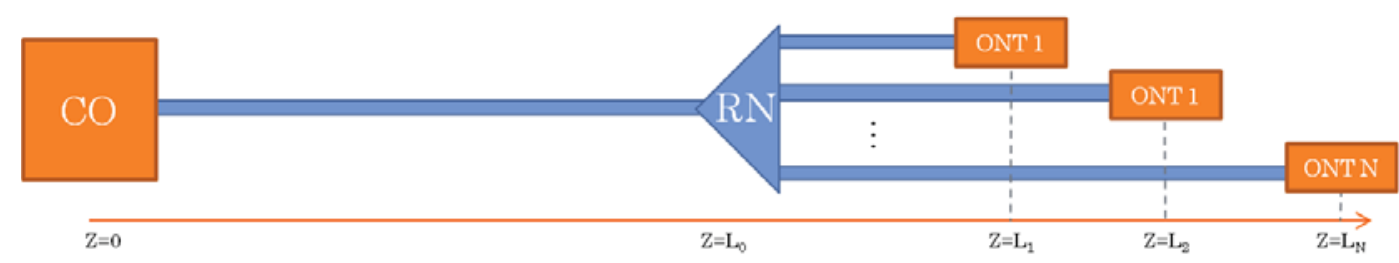

Figure 3.1: Block diagram for a Passive Optical Network

In this network structure it is a common requirement that all active equipment, including the network monitoring functions be concentrated at the central office. However, monitoring a drop fiber section or branch from the CO is a complex problem because the reflectometry results measured are derived from an overlap (sum) of the measurements of all the multiple drop fiber branches.

Measuring a fault within the overlap and identifying in which branch it is located in presents one of the challenges in PON monitoring. Another issue is the case where multiple faults occur at the same distance from the measuring device and the contribution from each one must be separated from the rest. Furthermore, for the simpler passive optical networks which employ 
power splitters at the RN and Time Division Multiple Access (TDMA), also known as TDM-PONs, the passive splitter introduces a high loss, of the order of the number $N$ of branches, which is usually interpreted as a fiber break by most of the existing reflectometry techniques.

A straightforward solution would be to implement monitoring at each branch from the ONTs on the end-user side $[57,58]$ and send the measured results to the $\mathrm{CO}$ along with upstream communications, but it would be simpler and more cost effective to monitor all users directly from the CO. This work is focused on solutions for centralized monitoring only.

Multiple approaches to this problem have been researched and some of them will be presented and discussed next. This research attempts to provide an alternative solution that may be implemented at the CO in PON networks employing mostly the same equipment used in downstream data transmission, thus at a reduced monitoring cost.

\section{1}

\section{Monitoring PONs}

Despite the claims of manufacturers, current reflectometry equipment are not efficient in monitoring branched networks. Most solutions employed at present rely on some kind of two-step analysis, where the distance and intensity of a fault are estimated in one step and the branch in which it is located is determined in a separate step (either prior to estimating the exact location and intensity of the fault or through some processing to isolate the branch information from the overlapped reflectometry trace). Some of these proposed solutions are summarized in [4]. Some of the solutions discussed are shown in figures 3.2, 3.3 and 3.4, corresponding to monitoring with a reference reflection at the user end, using different wavelengths for monitoring each user and using special fibers with unique Brillouin frequency shift for each ONU, respectively.

The method subject of this study offers the possibility to try and overcome the challenges posed in monitoring a branched passive optical network described earlier. For a TDM-PON, a mathematical model has been developed so that an estimate of the location and loss intensity of a fault, including the corresponding network branch, can be found using a data fitting algorithm in a similar fashion to the point-to-point solution.

A WDM-PON is considered a special case of a point-to-point link from the point of view of the data communications wavelength. The arrayedwaveguide-grating (AWG) filter that separates the wavelengths for individual delivery is viewed as an existing fault at a known location $L_{0}$ corresponding to the feeder fiber length with loss intensity given by its insertion loss. A 


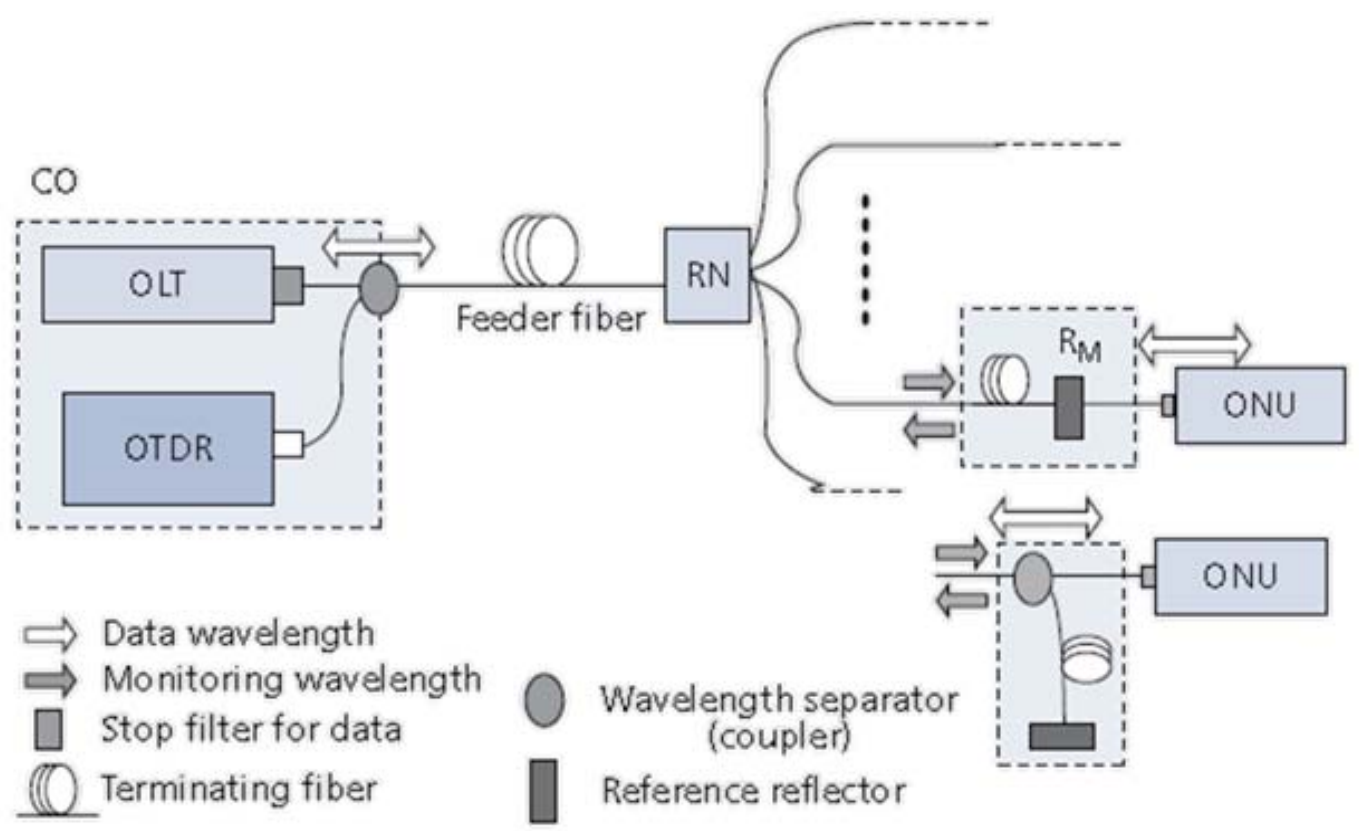

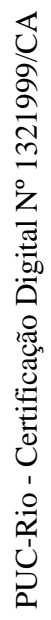

Figure 3.2: Use of a reference reflector for OTDR-based automatic monitoring of PONs [4]

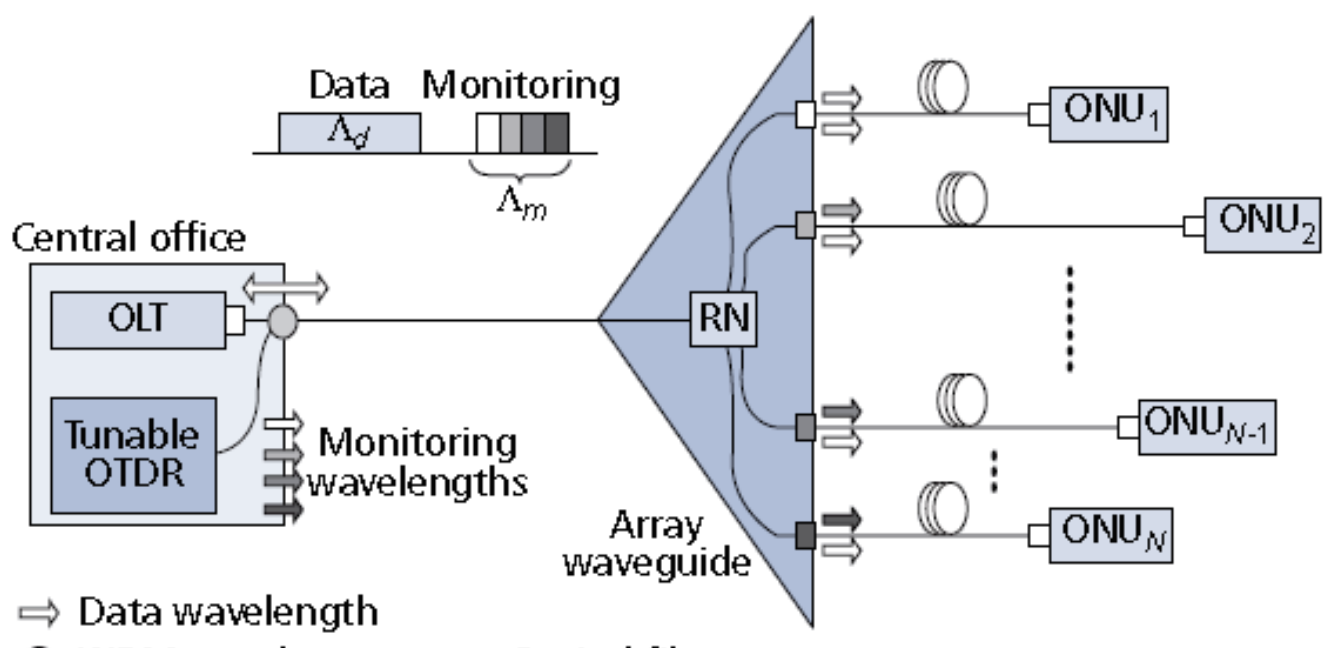

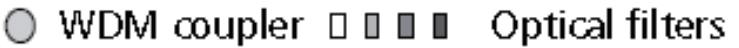

Figure 3.3: OTDR for PON via one monitoring wavelength per ONU [4] 


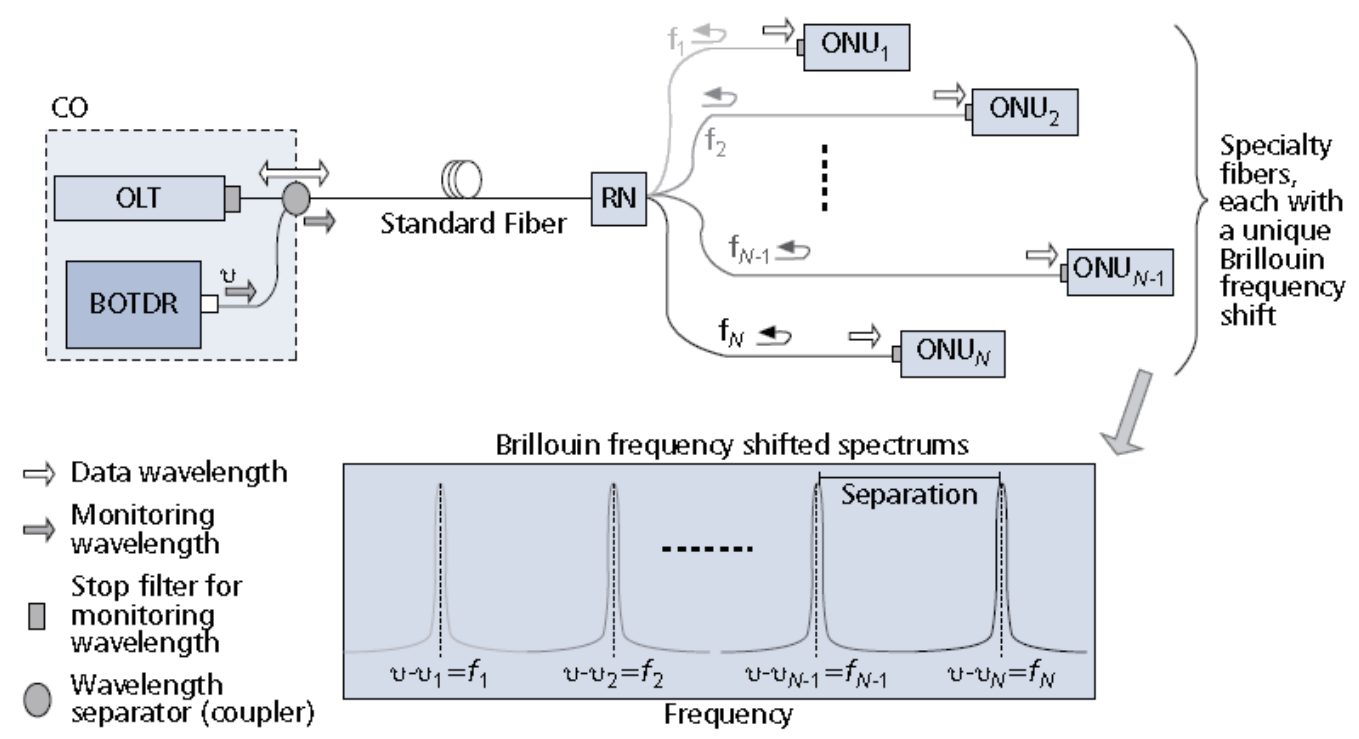

Figure 3.4: Performance monitoring based on Brillouin frequency shift assignment [4]

monitoring solution based on SCM would be necessarily employed separately for each wavelength $\lambda$ of the WDM-PON. This scenario will not be discussed any further here.

The mathematical model employed for the solution proposed in this work, in the TDM-PON case, will be described by the experimental setup presented and discussed in Chapter 4, where the fiber under test (FUT) is replaced by a feeder fiber of fixed length followed by set of parallel fiber links connected to it through an optical power splitter, representing a PON.

It is important to note that a commercial OTDR cannot determine precisely in which branch of a PON a fault has occurred without relying on additional measurements. In a controlled laboratory experiment where a fault is induced in a known branch, this information is available and a good solution should estimate which branch that is. Furthermore, the fault intensity measured by the OTDR represents an apparent loss which is masked within the sum of the backscattered light from all the branches and the actual loss factor must be calculated from it.

As mentioned before, there are a great number of studies suggesting different procedures to provide centralized monitoring of fiber branches in a PON structure. A concise review of solutions that have been studied may be found in [59].

Although early studies on monitoring passive optical networks date back to the 1990's [60-63], the subject is still relevant because a simple low cost effective solution is not yet universaly available. Recent studies on PON 
monitoring can be found in [64-67]. Most centralized monitoring strategies for PONs are based on employing a commercial OTDR or a variation of this equipment such as a wavelength tunable OTDR or some embedded OTDRlike methodology. They also often apply some kind of multi-step solution monitoring the branches one at a time, for example.

One simple solution suggests using the OTDR monitoring signal bypassing the optical splitter with different aproaches to achieve this outcome described in [68]. Generally, this solution is implemented by means of an active device $[69,70]$ or by some passive solution such as using a wavelength multiplexer/de-multiplexer as was suggested in [71,72].

The first option is only justifiable when considering installed networks, where the remote node is often already powered due to existing equipment from legacy network, or when the PON splitter is located within the $\mathrm{CO}$ and powering an active by-pass equipment is not an issue. The latter proposal considers that all active equipment is supposedly concentrated in the $\mathrm{CO}$, as is the case for a purely passive network, but its downfall is the requirement for expensive tunable OTDR equipment. Either solution aims to monitor each of the branches in the PON individually which may also lead to scalability limitations.

A variety of solutions exploiting the fact that OTDRs usually opperate at a separate wavelength for monitoring and adding reflective elements at the far end of fiber branches have been proposed in [73-76] among other.

An alternative approach studied in $[65,77,78]$ suggests evaluating the PON network as a whole and then employing additional means to separate the contribution of each branch to the combined OTDR measurement. Mostly, these solutions include the comparison with a prior knowledge of the network trace measured after installation and possibly updated as additional losses or changes in the network may occur.

Other solutions for the monitoring of PONs have been presented in [79-81] and more. These studies however do not present a complete and simple solution for localization and fault intensity estimation.

The goal of this work is to develop a simple and cheap solution for estimating fault location and corresponding loss intensity that can be embedded to the data signal and applied to monitoring PONs with equivalent accuracy compared to OTDR based solutions. Some of the challenges in the field of PON monitoring are presented next. 


\section{2}

\section{Challenges}

As previously mentioned, a reliable and inexpensive solution for the monitoring of branched passive optical networks is not yet widely available and is, therefore, subject of great research interest still. The main requirements for a monitoring method for PONs are summarized in [4], including difficulties to evaluate small faults that are masked by power from longer branches, determining the branch where a fault occurs and overcoming the huge loss factor detected at the splitter in the remote node.

It is important to note that detecting and localizing faults for PONs is more critical than for point-to-point links because of the high loss factor induced by the passive splitter which makes the transmission system more sensitive to imperfections in the point-to-multipoint case. For any method to work with PON monitoring, it needs to maintain its signal-to-noise level above the requirement after the power splitter.

Clearly, a centralized monitoring method employing optical reflectometry is a promising solution for branched passive optical networks. Most research in the field is focused on OTDR variations or similar reflectometry techniques, which is likely the best direction to achieve efficient one-sided fiber monitoring results.

The preferred mechanism should take into account that Rayleigh backscattered light from the different branches in the network overlap, possibly masking small failures, specially as the split ratio increases. Also, it is desirable that the proposed solution monitor the network in a single step and without the need for adding expensive elements. Furthermore, the scalability issue in terms of increasing the splitting ration and thus number of users sharing the infrastructure is critical for PON cost effectiveness and must be considered.

\section{3}

\section{SCM-PON}

In this section, a brief description of the sub-carrier multiplexing (SCM) solution will be offered. This scheme has been studied as a likely choice for multiplexing various users in 5th generation mobile communication networks $(5 \mathrm{G})$. Within this context, these networks are most likely going to rely on optical fiber for the access network, which can support large bandwidth (of the order of $100 \mathrm{M} \mathrm{Hz}$ combined data channels or more) and provide high data rates. Such transmission of the mobile radio communications data through the fiber to the radio heads is called Radio over Fiber (RoF). It will provide a 
reliable communication format for $5 \mathrm{G}$ access networks especially due to the fiber relative immunity to electromagnetic interference.

Sub-carrier multiplexing is a modulating scheme of obtaining multiple electrical channels through a combination of a set of bandpass signals with different central frequencies. It has been recommended for implementation of analog RoF solutions [5]. Figure 3.5 shows the block diagram representing SCM transmission for a RoF transmission. The electrical signal modulating the optical carrier $f_{\text {opt }}$ is composed of multiple frequency multiplexed channels.

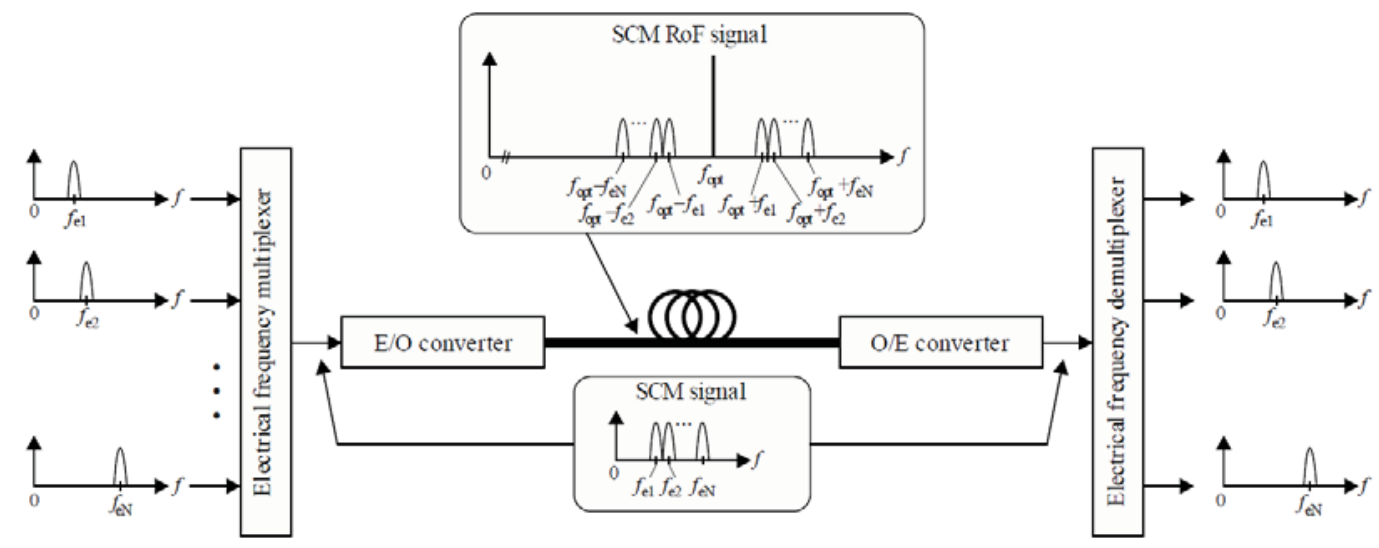

Figure 3.5: SCM transmission in Radio Over Fiber [5]

One limitation of SCM transmission is that the total optically modulated power is limited to avoid clipping effects and other distortions in the laser. The bias current of the laser should be such that it operates midway between the current threshold and through it's linear operating region in order to achieve maximal modulation depth without incurring non-linear effects. This restriction determines the maximal allowed power for each electrical sub-carrier. Assuming the total bias current excursion possible under these circumstances as $\Delta i_{\text {bias }}$ and a number $N_{p}$ of sub-carriers, then each electrical channel should contribute about $\frac{\Delta i_{\text {bias }}}{2 N_{p}}$ to the swing in the current modulating the laser (in each direction above and below the bias) because the SCM signals are sinusoidal.

In the context of branched optical networks (like PONs), this limitation poses a challenge because the splitter often has high insertion loss (growing exponentially with the splitting ratio). In fact the received power at the end of each branch is inversely proportional to the number of branches $N$. A monitoring solution that works with SCM-PONs using one sub-carrier for monitoring such as what is proposed in this work should have enough power in each sub-carrier to overcome the PON splitter loss, while restraining its power to a maximum of $\frac{1}{N_{p}}$ of the total modulation power allowed for operation 
within the laser linear region. This trade-off must be taken into account when implementing the proposed solution in a SCM-PON transmission setting. 


\section{4 \\ Proposed Solution}

The purpose of this work is to evaluate a novel approach to optical reflectometry taking advantage of sub-carrier multiplexing (SCM) to include a monitoring solution embedded in the data signal that provides fault localization in point to point links as well as point to multi-point passive optical networks.

The proposed solution may be implemented with the same equipment already used for data transmission with few additions by allocating one of the channels in SCM for monitoring, possibly along with other signaling and synchronizing schemes. This allows for a cheap online monitoring solution application. The low frequency (base band) sub-carrier was preferred because the mathematical model of the backscattered light signal suggests that signal to noise ratio deteriorates with the increasing modulating frequency. This happens because most noise sources affecting the system (thermal noise, shot noise, ASE,...) are represented by white noise, while the backscattered light intensity decays with the increase in the tone modulating frequency. Figure 4.1 presents that insight.

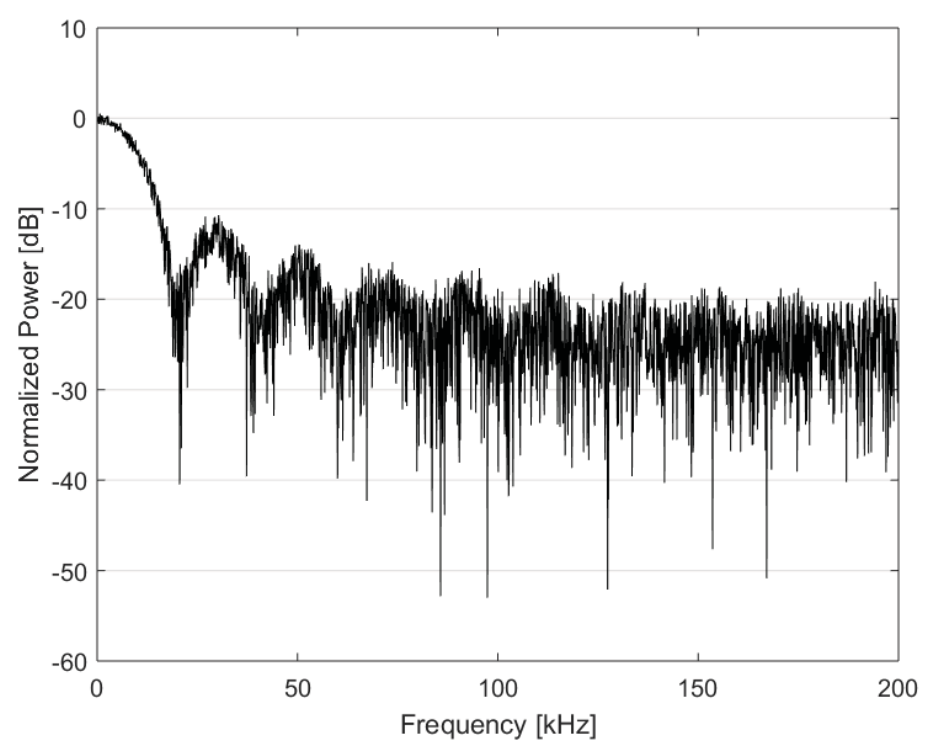

Figure 4.1: Detected Rayleigh backscattered signal, as a function of the input monitoring tone modulation frequency (simulated) 
Multiple fiber measurements were executed to validate the mathematical model which will be described further on in the next chapter. The related acquired data will be presented and discussed in the experimental results chapter.

In the suggested methodology employed for fiber monitoring, a mathematical model was determined for the behavior of the signal detected at the input to the fiber (or fiber network) when the low frequency sub-carrier is modulated by a frequency swept sine tone, under continuous-wave (CW) operation of the laser. The signal measured under these conditions is then compared to the expected model in order to predict the location and intensity of a fault occurrence in the fiber under test.

The mathematical formulation derived to describe the fiber links studied is somewhat complex leading to possible difficulties in curve fitting optimization, as the Least Squares Algorithm selected may not allways converge. As an alternative, a model for the change in the signal detected is proposed and may be used for comparison to the differential signal obtained by subtracting a new measurement with reference data evaluated under prior known circumstances corresponding to a simpler mathematical description.

The main novelty in this solution is the use of one of the SCM data transmission sub-carriers for monitoring through a low frequency optical reflectometry technique. Some primary results have been published in $[82,83]$ and a patent [84] was registered for this method. The goal of this work is to investigate the accuracy of using this Sub-Carrier Optical Frequency Domain Reflectometry (SC-OFDR) for point to point fiber links as well as its application for monitoring branched passive optical networks (PONs).

\section{1}

\section{Methodology}

In the research described in this work, fault location and intensity measurements are performed by introducing a frequency swept base band tone signal into the transmitting end of the fiber being tested, which can be combined with the data communications in a SCM setting where the base band sub-carrier is spared for signaling and control without disrupting the data transmission. The fault measurements are estimated from the returning signal resulting from Rayleigh backscatter (RBS) detected at the same end of the fiber through an optical circulator, in an equivalent manner to that of existing reflectometry techniques described in Chapter 2.

As mentioned, the backscattered signal detected with the proposed method is compared to the mathematical model of the expected signal response 
developed in the scope of this study, which will be presented further on, in order to estimate location and intensity of a fault occurrence employing optimization techniques based on fitting data to a curve modeled. Both amplitude and phase of the detected measurement signal were used and complex data fitting to a complex model was performed. A scheme describing the suggested monitoring process using the solution studied in this work is given in figure 4.2.

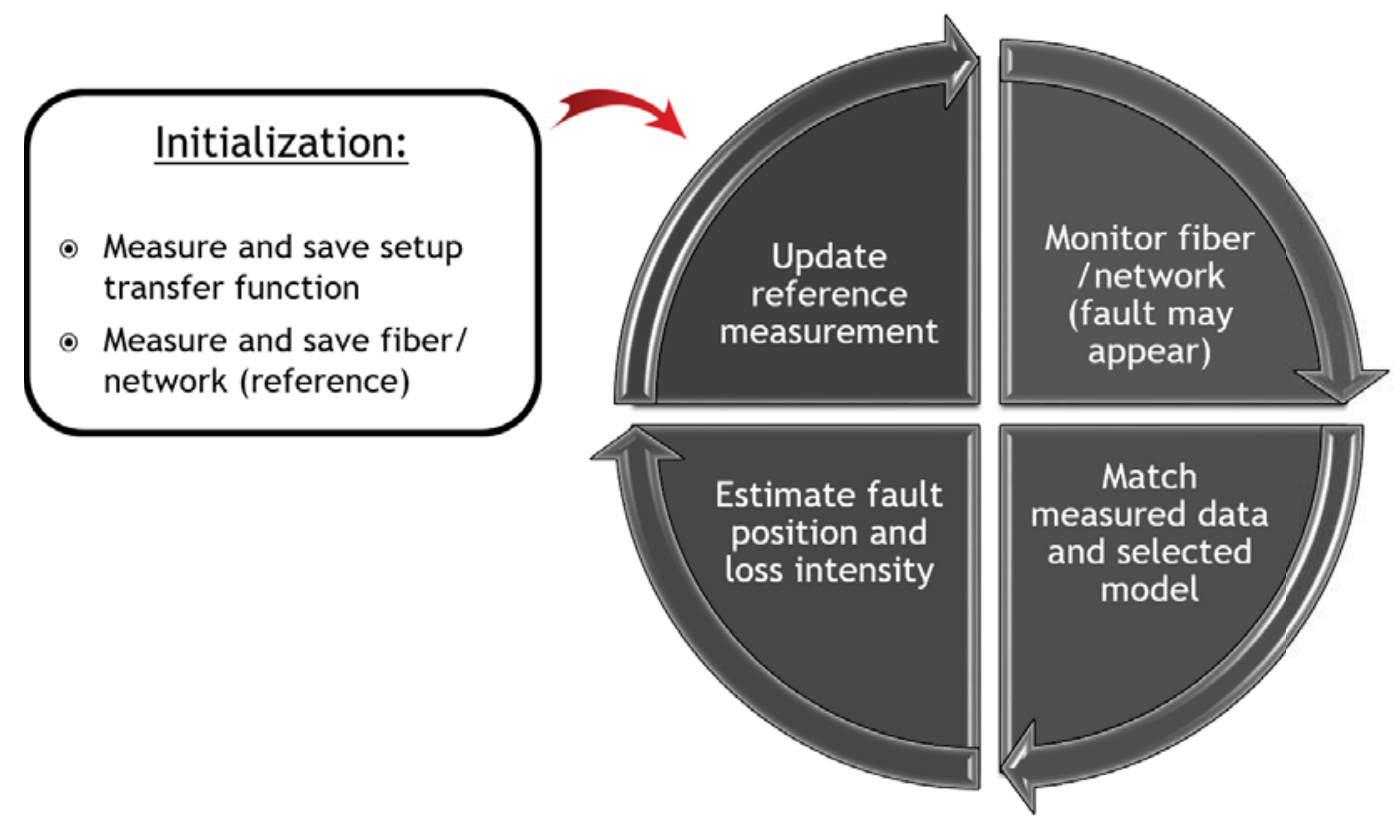

Figure 4.2: Proposed scheme for the monitoring comparison process

The experimental setup required for testing the proposed solution is presented in the next section, considering a generic fiber link to be monitored. Some information about the fiber characteristics are needed for the mathematical model and the estimation process, including a measurement or data from its prior state, since the method designed is used to find the occurrence of a new fault.

The mathematical model for the complex (amplitude and phase) detected signal and corresponding computational specifications are described in the following chapter, along with a brief discussion about possible variations and limitations. The model for the detected signal described considers the cases of a single point-to-point fiber link and of a branched TDM-PON. A WDM-PON can be monitored as a set of single links, one over each wavelength, which doesn't incur additional cost since the data signal is also modulated over the same set of wavelengths.

Employing more complex optimization techniques is likely to yield better and faster results, but further improvements on the optimization solution used are left outside the scope of this research. 
A chapter presenting simulated results with a variety of fault scenarios as well as some simulation statistics is included. Experimental results empirically representing the fiber link were executed in a laboratory setting for both pointto-point and branched emulated links and are presented afterwards. These results are compared to measurements of the same fibers performed with the aid of a commercially available OTDR in order to corroborate the efficiency of the technique.

\section{2}

\section{Setup}

Like many existing solutions, the fiber monitoring technique proposed in this research is based on launching a modulated optical carrier into a fiber (or fiber network) and evaluating the backscattered signal detected at the same end of it through an optical circulator. In particular, the method evaluated is similar to an OFDR solution, in that a CW laser is used and the amplitude of a sub-carrier selected for monitoring is modulated by a frequency swept sinusoidal tone.

Since Rayleigh backscatter doesn't affect the signal properties (including modulation), sweeping the base band tone frequency of the modulating signal and detecting the backscattered signal allows for the transfer function of the setup - system response as a function of the frequency, $S(f)$ - to be acquired. The transfer function of the fiber or network under test alone can be obtained by removing the effect of the combined transfer function of the other elements in the monitoring setup.

Figure 4.3 represents the block diagram of the experimental setup used consisting of a directly modulated laser diode (LD) as the light source, a circulator (C), a photo-detector (PD) including a Pre-Amplifier and a Lock-in Amplifier. The Lock-in Amplifier generates the modulating electronic sine signal with frequency sweep varying from $100 \mathrm{~Hz}$ to $100 \mathrm{kHz}$ and determines the amplitude and relative phase of the backscattered signal detected. This measurement corresponds to the complex frequency response of the system+fiber, namely the combined transfer function.

When a reflective event is induced at port 2 of the circulator, the transfer function measured in the manner described here corresponds to the effect of the setup equipment. This data is used to remove the impact $H(f)$ of the monitoring system elements in order to obtain the desirable transfer function for the fiber or network under test only. This is necessary because the mathematical pattern used for determining fault parameters is based on the properties of Rayleigh backscatter and models the response of the optical 
fiber or network exclusively.

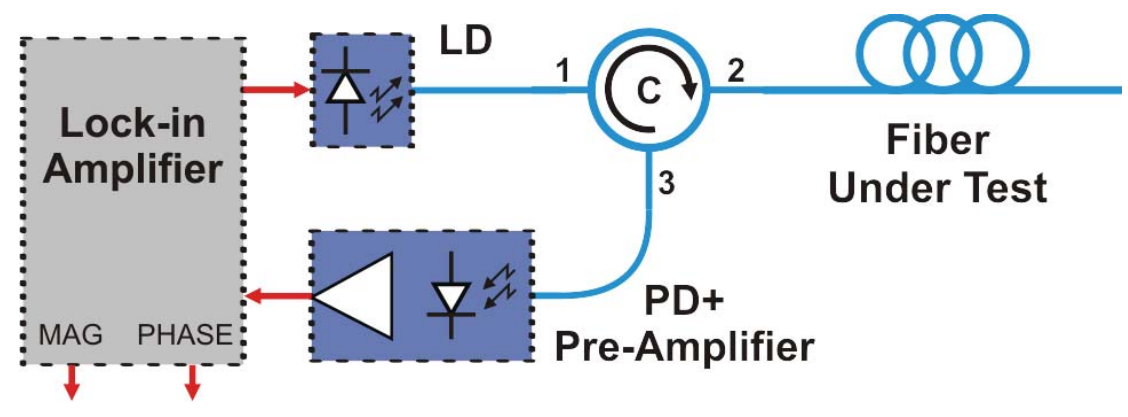

Figure 4.3: Block diagram of the designed experimental setup for base band sub-carrier frequency sweep monitoring 


\section{5}

\section{Mathematical Model}

The data obtained from the Lock-In Amplifier (after properly treated for the effect of the unwanted setup elements) is compared with the appropriate mathematical model for the fiber from which the link parameters can be estimated. This way, a good approximation for the position (distance from the monitoring end) and intensity of a single additional fault (bend, break,...) can be determined, provided that previous faults (connectors, equipment,...) and other parameters such as length of the link, average attenuation, group refraction index are known. The estimation process is done by fitting the data from the measured transfer function to the non-linear mathematical model representing the physical response expected for the fiber link under test.

Once the expected behavior of the backscattered response signal has been modeled by a mathematical equation, a range of curve fitting optimization algorithms may be employed to match the data to the curve which represents the link response, such that the unknown location and intensity parameters of any given new fault can be estimated. It is important to note that the signal model developed in this research approximates the complex valued information measured by the Lock-in Amplifier shown in figure 4.3 as a function of the swept modulation frequency.

In order to achieve the desired results, the approach selected in this study was to apply a non-linear least squares curve fitting algorithm. MATLAB ${ }^{\circledR}$ program was chosen for this purpose, since it provides a range of reliable curve-fitting algorithms and great ease of implementation for simulation and application purposes. The analysis presented here is realized by employing the default version of the non-linear curve-fitting algorithm embedded in the MATLAB $^{\circledR}$ Optimization Toolbox ${ }^{\mathrm{TM}}$ called lsqcurvefit, which consists of a non-linear least squares solver that finds the coefficients $\mathbf{x}$ to solve the minimization problem

$$
\min _{\mathbf{x}}\|F(\mathbf{x}, x d a t a)-y d a t a\|_{2}^{2}=\min _{\mathbf{x}} \sum_{i}\left(F\left(\mathbf{x}, x d a t a_{i}\right)-y d a t a_{i}\right)^{2}
$$

where a known output ydata is observed for a given input xdata. In simpler words, the algorithm matches a function defined by the user $F(\mathbf{x}, x d a t a)$ to 
experimental results ydata obtained for xdata input. Within this study, the function $F(\mathbf{x}, x d a t a)$ corresponds to the mathematical model developed for the system response being analyzed, $x$ data is the swept base band frequency applied by the Lock-in Amplifier and ydata is the complex valued (amplitude and phase) data obtained at the output of the Lock-in Amplifier as described in the experimental setup.

For the purpose of this research, $\mathbf{x}$ is a vector including the fiber properties to be estimated, that is fault location and loss factor intensity. For monitoring of a TDM-PON, initially $\mathbf{x}$ was considered as a sparse $N \times 2$ matrix where the rows represent the branches and the columns represent fault position and intensity, such that a single new fault is described with non-zero values only in the row corresponding to the faulty branch. The optimization with this solution often failed to converge to a minimum value, so another method was required. An optimization in which $N+1$ alternative scenarios for fault location, namely in the feeder fiber or in each of the $N$ branches of the splitter, is considered where the estimation that corresponds to the least residual value in the minimization algorithm is chosen.

The goal function $F(\mathbf{x}, x$ data $)$ of choice to be fit in the case of the fiber network monitoring technique in question here is presented and discussed in detail next. First a point-to-point (single fiber) link is considered and the mathematical model for it is defined as the goal function. Later, the model is extended for branched fiber network (PON) and the results for this more complete solution are discussed.

The optimization method selected has shown good results for both simulated and experimental analysis, but may possibly be improved upon. The choice of best optimization algorithm is left for future work outside the scope of this research, nonetheless some discussion on the performance and limitations of the algorithm employed will be offered along with the simulations results and statistical analysis.

\section{1}

\section{Single Link Model}

Consider a low-frequency tone with angular frequency $\omega,(\omega=2 \pi f)$ is used to directly modulate a laser, within its region of linear operation. The optical signal launched into the fiber will carry the low-frequency tone modulation, as will the backscattered signal resulting from it. For a given detector with detectivity $D$, the backscattered signal detected and sent to the Lock-in Amplifier results in an electrical signal measured over time $v(t)=D A \cos (\omega t)$, where $A$ is proportional to the intensity of the backscattered 


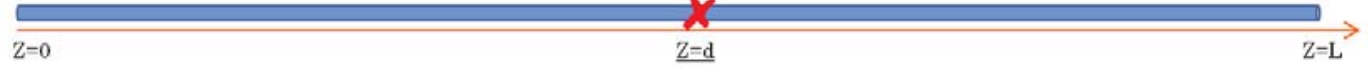

Figure 5.1: Single fiber link of length $L$ and single fault at position $d$

optical field [85]. The gain of the pre-amplifier is considered to be inserted in the detectivity $D=G D^{\prime}$ or to be equal to unity. Either way, its effect will be treated by the setup transfer function $H(f)$ as previously mentioned and will not influence the mathematical model.

The signal intensity arriving back at the Lock-in Amplifier is given by the the sum of punctual reflections (with reflectivity $R_{i}$ ) that may occur along the fiber at corresponding positions $\left(d_{i}\right)$ measured as the distance to the fiber input, as well as the incoherent sum of light scattered from differential positions along the link resulting from Rayleigh backscattering (RBS).

The mathematical model for the backscattered and back-reflected signal is described by equation 5-1, where $P_{0}$ represents the optical power at the input to the fiber under test and $C$ corresponds to the Rayleigh backscatter coefficient assumed to be constant within the length $L$ of the fiber. The coefficient $K=k \omega+j \alpha$ corresponds to the complex wave vector of the modulating signal, including the fiber attenuation coefficient in its imaginary part, and with $k=n / c$, where $n$ is the average refraction index in the fiber.

$$
s(t)=\sum_{i} R_{i} D P_{0} e^{j\left(2 K d_{i}-\omega t\right)}+\int_{0}^{L} D C P_{0} e^{j(2 K z-\omega t)} \mathrm{d} z
$$

From equation 5-1 it is clear that punctual reflections may be treated separately, so herein forth they are neglected in this work, so that the present research is focused in evaluating the backscattered light component which is required for the estimation of the non-reflective faults that may affect quality of service. Also, to simplify the analysis, let's consider the constant amplitude factor $A_{0}=D C P_{0}$. Equation 5-1 is therefore simplified to:

$$
s(t)=\int_{0}^{L} A_{0} e^{j(2 K z-\omega t)} \mathrm{d} z
$$

Equation 5-2 describes the measured signal in a point-to-point link without any existing faults, which corresponds to the reference signal $s_{0}(t)$. Solving the integral yields:

$$
s_{0}(t)=A_{0} \frac{\sin (K L)}{K} e^{j(K L-\omega t)}
$$

Considering then the simple case where a single non-reflective fault occurs at a position $d$ measured from the input to the fiber, with corresponding loss factor $(1-\delta)$, meaning that a fraction $\delta$ of the light intensity continues on from that point (and is scattered along the remainder of the fiber length), the 
detected signal $s(t)$ is described now as:

$$
s(t)=\int_{0}^{d} A_{0} e^{j(2 K z-\omega t)} \mathrm{d} z+\delta^{2} \int_{d}^{L} A_{0} e^{j(2 K z-\omega t)} \mathrm{d} z
$$

It is clear that the first integral describes the light backscattered from the first section of the fiber, up to the point where the fault occurred, while the second integral corresponds to the fraction of light backscattered from the section of the fiber after the fault position, until the end of the fiber as shown in equation 5.1. The fault intensity factor $\delta$ affects the second integral twice as the light travels both ways, hence the squared factor, assuming that the fault effect to be symmetric.

Equation 5-4 can be simplified by considering the property of the additivity of integration shown in equation 5-5, to obtain the equation 5-6 that models a single fault event in a single fiber as a function of time. Solving the integrals yields equation 5-7 which represents the detected signal intensity as a function of time.

$$
\begin{gathered}
\int_{d}^{L} f(z) \mathrm{d} z=\int_{0}^{L} f(z) \mathrm{d} z-\int_{0}^{d} f(z) \mathrm{d} z \\
s(t)=\delta^{2} \int_{0}^{L} A_{0} e^{j(2 K z-\omega t)} \mathrm{d} z+\left(1-\delta^{2}\right) \int_{0}^{d} A_{0} e^{j(2 K z-\omega t)} \mathrm{d} z \\
s(t)=\delta^{2} A_{0} \frac{\sin (K L)}{K} e^{j(K L-\omega t)}+\left(1-\delta^{2}\right) A_{0} \frac{\sin (K d)}{K} e^{j(K d-\omega t)}
\end{gathered}
$$

The result described in equation 5-7 may be interpreted as the sum of two signals: one representing the fraction of light that travels through the whole fiber length, thus also through the fault, which contributes with a factor $\delta^{2}$ to the total backscattered light; the other composed of light that travels only through the first section of the link up to the fault location and contributes to the total backscattered light with a factor $\left(1-\delta^{2}\right)$. Note that the first signal is proportional to the reference signal $s_{0}(t)$ described in equation 5-3.

The signal $s(t)$ has a clear form of a phasor (a phase vector), $s(t)=$ $S(f) e^{-j \omega t}$, and henceforth it will be dealt with by its complex time-invariant factor $S(f)$. In this manner, equation 5-7 can be represented by its the timeinvariant part described by equation 5-8. This equation will be used with an optimization algorithm to fit the experimental data, provided the link has a single non-reflective fault with unknown position and intensity and the fiber length, refraction index and attenuation are known parameters.

The fact that equation 5-8 is composed of two signals, one of them proportional to $S_{0}(f)$ (the time-invariant part of the reference signal) allows for the alternative to the original method proposed, where the measured signal is subtracted from a reference measurement and the minimization process 
considers $\Delta S(f)=S_{0}(f)-S(f)$ as a goal function. This result leads to a simpler optimization problem depending on the reference signal measurement and the group refraction index only as suggested by 5-9 (see Appendix A for more detailed analysis).

$$
\begin{aligned}
S(f)= & \delta^{2} A_{0} \frac{\sin (K L)}{K} e^{j K L}+\left(1-\delta^{2}\right) A_{0} \frac{\sin (K d)}{K} e^{j K d} \\
\Delta S(f) & =S_{0}(f)-S(f) \\
& =\left(1-\delta^{2}\right) S_{0}(f)-\left(1-\delta^{2}\right) A_{0} \frac{\sin (K d)}{K} e^{j K d}
\end{aligned}
$$

Equation 5-8 may be extended to consider an arbitrary number $M$ of $n o n$ reflective faults as described in figure 5.2. This result is important in order to estimate the mathematical model for a link with existing known faults (bends, connectors, ...) whose impact is previously known and dealt with and where it is necessary to monitor the occurrence of a new fault that may disrupt the service level. The expanded version of the model is presented in equation 510 and a more generic form is given by equation $5-11$, where $\delta_{0}=1(0 \mathrm{~dB})$, $d_{M+1}=L$ and $\delta_{M+1}=0$ (end of fiber).

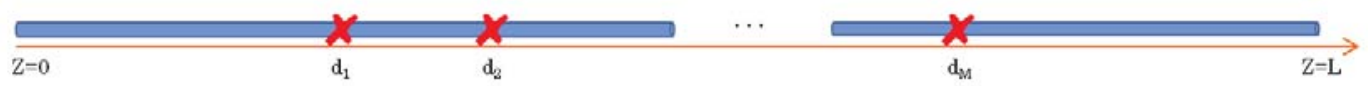

Figure 5.2: Single fiber link with multiple faults

$$
\begin{aligned}
S_{M}(f) & =A_{0}\left\{\sum_{m=1}^{M}\left[\prod_{i=0}^{m-1} \delta_{i}^{2}\right]\left(1-\delta_{m}^{2}\right) \frac{\sin \left(K d_{m}\right)}{K} e^{j K d_{m}}\right. \\
& \left.+\left[\prod_{i=0}^{M} \delta_{i}^{2}\right] \frac{\sin (K L)}{K} e^{j K L}\right\} \\
S_{M}(f) & =A_{0} \sum_{m=1}^{M+1}\left[\prod_{i=0}^{m-1} \delta_{i}^{2}\right]\left(1-\delta_{m}^{2}\right) \frac{\sin \left(K d_{m}\right)}{K} e^{j K d_{m}}
\end{aligned}
$$

Equation 5-11 is implemented as the optimization goal function in a MATLAB ${ }^{\circledR}$ script to perform simulations of various fault scenarios in a pointto-point link: single fault in a clear link, new fault in a link with known existing faults, new fault in a link with small unknown existing faults, among other settings. Simulations are used to evaluate the performance for various positions relative to the origin and different fault loss factor intensities. The same function implementation is also used to fit experimentally measured empirical data obtained from the Lock-in Amplifier with the setup described in 
figure 7.2 in order to estimate location and intensity of induced faults. These faults are measured with the aid of a commercial OTDR which provides a reference value to validate the efficiency of the proposed monitoring method and calculate the estimation error.

As mentioned, optimization can also be performed using $\Delta S(f)=$ $S_{0}(f)-S(f)$, where the reference signal $=S_{0}(f)$ corresponds to the measurement of the fiber with $M$ existing faults, in a similar fashion to that described by equation 5-9. In this case, the estimated fault intensity must be adjusted depending on its estimated location. This more complex solution may be achieved by testing for the different possible positions (before the first existing fault, between the first and second existing faults, between the second and third existing faults, and so on) and selecting the best fit based on the lowest value of the residual for the minimization problem. This solution is also discussed further in Appendix A.

\section{2}

\section{PON Model}

In this section, a TDM-PON branched optical network is considered. The monitoring solution for a WDM-PON setup is the same as that for the point-to-point link, where the Wavelength multiplexer presents as an existing known fault proportional to its insertion loss. Monitoring in the latter is done separately for each wavelength along with the corresponding sub-carrier modulated data signal.

For the same monitoring signal and setup described for a point-to-point link to be employed for a PON, the mathematical formulation needs to be extended for describing a branched network. The first step is to describe the backscattered signal detected by the lock-in amplifier $s(t)$ for a network consisting of a feeder line of length $L_{0}$ where an optical power splitter (PS) is located followed by a number $N$ of branches of various lengths $L_{i}$. It is important to note that, for a TDM-PON, the system detects the sum of the light scattered from each branch.

Consider first a simple case where $1: 2$ splitter is used with insertion loss $\delta_{s}$ as described in figure 5.3 without the occurence of any other faults. Equation 5-2 for the detected signal response at the Lock-in Amplifier reflecting this scenario is expressed as:

$$
\begin{aligned}
s(t)= & \int_{0}^{L_{0}} A_{0} e^{j(2 K z-\omega t)} \mathrm{d} z \\
& +\frac{\delta_{s}^{2}}{4} \int_{L_{0}}^{L_{1}} A_{0} e^{j(2 K z-\omega t)} \mathrm{d} z+\frac{\delta_{s}^{2}}{4} \int_{L_{0}}^{L_{2}} A_{0} e^{j(2 K z-\omega t)} \mathrm{d} z
\end{aligned}
$$




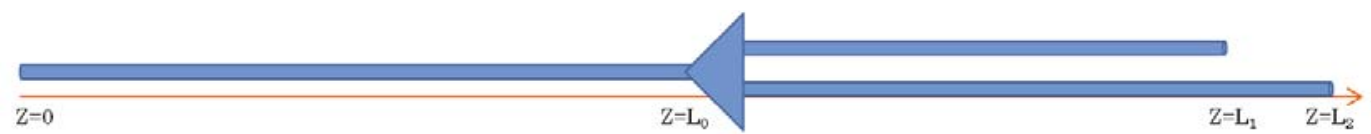

Figure 5.3: PON with $N=2$ and splitter insertion loss $\delta_{s}$

Re-writing equation 5-12, the contribution of the light backscattered from each branch to the detected signal may be analyzed separately as shown in equation 5-13. The contribution from each fiber is proportional to the case of a point-to-point link of total length $L_{i}$ with a single fault at the end of the feeder line (position $L_{0}<L_{i}$ ) with loss intensity equal to the insertion loss of the power splitter.

$$
\begin{aligned}
s(t)= & {\left[\frac{1}{2} \int_{0}^{L_{0}} A_{0} e^{j(2 K z-\omega t)} \mathrm{d} z+\frac{\delta_{s}^{2}}{4} \int_{L_{0}}^{L_{1}} A_{0} e^{j(2 K z-\omega t)} \mathrm{d} z\right] } \\
& +\left[\frac{1}{2} \int_{0}^{L_{0}} A_{0} e^{j(2 K z-\omega t)} \mathrm{d} z+\frac{\delta_{s}^{2}}{4} \int_{L_{0}}^{L_{2}} A_{0} e^{j(2 K z-\omega t)} \mathrm{d} z\right] \\
= & \frac{1}{2}\left[\int_{0}^{L_{0}} A_{0} e^{j(2 K z-\omega t)} \mathrm{d} z+\frac{\delta_{s}^{2}}{2} \int_{L_{0}}^{L_{1}} A_{0} e^{j(2 K z-\omega t)} \mathrm{d} z\right] \\
& +\frac{1}{2}\left[\int_{0}^{L_{0}} A_{0} e^{j(2 K z-\omega t)} \mathrm{d} z+\frac{\delta_{s}^{2}}{2} \int_{L_{0}}^{L_{2}} A_{0} e^{j(2 K z-\omega t)} \mathrm{d} z\right] \\
= & s_{1}(t)+s_{2}(t)
\end{aligned}
$$

Evaluating the light backscattered from each branch separately yields the following results:

$$
\begin{aligned}
& s_{1}(t)=\frac{A_{0}}{2}\left[\left(1-\frac{\delta_{s}^{2}}{2}\right) \frac{\sin \left(K L_{0}\right)}{K} e^{j\left(K L_{0}-\omega t\right)}+\frac{\delta_{s}^{2}}{2} \frac{\sin \left(K L_{1}\right)}{K} e^{j\left(K L_{1}-\omega t\right)}\right] \\
& s_{2}(t)=\frac{A_{0}}{2}\left[\left(1-\frac{\delta_{s}^{2}}{2}\right) \frac{\sin \left(K L_{0}\right)}{K} e^{j\left(K L_{0}-\omega t\right)}+\frac{\delta_{s}^{2}}{2} \frac{\sin \left(K L_{2}\right)}{K} e^{j\left(K L_{2}-\omega t\right)}\right]
\end{aligned}
$$

The total response measured for this scenario is given by the sum of the terms $s_{1}$ and $s_{2}$ in equation 5-14, in agreement with the fact that the reflectometry trace for a PON presents an overlap between the traces of the individual branches. Again, each of the terms presents in the form of a phasor and will be represented by its time-invariant factor $S_{i}(f)$. Finally, the model for a $1: 2$ PON with no additional faults (other that the PS) is given by: 


$$
\begin{aligned}
S(f)= & S_{1}(f)+S_{2}(f) \\
= & \left(1-\frac{\delta_{s}^{2}}{2}\right) A_{0} \frac{\sin \left(K L_{0}\right)}{K} e^{j K L_{0}} \\
& +\frac{\delta_{s}^{2}}{4} A_{0} \frac{\sin \left(K L_{1}\right)}{K} e^{j K L_{1}}+\frac{\delta_{s}^{2}}{4} A_{0} \frac{\sin \left(K L_{2}\right)}{K} e^{j K L_{2}}
\end{aligned}
$$

The model described in equation 5-15 may be easily extended to a network with a large number $N$ of branches such as the one described in figure 3.1. For the case where no faults occur, the contribution from each fiber is given as:

$$
S_{n}(f)=\frac{1}{N}\left[\left(1-\frac{\delta_{s}^{2}}{N}\right) A_{0} \frac{\sin \left(K L_{0}\right)}{K} e^{j K L_{0}}+\frac{\delta_{s}^{2}}{N} A_{0} \frac{\sin \left(K L_{n}\right)}{K} e^{j K L_{n}}\right]
$$

The total signal detected at the input to the fiber is modeled by the sum of $N$ contributions for each branched fiber, which represents the reference signal for this PON. The occurrence of one or more fault in any of the branches is derived next and may be easier to analyze by subtracting the expected result obtained in equation 5-17.

$$
\begin{aligned}
S(f) & =\sum_{i=1}^{N} S_{i}(f) \\
& \left.=\left(1-\frac{\delta_{s}^{2}}{N}\right) A_{0} \frac{\sin \left(K L_{0}\right)}{K} e^{j K L_{0}}+\frac{\delta_{s}^{2}}{N^{2}} \sum_{i=1}^{N} A_{0} \frac{\sin \left(K L_{i}\right)}{K} e^{j K L_{i}}\right]
\end{aligned}
$$

This analysis may be extended to consider the presence of faults in the branches (either existing or new). Considering first the simplest case where a single fault with corresponding loss factor $\delta$ occurs in one of the branches (branch $n$ ) at a position $d$ from the input to the feeder fiber, the contribution from the light backscattered through the corresponding branch to the total detected signal is described by:

$$
\begin{aligned}
S_{n}(f)= & \frac{1}{N}\left[\left(1-\frac{\delta_{s}^{2}}{N}\right) A_{0} \frac{\sin \left(K L_{0}\right)}{K} e^{j K L_{0}}\right. \\
& \left.+\frac{\delta_{s}^{2}\left(1-\delta^{2}\right)}{N} A_{0} \frac{\sin (K d)}{K} e^{j K d}+\frac{\delta_{s}^{2} \delta^{2}}{N} A_{0} \frac{\sin \left(K L_{n}\right)}{K} e^{j K L_{n}}\right]
\end{aligned}
$$

Using the result of equation 5-18 to substitute the contribution of the $n^{\text {th }}$ network branch in equation 5-17 yields the result presented in equation 5-19. It is clear that this signal corresponds to the combination of a no-fault 
scenario with two additional terms balanced by the fault loss factor $\delta$ : one term dependent on parameter $d$ corresponding to the location of the fault and the other term related to the length of the corresponding branch $L_{n}$.

$$
\begin{aligned}
S(f)= & \sum_{i=1}^{N} S_{i}(f) \\
= & \left(1-\frac{\delta_{s}^{2}}{N}\right) A_{0} \frac{\sin \left(K L_{0}\right)}{K} e^{j K L_{0}}+\frac{\delta_{s}^{2}}{N^{2}} \sum_{i=1}^{N} A_{0} \frac{\sin \left(K L_{i}\right)}{K} e^{j K L_{i}} \\
& +\frac{\delta_{s}^{2}\left(1-\delta^{2}\right)}{N^{2}} A_{0} \frac{\sin (K d)}{K} e^{j K d}-\frac{\delta_{s}^{2}\left(1-\delta^{2}\right)}{N^{2}} A_{0} \frac{\sin \left(K L_{n}\right)}{K} e^{j K L_{n}}
\end{aligned}
$$

For a slightly more complicated case where more than one faults (including known elements such as connectors, splices,...) occur throughout the fiber link corresponding to the $n^{\text {th }}$ branch of the network, the result from equation 5-11 is applied to the contribution from this branch which becomes:

$$
\begin{aligned}
S_{n}(f)= & \frac{1}{N}\left\{\left(1-\frac{\delta_{s}^{2}}{N}\right) A_{0} \frac{\sin \left(K L_{0}\right)}{K} e^{j K L_{0}}\right. \\
& +\frac{\delta_{s}^{2}}{N} \sum_{m=1}^{M}\left[\prod_{i=0}^{m-1} \delta_{i}^{2}\right]\left(1-\delta_{m}^{2}\right) A_{0} \frac{\sin \left(K d_{m}\right)}{K} e^{j K d_{m}} \\
& \left.+\frac{\delta_{s}^{2}}{N}\left[\prod_{i=1}^{M} \delta_{i}^{2}\right] A_{0} \frac{\sin \left(K L_{n}\right)}{K} e^{j K L_{n}}\right\}
\end{aligned}
$$

The total detected signal is composed, once again, of a solution for the no-fault scenario with $M$ additional terms corresponding to the location of the known faulty elements $\left\{d_{1}, d_{2}, \ldots, d_{M}\right\}$, where $M$ is the number of faults in the $n^{\text {th }}$ branch, and the length $L_{n}$ of the branch where these known faults are located.

$$
\begin{aligned}
S(f)= & \left(1-\frac{\delta_{s}^{2}}{N}\right) A_{0} \frac{\sin \left(K L_{0}\right)}{K} e^{j K L_{0}}+\frac{\delta_{s}^{2}}{N^{2}} \sum_{i=1}^{N} A_{0} \frac{\sin \left(K L_{i}\right)}{K} e^{j K L_{i}} \\
& +\frac{\delta_{s}^{2}}{N^{2}} \sum_{m=1}^{M}\left[\prod_{i=0}^{m-1} \delta_{i}^{2}\right]\left(1-\delta_{m}^{2}\right) A_{0} \frac{\sin \left(K d_{m}\right)}{K} e^{j K d_{m}} \\
& -\frac{\delta_{s}^{2}}{N^{2}}\left(1-\left[\prod_{i=1}^{M} \delta_{i}^{2}\right]\right) A_{0} \frac{\sin \left(K L_{n}\right)}{K} e^{j K L_{n}}
\end{aligned}
$$

Considering now a new case where separate faults with corresponding loss intensity $\delta_{p}$ and $\delta_{q}$ occur in different branches $m$ and $n$ and at positions $d_{p}$ and $d_{q}$ from the input to the feeder fiber, respectively, as shown in figure 5.4. The contribution from each faulty branch may be described as in equation 
5-18 and the total response is written as:

$$
\begin{aligned}
& S(f)=\left(1-\frac{\delta_{s}^{2}}{N}\right) A_{0} \frac{\sin \left(K L_{0}\right)}{K} e^{j K L_{0}}+\frac{\delta_{s}^{2}}{N^{2}} \sum_{i=1}^{N} A_{0} \frac{\sin \left(K L_{i}\right)}{K} e^{j K L_{i}} \\
& +\frac{\delta_{s}^{2}\left(1-\delta_{p}^{2}\right)}{N^{2}} A_{0}\left[\frac{\sin \left(K d_{p}\right)}{K} e^{j K d_{p}}-\frac{\sin \left(K L_{m}\right)}{K} e^{j K L_{m}}\right] \\
& +\frac{\delta_{s}^{2}\left(1-\delta_{q}^{2}\right)}{N^{2}} A_{0}\left[\frac{\sin \left(K d_{q}\right)}{K} e^{j K d_{q}}-\frac{\sin \left(K L_{n}\right)}{K} e^{j K L_{n}}\right]
\end{aligned}
$$

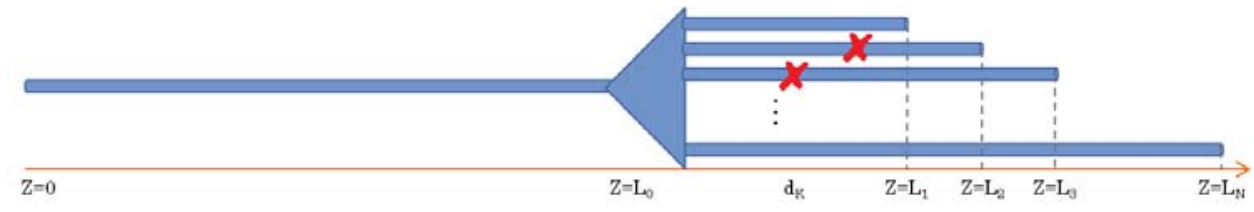

Figure 5.4: PON with 2 faults in separate branches

The result described in equation 5-22 may be extended for any number $N^{\prime} \leq N$ of faulty branches with a single loss each, with loss intensity factor $\delta_{n^{\prime}}$ at a distance $d_{n^{\prime}}$ from the input to the feeder fiber, yielding the detected signal expressed by equation 5-23 at the output of the Lock-in Amplifier.

$$
\begin{aligned}
S(f)= & \left(1-\frac{\delta_{s}^{2}}{N}\right) A_{0} \frac{\sin \left(K L_{0}\right)}{K} e^{j K L_{0}}+\frac{\delta_{s}^{2}}{N^{2}} \sum_{i=1}^{N} A_{0} \frac{\sin \left(K L_{i}\right)}{K} e^{j K L_{i}} \\
& +\frac{\delta_{s}^{2}}{N^{2}} \sum_{n^{\prime}=1}^{N^{\prime}}\left(1-\delta_{n^{\prime}}^{2}\right) A_{0}\left[\frac{\sin \left(K d_{n^{\prime}}\right)}{K} e^{j K d_{n^{\prime}}}-\frac{\sin \left(K L_{n^{\prime}}\right)}{K} e^{j K L_{n^{\prime}}}\right]
\end{aligned}
$$

The combined results from 5-21 and 5-23 leads to the universal case where multiple faults may simultaneously occur in the same link as well as in different branches of the network modeled in equation 5-24. This result represents the desired mathematical model of a realistic scenario, where there may be known connectors, bends and other faults in any or all branches and the goal of the monitoring solution is to detect and measure a new fault within this known setting.

$$
\begin{aligned}
S(f)= & \left(1-\frac{\delta_{s}^{2}}{N}\right) A_{0} \frac{\sin \left(K L_{0}\right)}{K} e^{j K L_{0}}+\frac{\delta_{s}^{2}}{N^{2}} \sum_{i=1}^{N} A_{0} \frac{\sin \left(K L_{i}\right)}{K} e^{j K L_{i}} \\
& +\frac{\delta_{s}^{2}}{N^{2}} \sum_{n=1}^{N^{\prime}} \sum_{m=1}^{M}\left(\prod_{i=0}^{m-1} \delta_{n i}^{2}\right)\left(1-\delta_{n m}^{2}\right) A_{0} \frac{\sin \left(K d_{n m}\right)}{K} e^{j K d_{n m}} \\
& +\frac{\delta_{s}^{2}}{N^{2}} \sum_{n=1}^{N^{\prime}}\left(1-\prod_{i=1}^{M} \delta_{n i}^{2}\right) A_{0} \frac{\sin \left(K L_{n}\right)}{K} e^{j K L_{n}}
\end{aligned}
$$


Considering now the occurrence of a new fault at a position $d$ with intensity $\delta$, two possible scenarios must be considered: either the fault happens in the feeder line, or in one of the branches. If the fault occurs in the feeder line, the model is described by equation $5-25$, where $S_{0}(f)$ represents the reference signal including all previously known faults described like in equation 5-24.

$$
\begin{aligned}
S(f)= & \left(1-\delta^{2}\right) A_{0} \frac{\sin (K d)}{K} e^{j(K d)}+\delta^{2}\left(1-\frac{\delta_{s}^{2}}{N}\right) A_{0} \frac{\sin \left(K L_{0}\right)}{K} e^{j\left(K L_{0}\right)} \\
& +\frac{\delta^{2} \delta_{s}^{2}}{N^{2}} \sum_{n=1}^{N} A_{0} \frac{\sin \left(K L_{n}\right)}{K} e^{j\left(K L_{n}\right)} \\
= & \left(1-\delta^{2}\right) A_{0} \frac{\sin (K d)}{K} e^{j(K d)}+\delta^{2} S_{0}(f)
\end{aligned}
$$

Conversely, if the fault occurs in the $i^{\text {th }}$ branch of the PON, and considering no other faults exist in this branch, its response is modeled by equation 5-26. In the more general case where known faulty elements are present in the branch where the new fault occurs, the model is slightly more complex, in a similar fashion to 5-11 and the closed solution will depend on the position of the new fault relative to the existing ones. To overcome this complexity, each possible location section could be tested separately and the optimization result should be that which results in the least residual of the minimization problem.

$$
\begin{aligned}
S(f)= & \left(1-\frac{\delta_{s}^{2}}{N}\right) A_{0} \frac{\sin \left(K L_{0}\right)}{K} e^{j\left(K L_{0}\right)}+\frac{\delta_{s}^{2}}{N^{2}} \sum_{\substack{n=1 \\
n \neq i}}^{N} A_{0} \frac{\sin \left(K L_{n}\right)}{K} e^{j\left(K L_{n}\right)} \\
& +\frac{\delta_{s}^{2}}{N^{2}}\left(1-\delta^{2}\right) A_{0} e \frac{\sin (K d)}{K} e^{j(K d)}+\frac{\delta^{2} \delta_{s}^{2}}{N^{2}} A_{0} \frac{\sin \left(K L_{i}\right)}{K} e^{j\left(K L_{i}\right)} \\
= & S_{0}(f)+\left(1-\delta^{2}\right) \frac{\delta_{s}^{2}}{N^{2}} A_{0} \frac{\sin (K d)}{K} e^{j(K d)}-\left(1-\delta^{2}\right) \frac{\delta_{s}^{2}}{N^{2}} A_{0} \frac{\sin \left(K L_{i}\right)}{K} e^{j\left(K L_{i}\right)}
\end{aligned}
$$

In either case, the model suggests that knowing the measurement for a reference setting $S_{0}(f)$ results in a much simpler optimization function. Simulations were executed using both methods: the complete mathematical description with vector/matrix solution for the position $d$ and intensity $\delta$ of the new fault, as well as for the differential setting, where $S_{0}(f)$ is known and the measured signal is subtracted from it for optimization. The former doesn't always converge to a solution while the latter was found to be more precise and reliable.

It is important to observe a couple of assumptions that were made in 
modeling the TDM-PON described in this section. Firstly, the model assumes no two branches have the exact same length. It is important that the system be able to differentiate between the branches and simulations indicate that it is sufficient that the difference between any two branches be no less than about $20 \mathrm{~m}$. This is a reasonable assumption as in most branched networks endusers are not likely to be located at the same distance from the central office. Furthermore, some extra fiber may be added to a branch to remove ambiguity of this sort. Secondly, the lengths of each branch as well as the length of the feeder fiber are also presumably known. This value is used in the optimization to determine if the fault occurred in the feeder line or in one of the branches and define the branch that was affected. 


\section{Simulations}

The mathematical model presented in the previous chapter was used to simulate the detected signal resulting from RBS of the sinusoidal signal generated by the Lock-in Amplifier. Simulation of various combinations of link type (point-to-point or PON), length and fault position and intensity were generated with added white complex Gaussian noise to emulate the response of a physical setting. The choice of noise level relative to the signal power was made such that the resulting simulated curves had similar SNR proprieties to those observed in experimental settings.

\section{1}

\section{Simulations using the Complete Mathematical Model}

Initially, the complete form of the mathematical model for the detected signal time invariant part $S(f)$ was used in with a curve fitting algorithm in MATLAB $^{\circledR}$. Some of the results achieved are presented next.

The first link simulated represented a long single fiber link, where fiber length $L=12 \mathrm{~km}$, attenuation factor $\alpha=0.2 \mathrm{~dB} / \mathrm{km}$ and group refraction index $n_{g} \cong 1.47$ are known and a fault occurs at a distance $d=4100 \mathrm{~m}$ from the input. Multiple settings were tested varying the intensity of the loss intensity $\delta$ related to the fault. Additive noise was considered at $25 \mathrm{~dB}$ below the maximal detected power $\left(P_{0}\right)$, resulting in noisy curves with similar SNR to the experimental measurements.

Figure 6.1 shows the behavior of the amplitude of the modeling function for different fault intensities as well as the estimation of the expected curve for detected signal using the proposed method when the data considered is the simulated result with additive white noise. The position and intensity of the faults were estimated by the curve fitting algorithm discussed. The simulation results show very good accuracy (less than $100 \mathrm{~cm}$ and $10^{-2} \mathrm{~dB}$ error) for a single fault detection in an otherwise clear link (no other existing faults). The measure of estimation error presented is given by $\epsilon=\sqrt{(x-\bar{x})^{2}}$.

The optimization algorithm performs curve fitting of the complex simulated data (magnitude and phase) as a function of the frequency to the frequency varying mathematical model developed including a complex exponent 


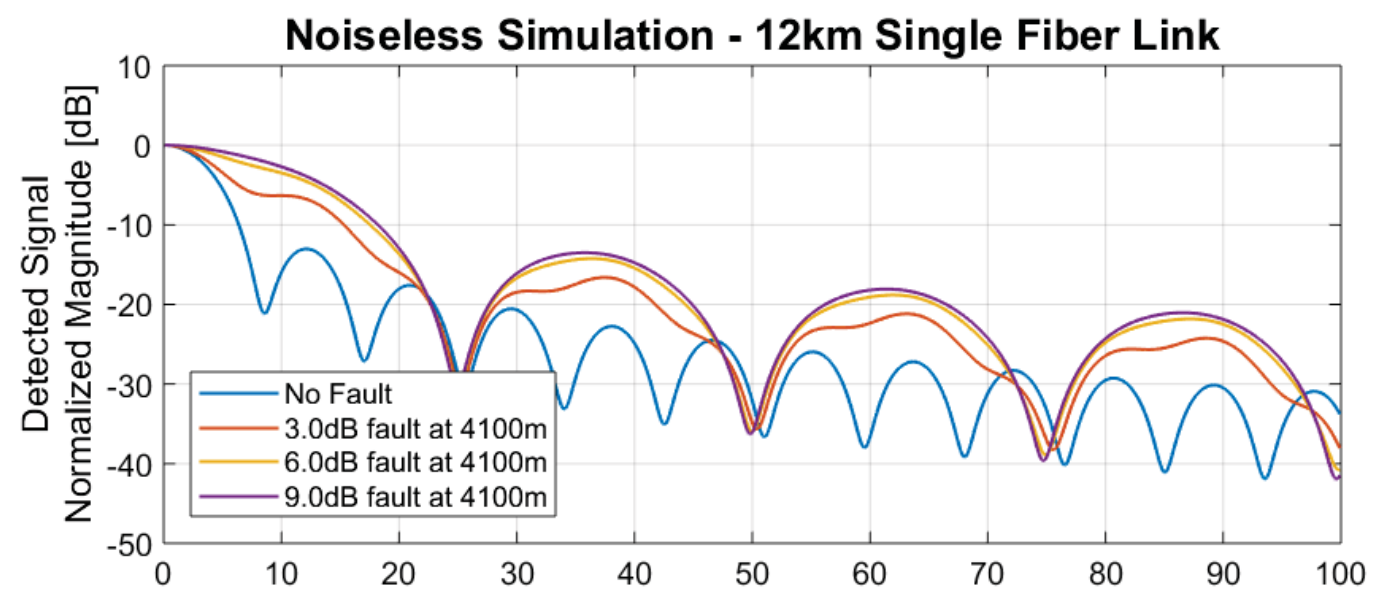

Estimated Values $x$ Noisy Simulation $(S N R=25 d B)$

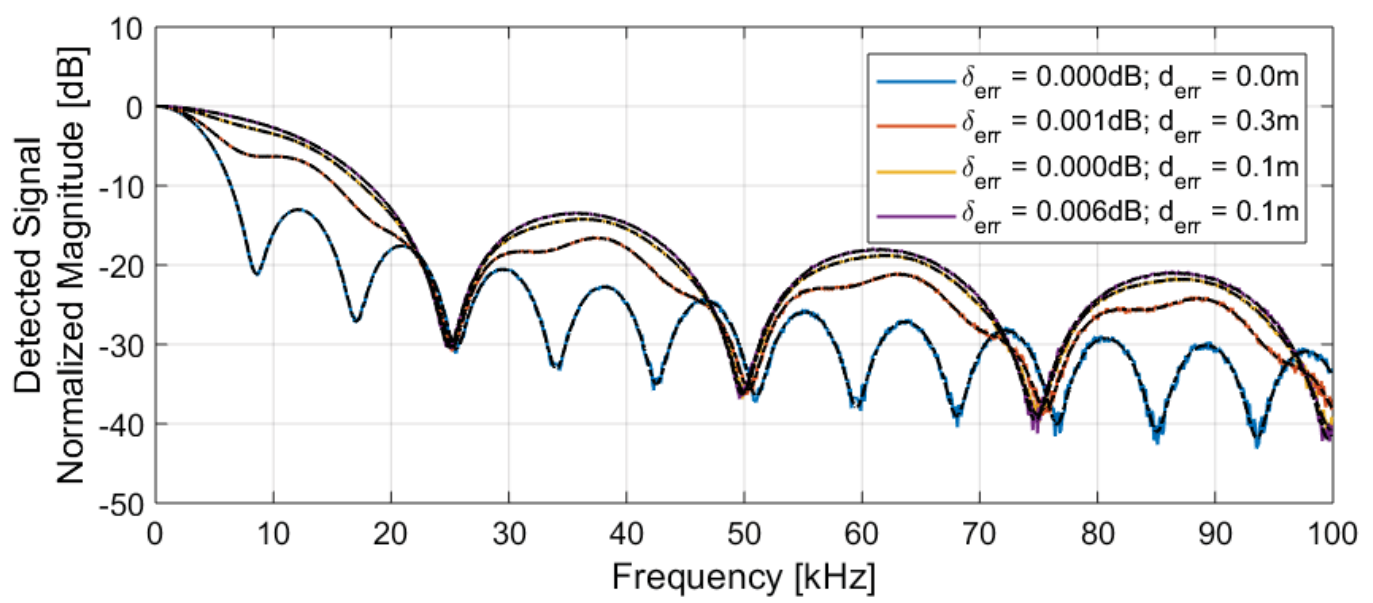

Figure 6.1: Simulation for long single fiber link with varying fault intensity $(d=4100 m)$

term. Both magnitude and phase are fitted simultaneously and the combined result can be seen analyzing a polar plot, where the varying frequency would be represented by the $z$-axis perpendicular to the page. The results of the previous simulation are shown in this alternate form in figure 6.2.

It is important to note that the number of peaks and notches in the response is related to the length of the fiber. In this setting, the link has $12 \mathrm{~km}$ and the signal presents 12 peaks and 11 notches when no loss is considered. For the case where a high loss fault or a break is present (such as the $9 d B$ fault simulated, many peaks vanish and the output has 4 peaks and 4 notches, since the fault occurs after $4 \mathrm{~km}$, approximating the response of a $4 \mathrm{~km}$ long fiber.

The same $12 \mathrm{~km}$ link simulation was repeated considering this time that the position of the fault occurrence was at $d=8300 \mathrm{~m}$. Results are similar to those observed in the previous setting in the order of the estimation errors, indicating that the relative position to the fiber length is not critical for good 

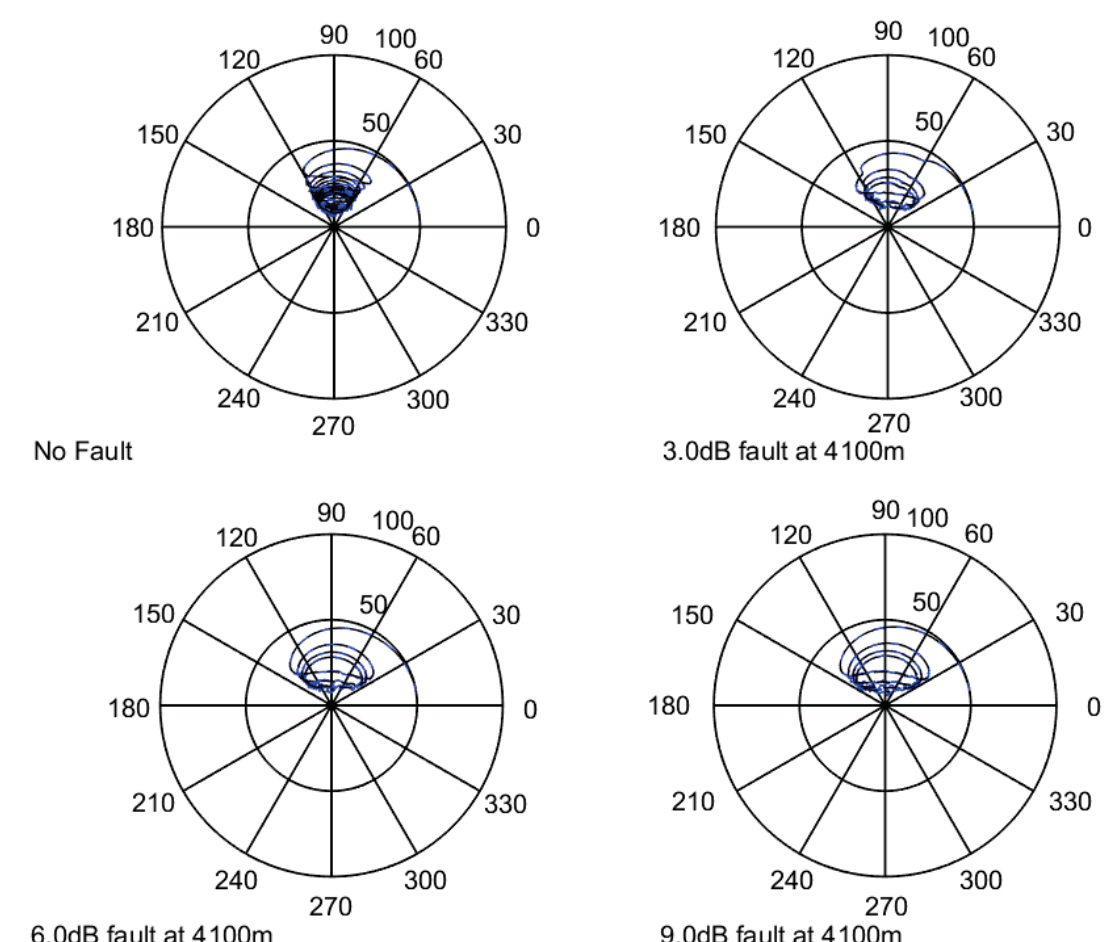

Figure 6.2: Polar plots for long single fiber link simulation $(d=4100 m)$

estimation of the fault parameters. The plots for this simulation shown in figures 6.3 and 6.4 correspond to the comparison of obtained simulated and estimated values for the magnitude and polar views respectively.

Once again, when a large fault is considered like the example of the $9 d B$ fault simulated, the number of peaks and notches is reduced from 12 in the original $12 \mathrm{~km}$ fiber to 8 , since the broken/faulty fiber is equivalent to an $8 \mathrm{~km}$ long link.

Afterwards, a similar simulation was attained for a shorter single fiber link. This kind of link will be the focus of this research hereon in because it can be easily related to local access optical links. Furthermore, the shorter links represent worst case scenarios since less energy is available to be evaluated. In the case simulated, a fiber of length $L=1.1 \mathrm{~km}$ was considered and faults of varying loss factor intensity where simulated at $d=150 \mathrm{~m}$. Other fiber parameters remained the same. In this case a set of known existing faults was included in the model representing existing splice and bend losses.

Noticeably, where the fault considered is large enough that it might be representative of a fiber break, no peaks or notches are visible in the plot range, as is the case of the simulation for $9 d B$ loss at $150 \mathrm{~m}$. The outcome for the signal magnitude in this scenario is presented in figure 6.5, where it is clear that the estimation method yields very good results for short fibers as well as for longer point to point links. The corresponding polar plots including 

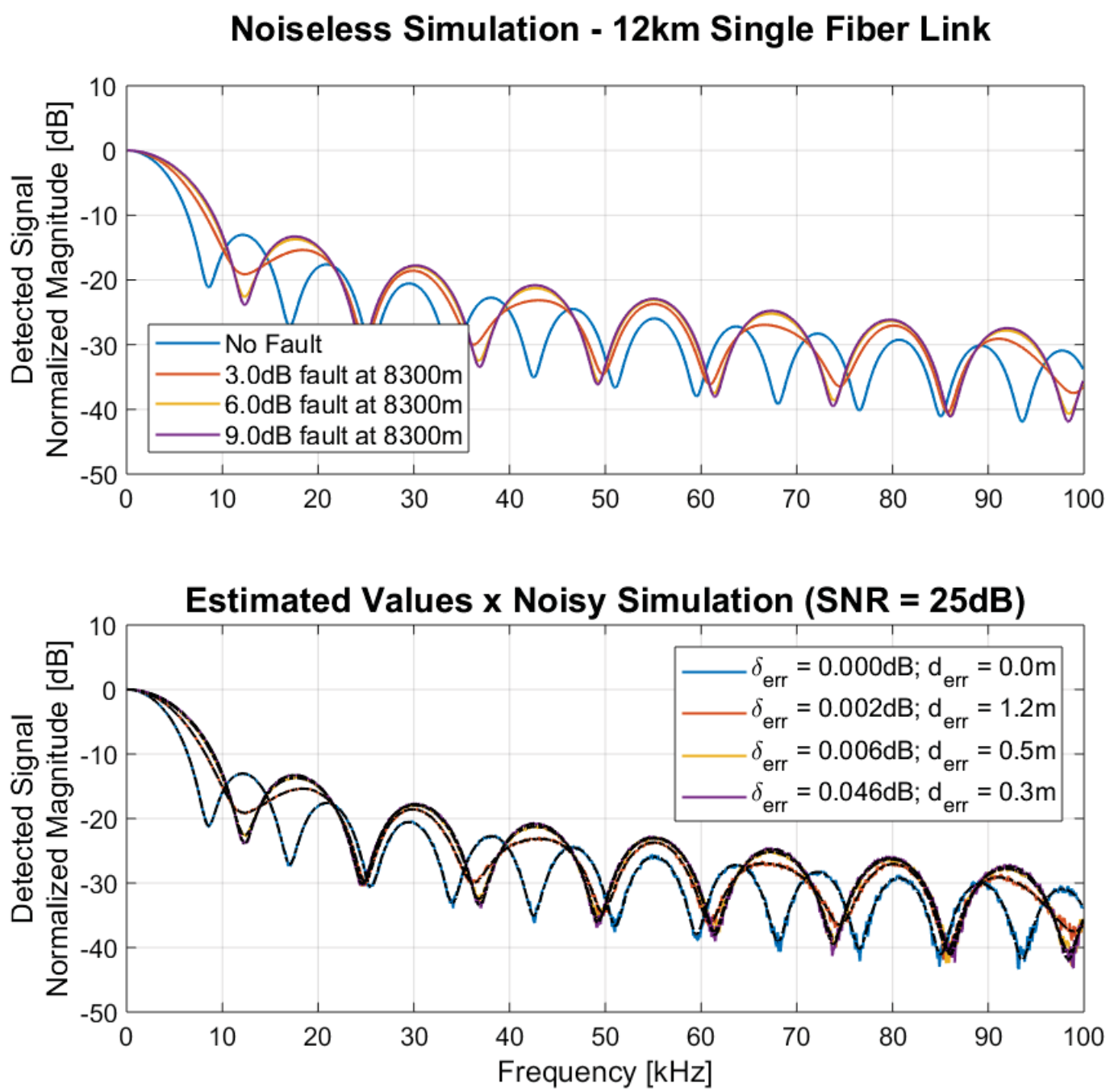

Figure 6.3: Simulation for long single fiber link with varying fault intensity $(d=8300 m)$

magnitude and phase simultaneously are shown in figure 6.6. The output for these simulations speak for the method's capability of dealing with different fault intensities. 

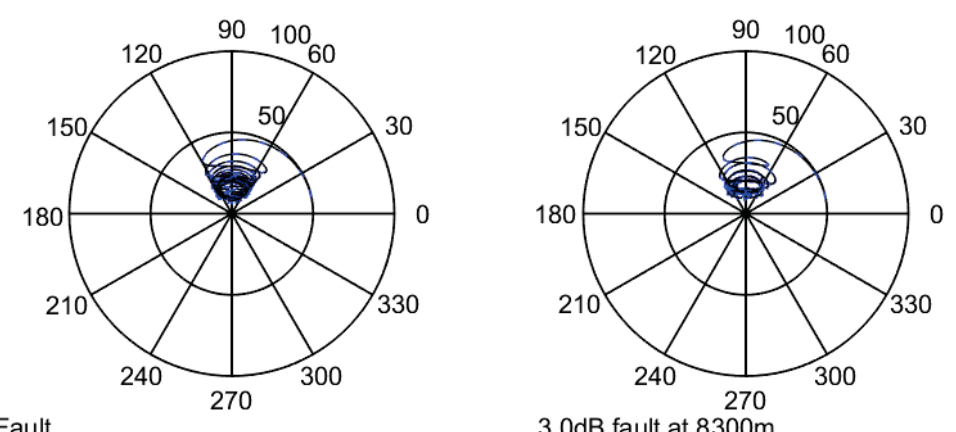

No Fault

$3.0 \mathrm{~dB}$ fault at $8300 \mathrm{~m}$
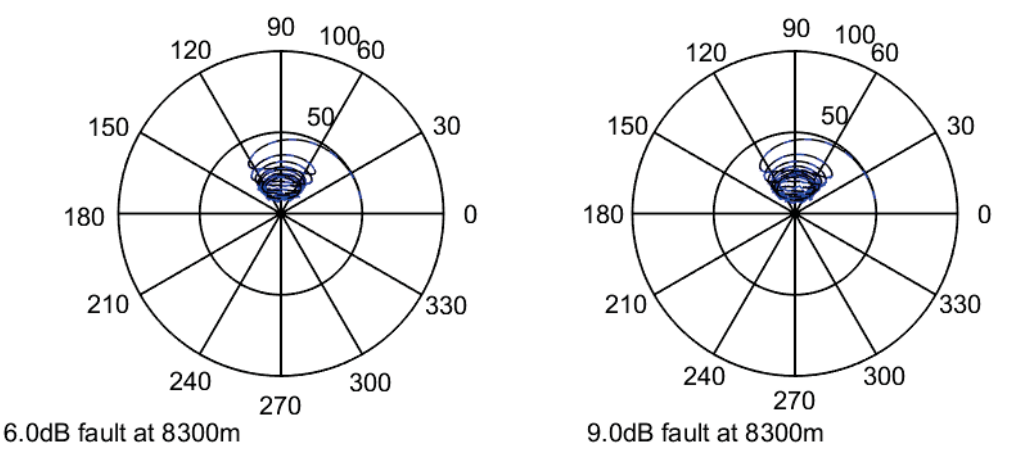

Figure 6.4: Polar plots for long single fiber link simulation $(d=8300 m)$

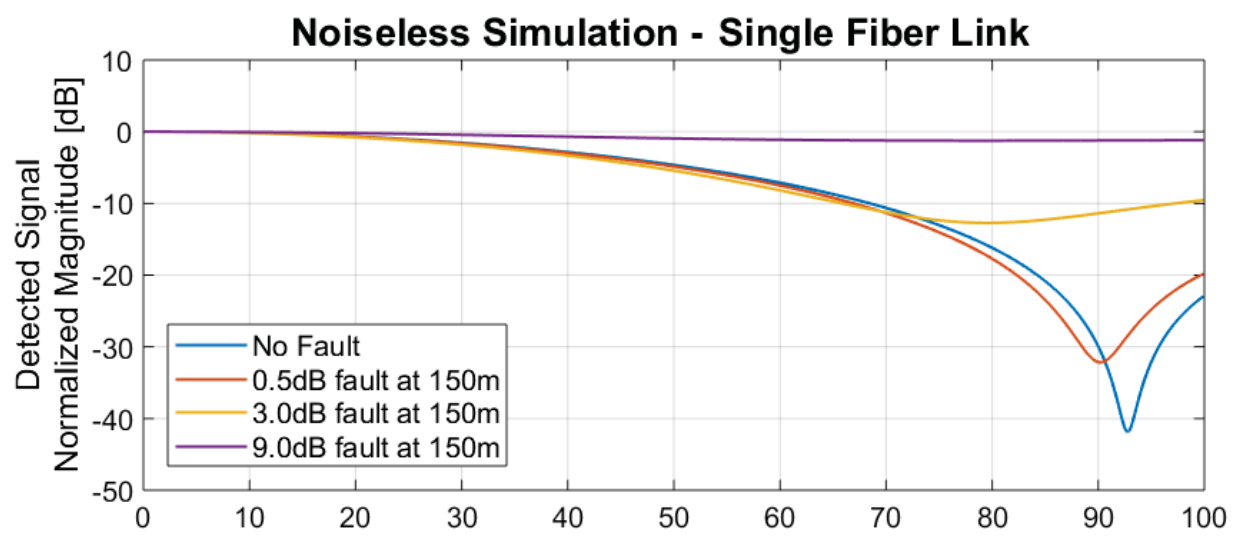

Estimated Values $x$ Noisy Simulation $(S N R=25 d B)$

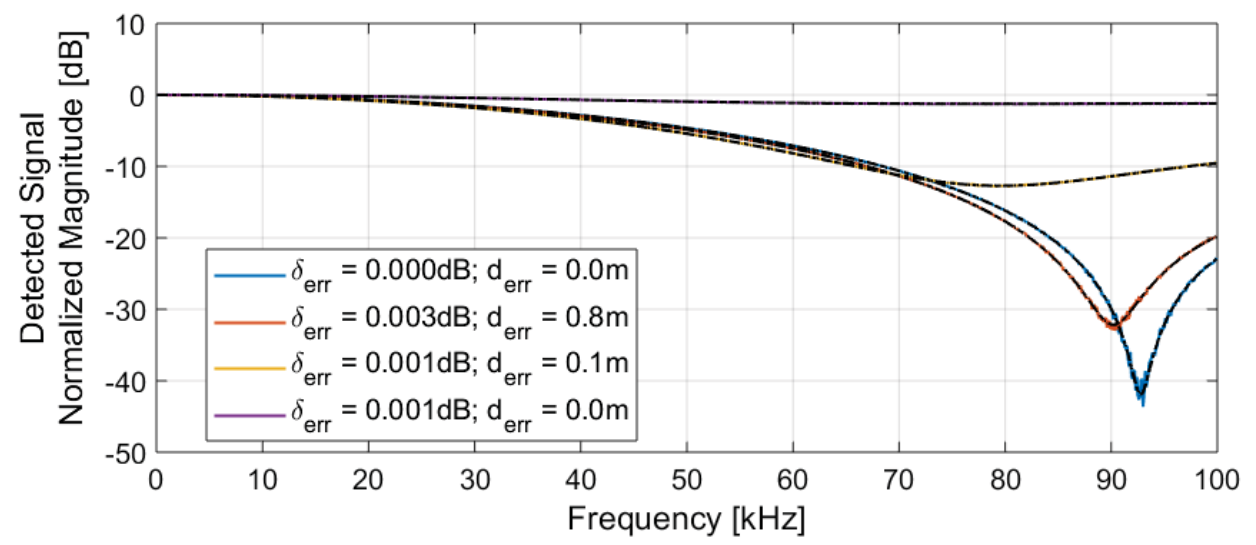

Figure 6.5: Simulation for short single fiber link with varying fault intensity $(d=150 m)$ 

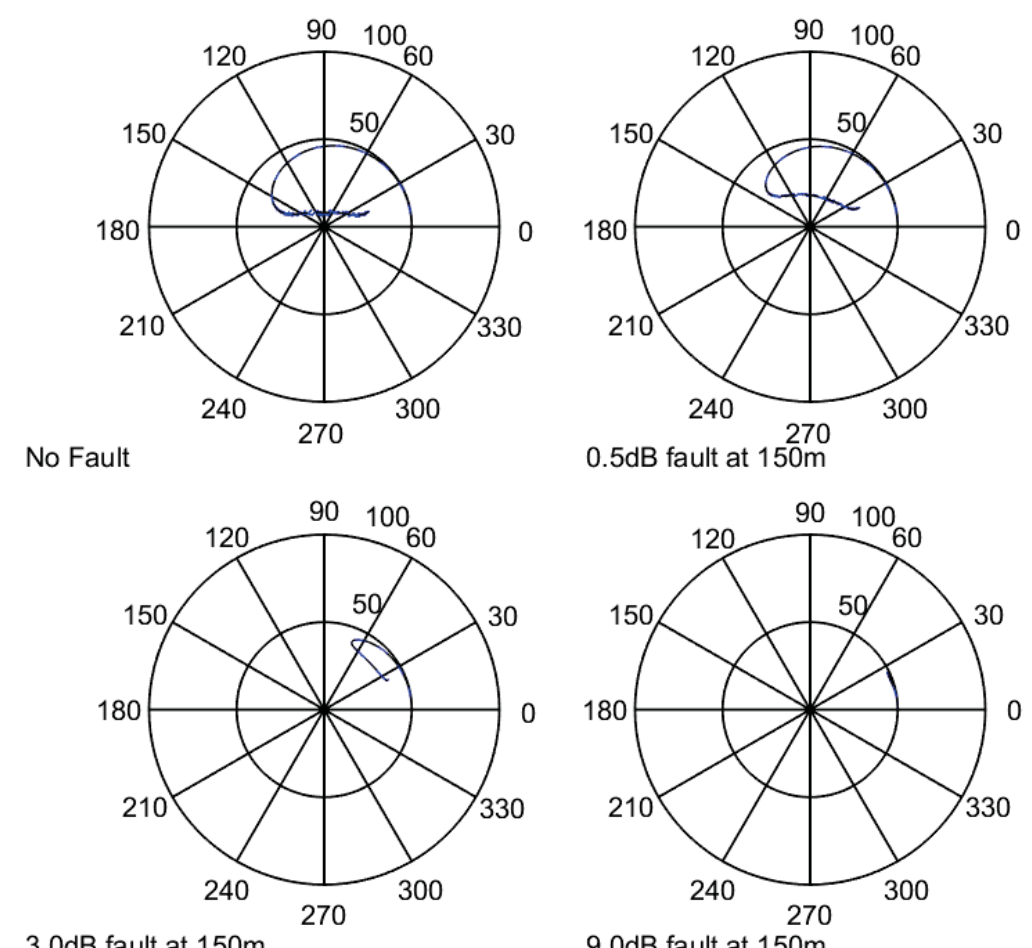

$9.0 \mathrm{~dB}$ fault at $150 \mathrm{~m}$

Figure 6.6: Polar plots for short single fiber link simulation with varying fault intensity $(d=150 m)$

Next, the same short $1.1 \mathrm{~km}$ point to point fiber link simulation is repeated considering this time that a fixed fault intensity of $\delta=3 d B$ occurs at a range of different alternative positions within the fiber. The results are also very promising as can be seen in figure 6.7. They represent the method's ability to estimate a loss anywhere along a short $\sim 1 \mathrm{~km}$ fiber link. Following the linear amplitude and phase versus frequency plot, a set of polar graphs present an alternative view of the results in 6.8. 

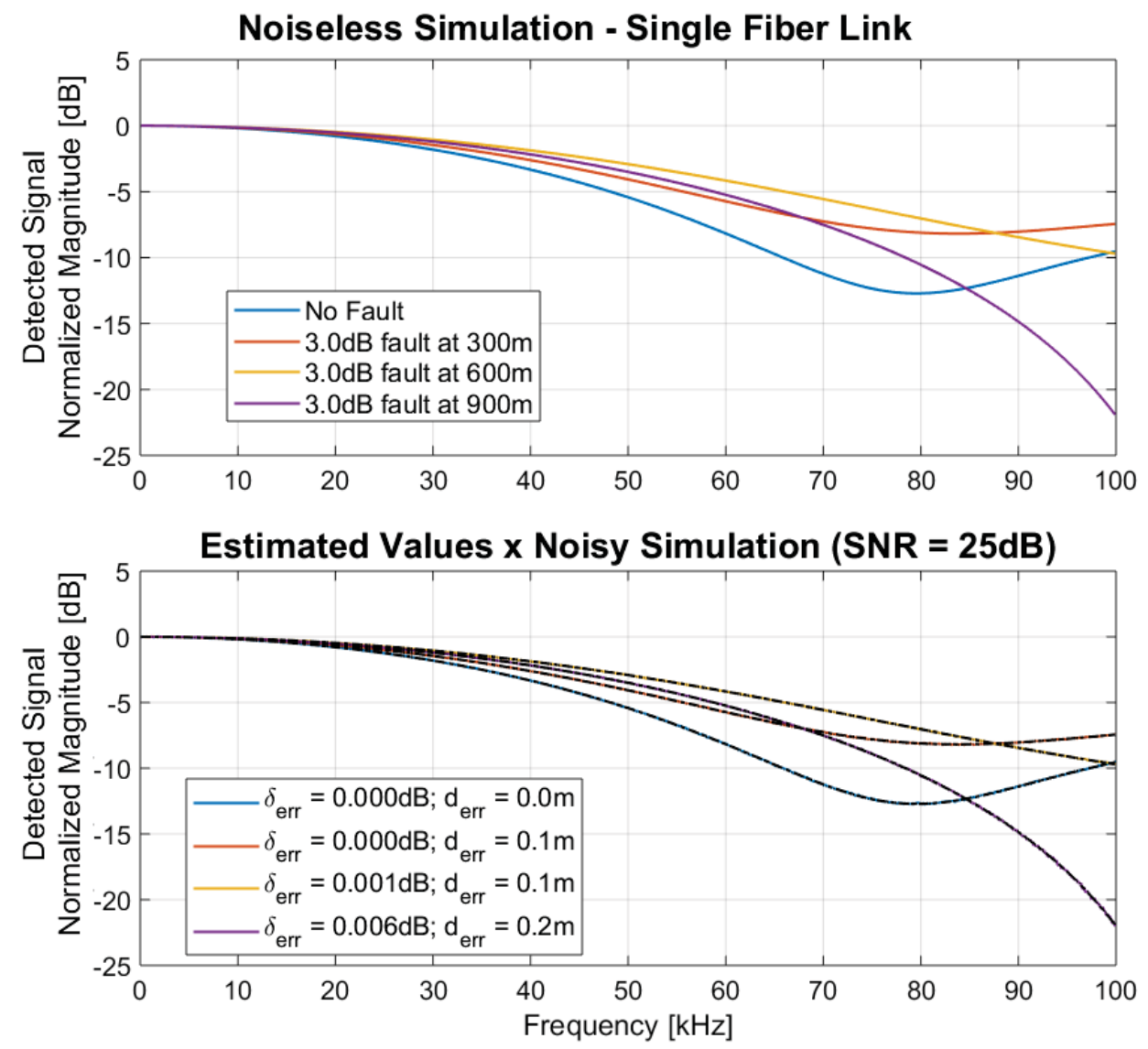

Figure 6.7: Simulation for short single fiber link with varying fault position $(\delta=3 d B)$ 

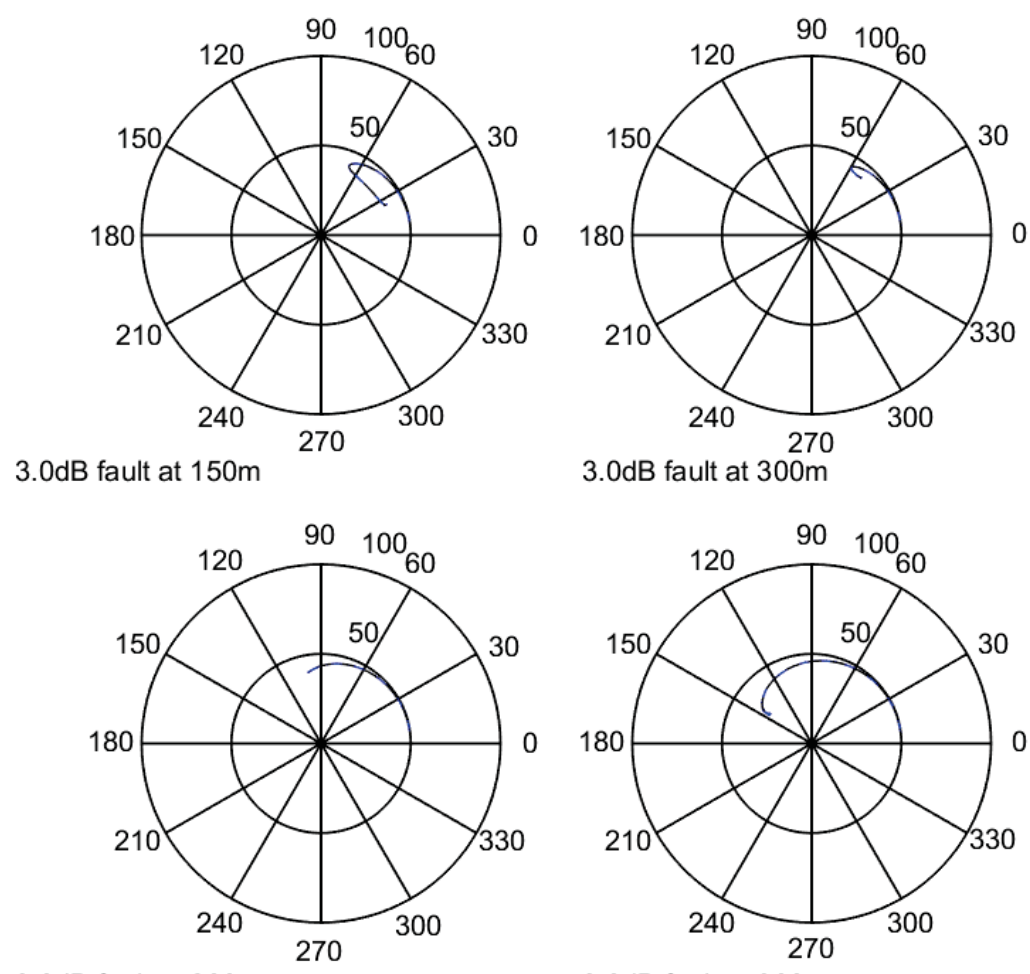

$3.0 \mathrm{~dB}$ fault at $600 \mathrm{~m}$

3.0dB fault at $900 \mathrm{~m}$

Figure 6.8: Polar plots for short single fiber link simulation with varying fault position $(\delta=3 d B)$

The simulation described was repeated 200 times with pseudo-random additive noise generated at each turn. In this manner, some insight could be gathered to the statistical capacity of the proposed fitting algorithm to estimate an unknown fault position and its intensity.

Statistical analysis was obtained for the short $1.1 \mathrm{~km}$ fiber with a set of previously known faults, considering a fixed fault of $3 d B$ intensity occurring at different positions along the fiber. It indicates that the response of the estimator is very precise for most cases, with higher estimation error occurring for faults close to the end of fiber. Figure 6.9 shows estimation within less then $10 \mathrm{~m}$ from simulated fault location and less than $0.1 \mathrm{~dB}$ error in fault intensity estimation. 

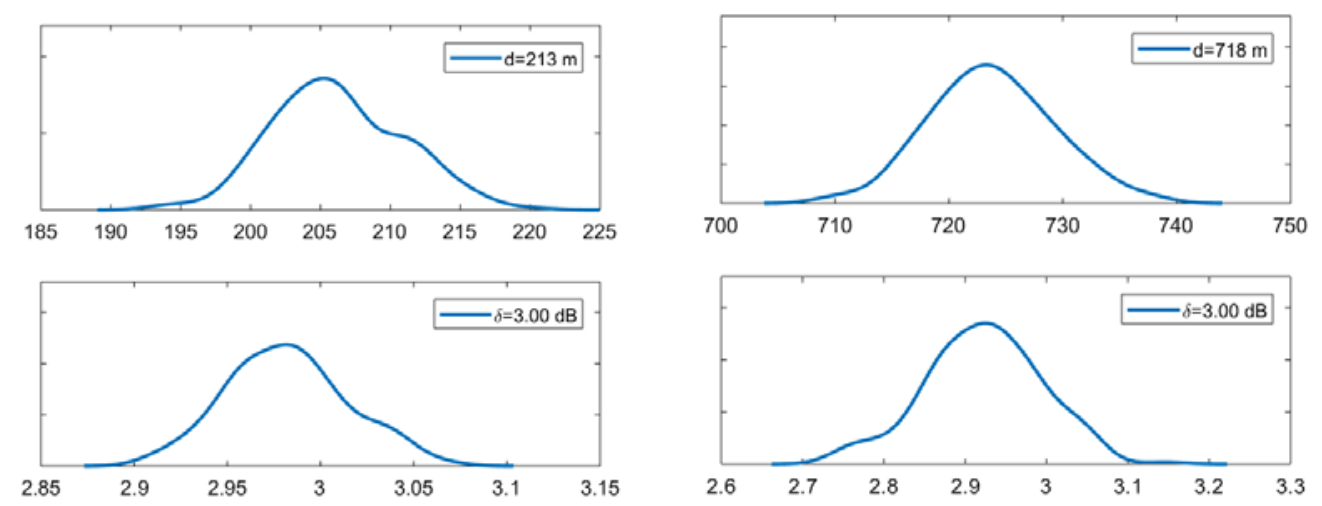

Figure 6.9: Statistical analysis for fault location and intensity estimation in $1.1 \mathrm{~km}$ fiber with some known existing faults.

The graphs in figure 6.10 summarize the obtained statistical results for multiple fault locations in the simulated fiber link. The estimation error is larger at the very beginning of the fiber when the lossy fiber section dominates the result as well as close to the end of the link, which is dominated by the fiber section without loss. The latter presents higher fault estimation error because less power related to the fault is available for comparison. 

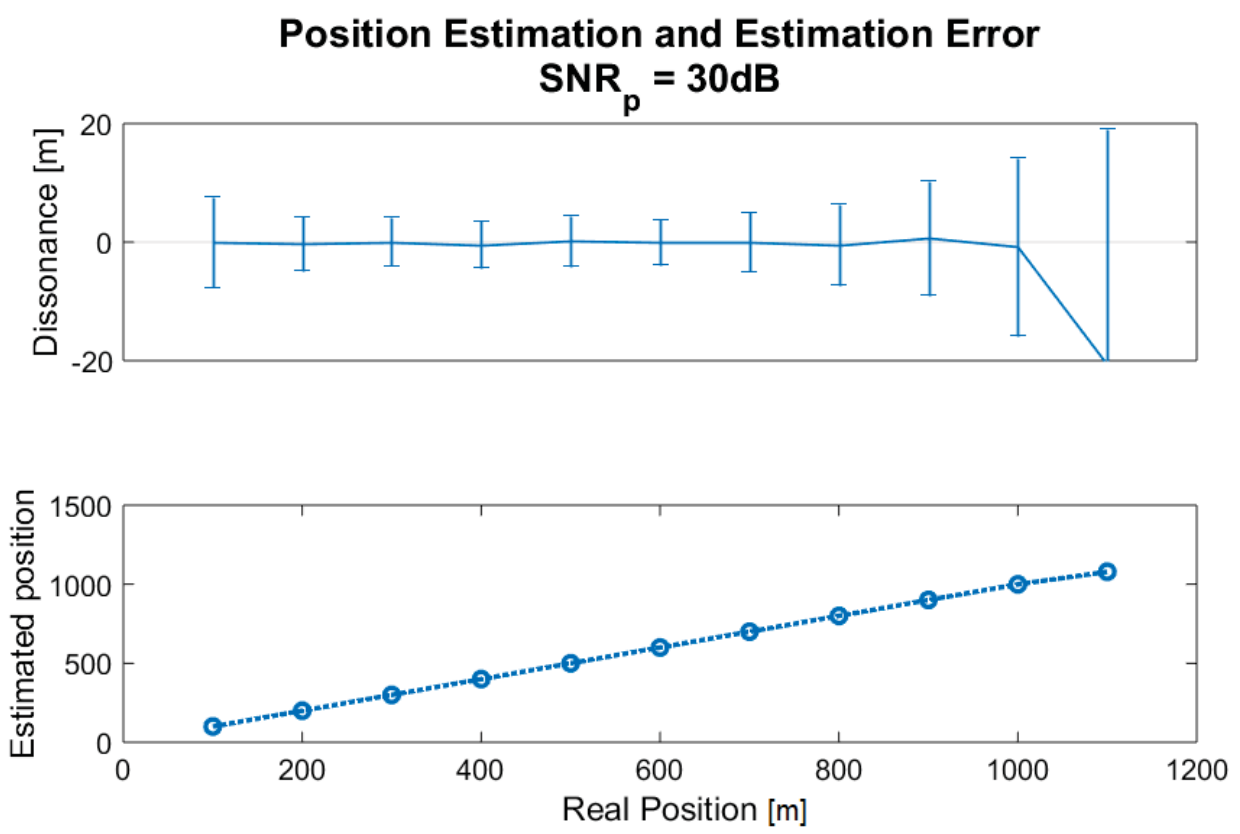

Loss Estimation Error

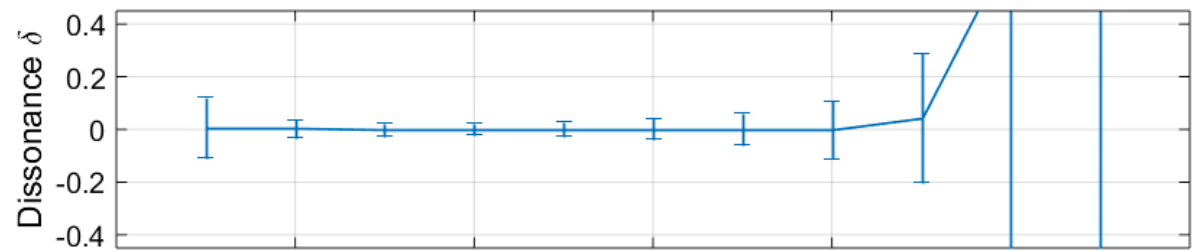

Figure 6.10: Statistical analysis for short single fiber link simulation with varying fault position $(\delta=3 d B)$

Another interesting simulation result is presented in figure 6.11. It represents the case where a short $L=1.1 \mathrm{~km}$ fiber which has a known large loss $(6 d B)$ at a known location $d_{0}=400 \mathrm{~m}$ is tested. An unknown fault then appears at a location further along $\left(d>d_{0}\right)$ in the link. This situation can be easily considered as the existence of a power splitter at a known location when it is desirable to measure faults occurring beyond it. Results from this setting indicate that the solution is likely a good candidate for monitoring TDM-PON networks as will be discussed further on. The simulation output is, once more, very adequate showing estimation within less than $10 \mathrm{~m}$ of the fault location even for a small fault located after a much larger known one. 

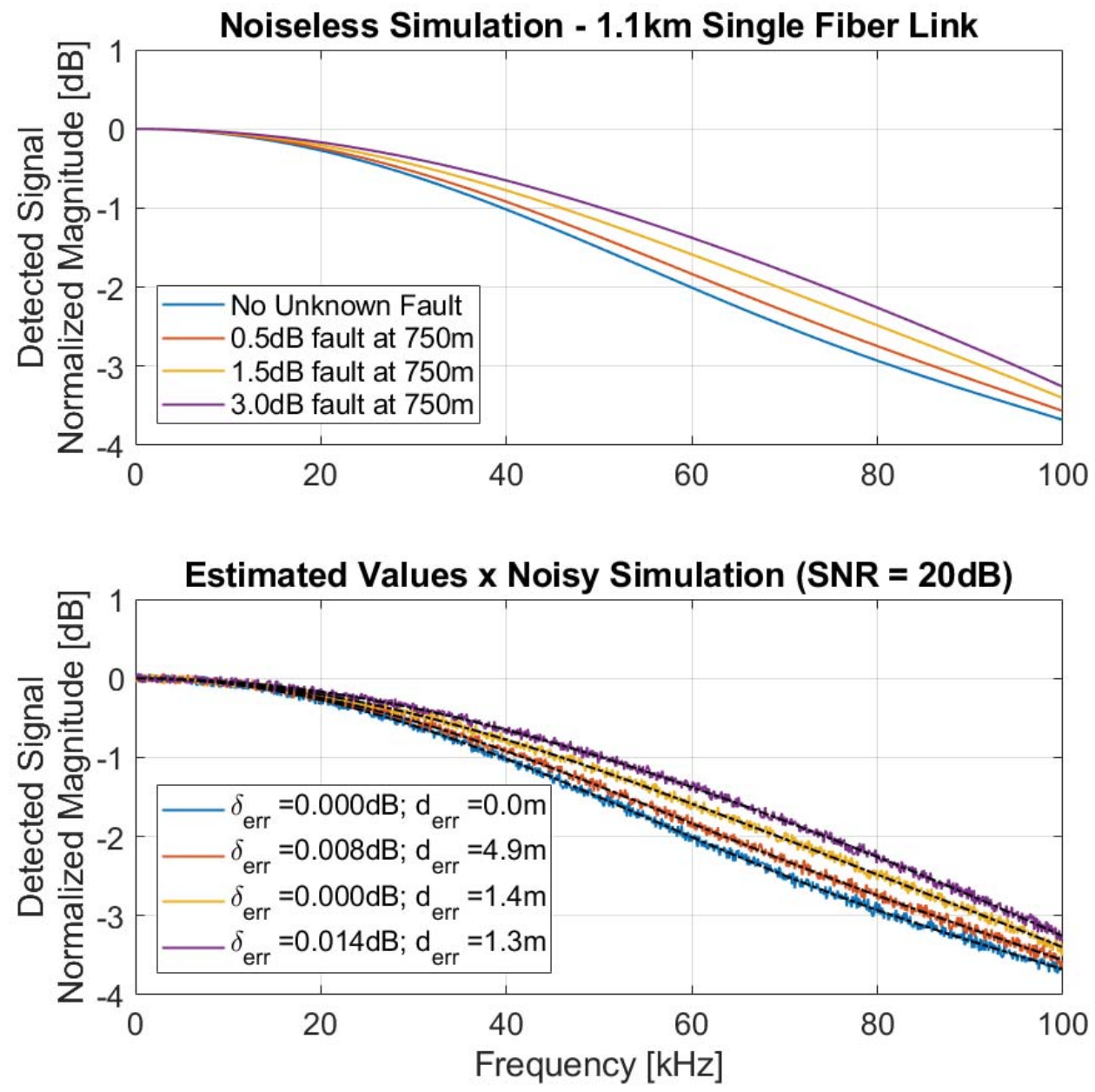

Figure 6.11: Simulation for short single fiber link with a known existing loss and varying fault intensity

Following the same principle, the model for a passive branched network (PON) was used to generate the signal detected. Primarily, the position and intensity of existing faults were considered in a $N \times M$ matrix form and a new fault was represented by a sparse vector $d$ for the position and a sparse vector $\delta$ for the corresponding intensity, both of length $N$, where the only nonzero term in each would correspond to the branch of the PON where the fault occurred.

A simulation following this reasoning is presented here in figure 6.12. It corresponds to the model of a $1: 4$ TDM-PON with $\delta_{s}=0.1 d B$ splitter excess insertion loss and $2 \mathrm{~km}$ feeder fiber. The branches simulated have lengths of $150 \mathrm{~m}, 300 \mathrm{~m}, 450 \mathrm{~m}$ and $500 \mathrm{~m}$, where the position of the end user in each branch $L_{n}$ are given by adding $L_{0}$, as $2150 \mathrm{~m}, 2300 \mathrm{~m}, 2450 \mathrm{~m}$ and $2500 \mathrm{~m}$. The result corresponds to the estimation of a $3 d B$ fault at a distance $2250 \mathrm{~m}$ from 
the central office occurring in the branch with length $L_{i}=2450 \mathrm{~m}$ from the input to the feeder fiber.

The simulation output shown in figure 6.12 considers a scenario where very high signal to noise ratio $(\mathrm{SNR})$ of $60 \mathrm{~dB}$ relative to $P_{0}$ can be obtained providing excellent accuracy and an alternative simulation of a more realistic SNR situation of $25 d B$ relative to $P_{0}$, comparable to that used in the pointto-point simulations, which was effectively measured for the single fiber link experiments.
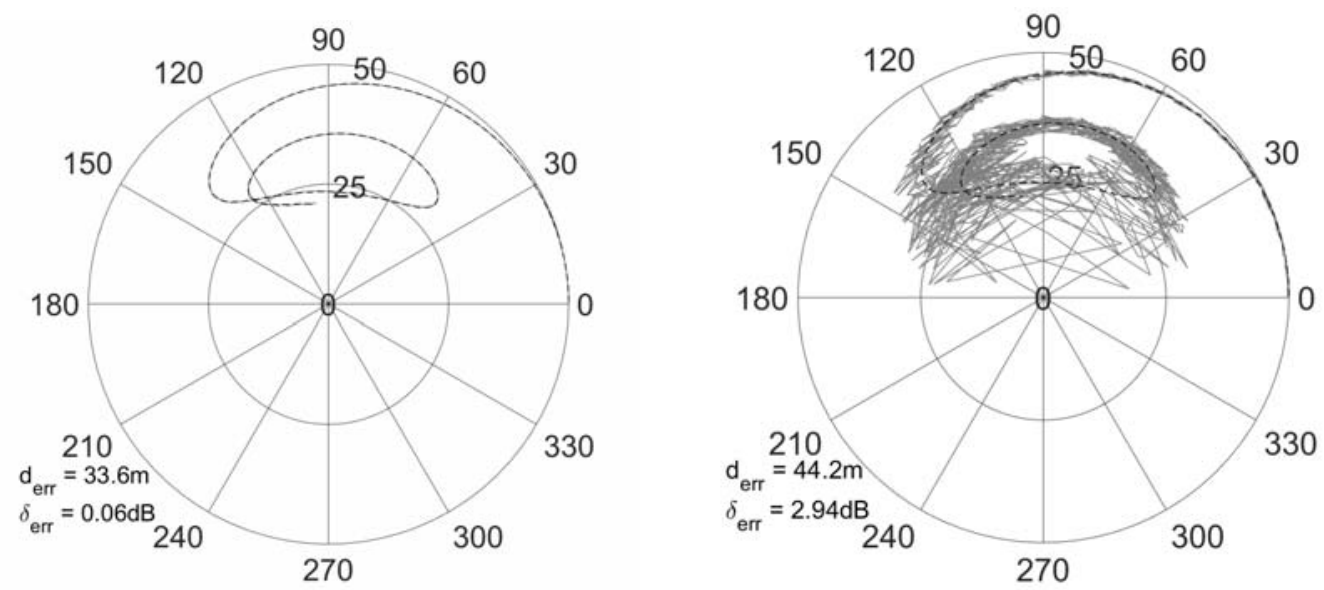

Figure 6.12: Simulation for a $1: 4$ PON with a $3 d B$ fault at one branch with high SNR and realistic SNR.

The error between simulated fault location and intensity is beyond acceptable in this case, for realistic SNR values, with fault location error greater that $40 \mathrm{~m}$ and intensity estimation error greater than $2.5 \mathrm{~dB}$. Furthermore, repetition of the simulation does not converge to the same values, sometimes leading to wrongly estimating the faulty branch.

Therefore, another approach to the optimization goal function is clearly necessary in order to provide reasonable estimation values. The results shown above correspond to an attempt to apply the method employed with equation 5-11 using equation 5-24 instead, which lead to a multi-variate optimization problem with $2 N$ degrees of freedom, where the algorithm tries to estimate a possible new fault position and its intensity occurring in any of the $N$ branches simultaneously. This solution doesn't always converge within a reasonable (or even large) number of steps, thus leading to poor resolution. 


\section{2}

\section{Simulations using the Differential Mathematical Model}

A new method is proposed considering a variation in the mathematical model which may be represented in a differential form considering a reference measurement $S_{0}(f)$ of the detected signal for the system under test is available, evaluating the difference between the observed data with the unknown fault and the model prior to that fault (with previously known faults only). This solution based on $\Delta S(f)=S_{0}(f)-S(f)$ was considered for both point-to point and point-to-multi-point (TDM-PON) links yielding very good simulation results. The outcome for a variety of length and fault combinations are summarized subsequently.

Point-to-point links of different lengths were simulated repeatedly using the differential model previously presented with various combinations of fault position and intensity. Common parameters used in the simulation were as follows: average attenuation in the fiber $\alpha=0.24 \mathrm{~dB} / \mathrm{km}$; average group refraction index $n_{g}=1.4682$; frequency sweep range from $50 \mathrm{~Hz}$ to $100 \mathrm{kHz}$ in steps of $50 \mathrm{~Hz}$; normalized amplitude $A_{0}=1$. Random white complex Gaussian noise (30dB below the maximum power detected) was added in each simulation and the curve fitting algorithm was used to estimate the fault parameters. Again the noise power was chosen to imitate the results observed in experimental trials.

A $12 \mathrm{~km}$ fiber was simulated using the new proposed mathematical model for estimation with a fault occurring $8 \mathrm{~km}$ away from the input with different intensities. The outcome of these simulations indicate that this differential solution is more precise in determining the fault parameters of interest. Furthermore, analyzing the statistical results shown in figure 6.13, there's an indication that larger faults are easier to locate, and thus correspond to a smaller variation in the position estimation. Still, all simulated results present high accuracy with location error up to a few meters on average.

Other fiber lengths and fault locations were considered yielding similar results. Table 6.1 shows the average estimation error for each parameter after 200 repeated simulations. Results show convergence of the estimation into the desired values which were set in each simulation by design within tolerable ranges for fault estimation techniques.

In order to evaluate the variation of fault intensity at different positions, a link of $1.1 \mathrm{~km}$ was simulated again with $3 d B$ faults located at different positions, this time with the differential model as fitting parameter. Comparison with the simulated results using the complete mathematical description of the detected signal shows this solution to be more precise, as can be noted from 

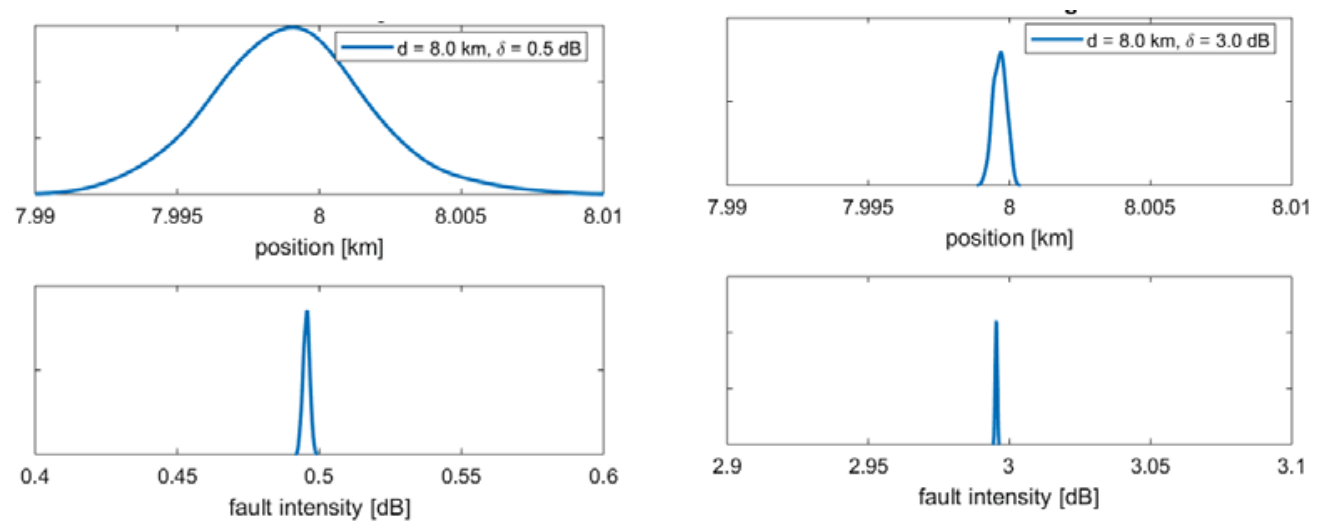

Figure 6.13: Statistical analysis for the simulation of a $12 \mathrm{~km}$ link with a fault occurring $8 \mathrm{~km}$ away from the input using the differential model for estimation.

Table 6.1: Point-to-point link simulation with Differential Estimation

\begin{tabular}{|c|c|c|c|c|}
\hline $\begin{array}{c}\text { Link } \\
\text { Length }\end{array}$ & d & $\delta$ & $\begin{array}{l}\text { Average Error } \\
\left(\mathrm{d}_{\mathrm{err}}[\mathbf{m}]\right)\end{array}$ & $\begin{array}{c}\text { Average Error } \\
\left(\delta_{\text {err }}[\mathbf{d B}]\right)\end{array}$ \\
\hline \multirow{8}{*}{$\begin{array}{l}\text { Long Link } \\
\quad(12 \mathrm{~km})\end{array}$} & \multirow{4}{*}{$4 k m$} & $0.5 d B$ & 3.04 & 0.0020 \\
\hline & & $1.5 d B$ & 1.22 & 0.0020 \\
\hline & & $3.0 d B$ & 0.81 & 0.0020 \\
\hline & & $5.0 d B$ & 0.69 & 0.0020 \\
\hline & \multirow{4}{*}{$8 k m$} & $0.5 d B$ & 2.25 & 0.0045 \\
\hline & & $1.5 d B$ & 0.73 & 0.0046 \\
\hline & & $3.0 d B$ & 0.35 & 0.0046 \\
\hline & & $5.0 d B$ & 0.27 & 0.0046 \\
\hline \multirow{16}{*}{$\begin{array}{l}\text { Short Link } \\
\quad(2 \mathrm{~km})\end{array}$} & \multirow{4}{*}{$800 m$} & $0.5 d B$ & 3.45 & 0.0014 \\
\hline & & $1.5 d B$ & 1.46 & 0.0014 \\
\hline & & $3.0 d B$ & 0.97 & 0.0014 \\
\hline & & $5.0 d B$ & 0.80 & 0.0013 \\
\hline & \multirow{4}{*}{$900 m$} & $0.5 d B$ & 3.08 & 0.0017 \\
\hline & & $1.5 d B$ & 1.28 & 0.0016 \\
\hline & & $3.0 d B$ & 0.85 & 0.0016 \\
\hline & & $5.0 d B$ & 0.71 & 0.0016 \\
\hline & \multirow{4}{*}{$1.1 \mathrm{~km}$} & $0.5 d B$ & 1.50 & 0.0019 \\
\hline & & $1.5 d B$ & 0.61 & 0.0019 \\
\hline & & $3.0 d B$ & 0.37 & 0.0018 \\
\hline & & $5.0 d B$ & 0.30 & 0.0018 \\
\hline & \multirow{4}{*}{$1.2 \mathrm{~km}$} & $0.5 d B$ & 1.24 & 0.0019 \\
\hline & & $1.5 d B$ & 0.35 & 0.0019 \\
\hline & & $3.0 d B$ & 0.17 & 0.0019 \\
\hline & & $5.0 d B$ & 0.14 & 0.0019 \\
\hline
\end{tabular}

figure 6.14. The outcome for simulation of multiple positions is shown in table 6.2. Noticeably, the estimation error is greater at fault positions close to the 
end of the fiber in good agreement with the technique using the direct mathematical model. This result is derived because the energy contribution from the faulty fiber section is much smaller than the energy from the undisturbed section.

Again, the estimation error is tolerable for location of the fault and very precise for intensity measurements, comparable to commercial OTDR results. In this trial, again faults closer to the end of fiber are more troublesome to determine. Simulations considering noisier signals were also performed and convergence to the correct values is maintained, although the average error was considerably higher, especially in the extremes. Nonetheless, the estimation error found in those cases was still tolerable, averaging within a few tens of meters in the extreme cases (closer to the fiber end). The results presented in this work correspond to SNR similar to the experimentally observed values.
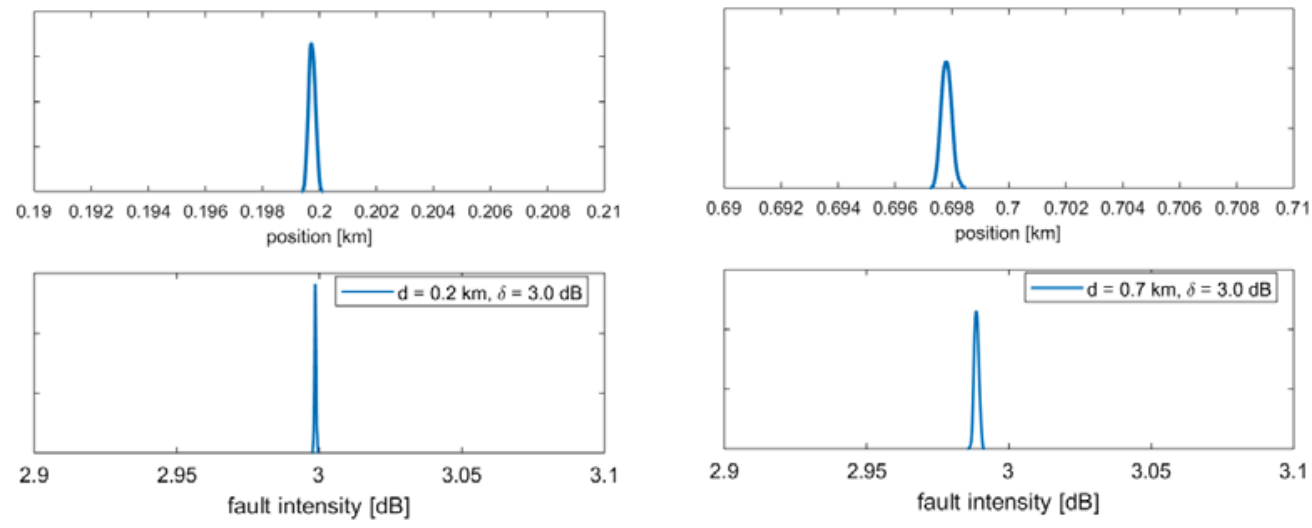

Figure 6.14: Statistical analysis for the simulation of a $1.1 \mathrm{~km}$ link with a $3 d B$ fault occurring at different locations, using the differential model for estimation.

Table 6.2: Simulation with Differential Estimation for various positions

\begin{tabular}{|c|c|c|c|c|}
\hline $\begin{array}{c}\text { Link } \\
\text { Length }\end{array}$ & d & $\delta$ & $\begin{array}{l}\text { Average Error } \\
\left(\mathrm{d}_{\mathrm{err}}[\mathbf{m}]\right)\end{array}$ & $\begin{array}{l}\text { Average Error } \\
\left(\delta_{\text {err }}[\mathbf{d B}]\right)\end{array}$ \\
\hline \multirow{10}{*}{$\begin{array}{l}\text { Short Link } \\
(1.1 \mathrm{~km})\end{array}$} & $100 m$ & \multirow{10}{*}{$3 d B$} & 0.40 & 0.0013 \\
\hline & $200 m$ & & 0.25 & 0.0014 \\
\hline & $300 m$ & & 0.21 & 0.0015 \\
\hline & $400 m$ & & 0.23 & 0.0018 \\
\hline & $500 m$ & & 0.41 & 0.0026 \\
\hline & $600 m$ & & 0.91 & 0.0048 \\
\hline & $700 m$ & & 2.19 & 0.0113 \\
\hline & $800 m$ & & 5.66 & 0.0347 \\
\hline & $900 m$ & & 17.16 & 0.1430 \\
\hline & $1000 m$ & & 54.54 & 0.6977 \\
\hline
\end{tabular}



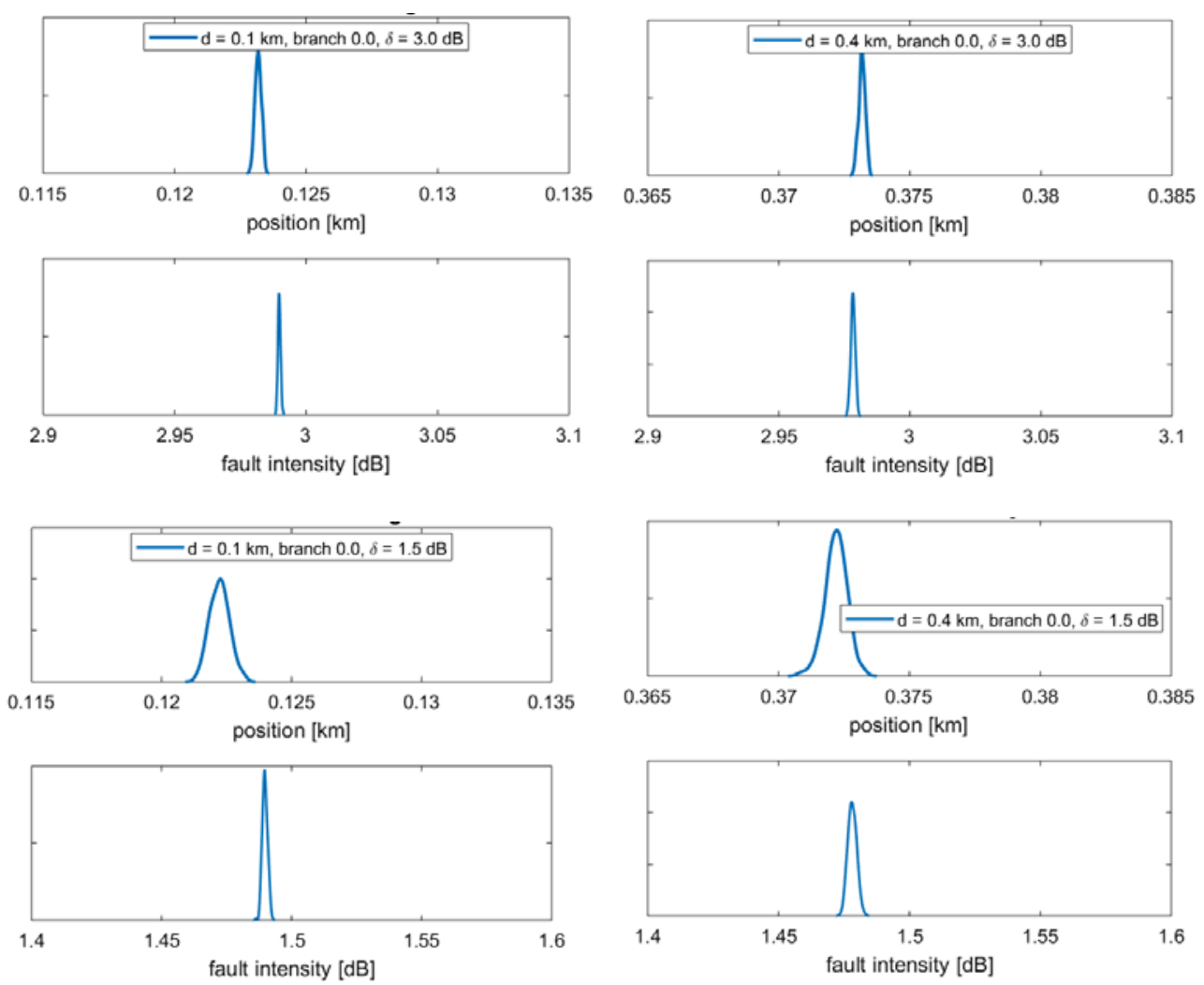

Figure 6.15: Statistical analysis for the simulation of a PON link with faults occurring in the feeder fiber, using the differential model for estimation.

As mentioned, one important innovation of the proposed method is that it may be adapted to monitoring of TDM-PONs. A branched network was thus simulated with the estimation for fault occurrence based on the differential model discussed in the previous chapter. In this case, the optimization algorithm runs separately considering the fault to be either in the feeder or in each of the $N$ branches and selects the result containing the lowest residual value for the minimization as the true estimation for the location. Multiple combinations of fault position $d$ (distance from the $\mathrm{CO}$ ), intensity $\delta$ and branch were considered in the simulation. The same common parameters used for simulation of point-to-point link were chosen.

The TDM-PON simulated was defined as having feeder line of length $L_{0}=500 \mathrm{~m}$, followed by a $1: 4$ splitter incurring and additional insertion loss of $\delta_{s}=0.5 \mathrm{~dB}$ and branches ending in lengths $L_{1}=1 \mathrm{~km}, L_{2}=1.5 \mathrm{~km}$, $L_{3}=2 \mathrm{~km}, L_{4}=2.5 \mathrm{~km}$. Statistical results for faults occurring in the feeder line shown in 6.15 are in good agreement with the point-to-point case, as expected. The method underestimates the fault position slightly (less then $5 \mathrm{~m}$ on average).

The case where faults occurred at different positions in each of the four branches were also considered. Figure 6.16 shows some examples of 
data obtained from simulation. Noticeably, faults close to the end of the corresponding branch fiber resulted in less accurate location. A summary of all the results evaluated is presented in table 6.3. Again, the error between estimated and expected values of the fault parameters present outstanding precision. Poorer SNR leads to poorer results in this case also, but still within reasonable error margins.

Table 6.3: PON link simulation with Differential Estimation

\begin{tabular}{|c|c|c|c|c|}
\hline $\begin{array}{c}\text { Fault } \\
\text { Location }\end{array}$ & $\mathrm{d}$ & $\delta$ & $\begin{array}{l}\text { Average Error } \\
\left(\mathrm{d}_{\mathrm{err}}[\mathbf{m}]\right)\end{array}$ & $\begin{array}{c}\text { Average Error } \\
\left(\delta_{\text {err }}[\mathbf{d B}]\right)\end{array}$ \\
\hline \multirow{6}{*}{$\begin{array}{c}\text { Feeder } \\
\left(L_{0}=500 m\right)\end{array}$} & \multirow{2}{*}{$125 m$} & $1.5 d B$ & 2.77 & 0.0102 \\
\hline & & $3.0 d B$ & 1.82 & 0.0102 \\
\hline & \multirow{2}{*}{$250 m$} & $1.5 \mathrm{~dB}$ & 3.14 & 0.0155 \\
\hline & & $3.0 d B$ & 2.09 & 0.0155 \\
\hline & \multirow{2}{*}{$375 m$} & $1.5 \mathrm{~dB}$ & 2.80 & 0.0218 \\
\hline & & $3.0 d B$ & 1.82 & 0.0217 \\
\hline \multirow{2}{*}{$\begin{array}{c}1^{s t} \text { Branch } \\
\left(L_{1}=1 k m\right)\end{array}$} & \multirow{2}{*}{$800 m$} & $1.5 d B$ & 13.95 & 0.0635 \\
\hline & & $3.0 d B$ & 4.40 & 0.0332 \\
\hline \multirow{2}{*}{$\begin{array}{c}2^{\text {nd }} \text { Branch } \\
\left(L_{2}=1.5 \mathrm{~km}\right)\end{array}$} & \multirow{2}{*}{$800 m$} & $1.5 d B$ & 5.28 & 0.0092 \\
\hline & & $3.0 d B$ & 1.84 & 0.0042 \\
\hline \multirow{4}{*}{$\begin{array}{l}3^{r d} \text { Branch } \\
\left(L_{3}=2 k m\right)\end{array}$} & \multirow{2}{*}{$800 m$} & $1.5 \mathrm{~dB}$ & 4.74 & 0.0076 \\
\hline & & $3.0 d B$ & 1.68 & 0.0034 \\
\hline & \multirow{2}{*}{$1.6 \mathrm{~km}$} & $1.5 d B$ & 9.44 & 0.0299 \\
\hline & & $3.0 d B$ & 3.13 & 0.0153 \\
\hline \multirow{4}{*}{$\begin{array}{c}4^{\text {th }} \text { Branch } \\
\left(L_{4}=2.5 \mathrm{~km}\right)\end{array}$} & \multirow{2}{*}{$800 m$} & $1.5 d B$ & 4.86 & 0.0083 \\
\hline & & $3.0 d B$ & 1.82 & 0.0043 \\
\hline & \multirow{2}{*}{$1.6 \mathrm{~km}$} & $1.5 d B$ & 4.24 & 0.0079 \\
\hline & & $3.0 d B$ & 1.46 & 0.0037 \\
\hline
\end{tabular}

Scenarios with existing faults were not simulated. As discussed in Appendix A, they can be treated in a similar fashion to the procedure used for estimating in which branch of the PON a new fault occurs. That is, the appropriate modification is done to the goal function in the optimization (mathematical model) for each possible scenario of a new fault occurring. Assuming a finite number of previously known faults $M$, the optimization must consider $M+1$ possible alternatives for the position $\left(d<d_{1}, d_{1}<d<d_{2}, \ldots d_{M-1}<d<\right.$ $\left.d_{M}, d_{M}<d\right)$, estimate the fault parameters considering each to be true, then select the one which corresponds to the least residual of the minimization. The alternative optimization settings may run concurrently in parallel, but their programming is slightly more complex that the single fault case.

Simulation results indicate that the proposed methods may estimate 

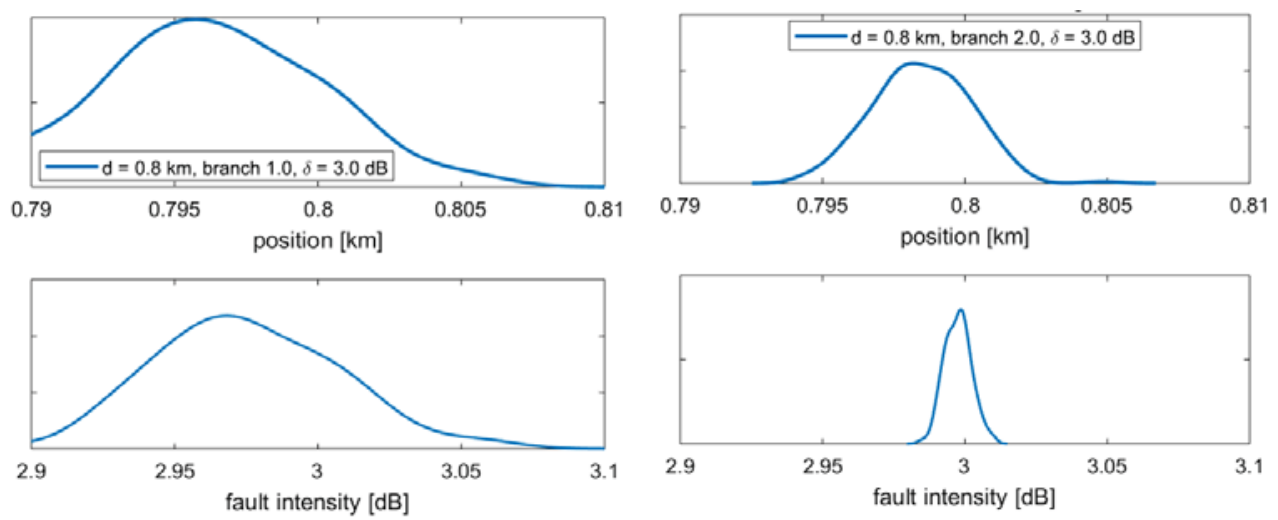

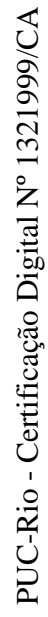
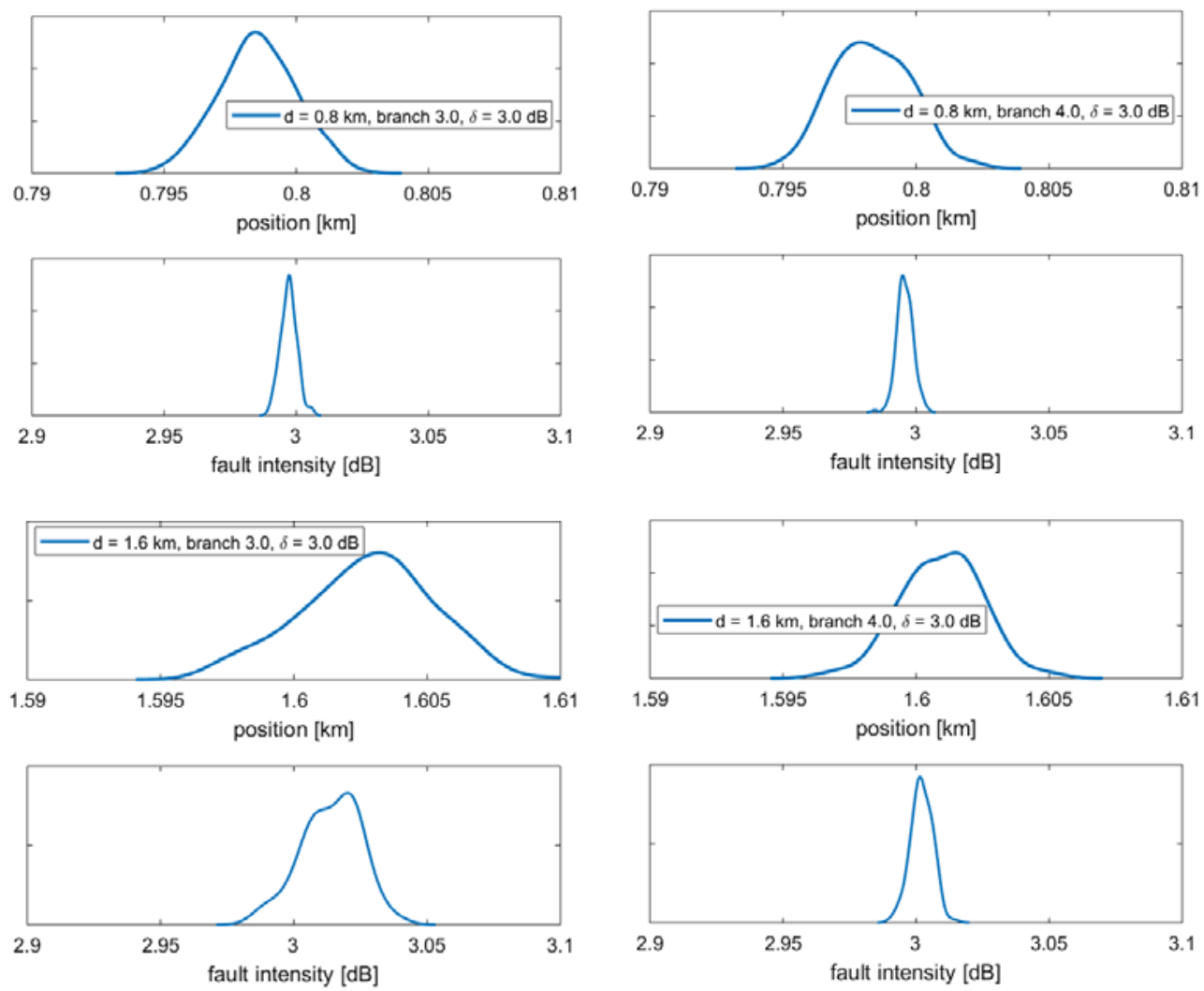

Figure 6.16: Statistical analysis for the simulation of a PON link with a $3 d B$ fault occurring in the various locations in different branches, using the differential model for estimation. 
the occurrence of a new fault in a fiber link with sufficiently high precision. Furthermore, statistical analysis show that the differential method is usually more accurate than optimization using the complete mathematical model. This outcome is even more evident for the case of branched networks, where optimization with the complete description of the detected signal as the goal function often did not converge.

The encouraging outcome of the simulations produced must be confirmed by an experimental setting. The experiments that were realized with single mode fiber setup as the proposed links in a laboratory environment for within the ambit of this study are presented next. 


\section{Experimental Results}

A set of experiments were programmed and performed in a controlled laboratory setting in order to validate the theoretical models proposed and the simulation results. The mathematical equations developed to be used in the fiber monitoring solution presented in this thesis approximate the detected signal resulting from Rayleigh backscatter of the modulated optical input through either a point-to-point link or an optical fiber network (PON) directly or relative to a prior state. The effects of the measuring equipment, namely the laser diode, the photodetector and the electronic amplifier, must be taken into account or removed from the experimental results as mentioned before.

A measurement was, therefore, taken for the setup represented in 4.3, without any fiber. A break at port 2 of the circulator was obtained adding a short piece of fiber at this point, which gives a reflection providing enough optical power for the detector to get good measurements, but doesn't affect the phase of the reference. Hence, sweeping the frequency with a reflection at port 2 of the circulator in the manner described, allowed for the system setup representation. This measurement represents the transfer function of the setup equipment, or setup characterization reference measurement, and was incorporated in the fiber response model as described by 7-1.

$$
S_{\text {experimental }}(f)=S_{\text {model }}(f) H_{\text {equipment }}(f)
$$

The fitting algorithm attempts to match the data extracted from the Lock-in Amplifier using the technique described with the selected mathematical model in each method to the expected experimental signal curve $S_{\text {experimental }}(f)$. The output of the algorithm provides estimated values of fault position and loss factor intensity given the previously known state of the fiber (e.g. existing faults due to connectors). The algorithm compares the experimental data to the modeled curve which includes information about position and loss factor intensities of known existing faults (previous state) in a way that only one unknown (new) fault is detected. 


\section{1}

\section{Coherent Rayleigh Noise}

During the experimental trials of the setup described in the previous section, an unforeseen noisy effect fading slowly as the frequency changed was noted. This effect was not modeled in the mathematical description of the detected signal which is based in physical properties of the Rayleigh backscatter in the fiber. This additional noise is associated with Coherent Rayleigh Noise (CRN). The noise was identified in the amplitude and phase measurements after a change of laser source was made and was treated for the experiments realized afterwards. Nonetheless, experimental data indicate its presence in the initial tests before the exchange as well, where the mitigation solution which will be described next was not employed.

CRN results from interferometric conversion of laser phase noise to intensity noise due to either multiple reflections along the fiber or coherent interference of the Rayleigh backscattered light with light in the fiber traveling in the same direction through different lengths $[86,87]$.

In this setup, reflections were suppressed as much as possible such that CRN was caused by the interference between light backscattered from different parts of the fiber within the coherence length of the laser. Since CRN affects mainly the lower frequencies of the spectrum, it is very damaging to the proposed low frequency sweep solution and may result in wrongly estimating the location and intensity of faults.

As suggested in [86], the harming effect of CRN in signal-to-noise ratio can be improved by expanding the linewidth of the laser. This can be done by adding a dithering signal to the laser bias. Enlarging the laser linewidth reduces its coherence length, thus lessening the noise effect. Optical chirping using a saw tooth kind of dithering signal presents the best CRN reduction results because it provides an equally distributed excursion of the wavelength around the optical carrier. In order to compensate for CRN in the proposed solution, some modifications were made to the setup presented earlier.

CRN noise was treated in experiments using a Mitsubishi laser, with large linear region of operation as will be described later on in the Experimental Results chapter. The laser bias current was set to $70.8 \mathrm{~mA}$, equivalent to about $10 \mathrm{dBm}$ optical power at the input to the fiber under test (port 2 of the circulator) and sufficiently far away from its threshold current of $\sim 15 \mathrm{~mA}$. The modulating signal generated by the Lock-in Amplifier was a frequency swept sinusoidal function with amplitude $700 m V_{r m s}$, corresponding to approximately $2 \mathrm{~V}$ peak-to-peak or approximately $40 \mathrm{~mA}$ excursion. This signal was added directly to the bias current as a varying amplitude modulating scheme, so it 
shifted between $50 \mathrm{~mA}$ and $90 \mathrm{~mA}$ as the modulating signal amplitude varied. Due to the linearity of the laser response in this region, the optical power shifted proportionally.

A low power triangular wave signal was chosen to create a dithering signal to be added to the bias current. The wave frequency was set to $2.096 \mathrm{~Hz}$, enough to widen the laser linewidth. Its peak-to-peak amplitude was set to $200 \mathrm{mV}$, shifting the driving current further slightly (approximately $2 \mathrm{~mA}$ ) in each direction, corresponding to about $10 \%$ of the total modulation current. The choice of frequency was ajusted to find the best reduction on the impact of CRN, with care that each sample acquisition included all wavelengths included in the excursion of the chirping signal.

While the matter of CRN was mitigated by this solution, it caused a small periodic amplitude variation to the modulated signal which affected the measurements adding to the amplitude at some values of the frequency sweep and subtracting from it in other values. In order to overcome this problem, a set of 10 samples were acquired from the Lock-in Amplifier for each frequency evaluated in the sweep and the measured values were averaged, thus eliminating the added varying amplitude periodic signal. The choice of the number of samples in the average was such that each measurement from the Lock-in amplifier lasted through a whole period of the added low power signal.

Figure 7.1 shows the frequency swept detected signal measured for a $3 \mathrm{~km}$ fiber link without the CRN mitigation, with the mitigating dithering signal and with both dithering signal and averaging. It is clear that CRN causes the proposed method to wrongly locate faults and determine their intensity, thus reducing its effects leads to better results.

After the discussed changes were made to the setup, SNR of the acquired signal became much higher, leading to much more precise fault estimation measurements. The trade off necessary, other than the added signal power, was a longer acquisition time for the whole sweep. In the experiments presented in this work, after the CRN was treated, measurement time was about $8 \mathrm{~min}$ for a $100 \mathrm{~Hz}-100 \mathrm{kHz}$ sweep, compared to less that $4 \mathrm{~min}$ without the additional steps required for CRN reduction. This result is comparable to commercial OTDR measurement time under similar resolution requirements. The complete setup including these modifications is presented in figure 7.2. 


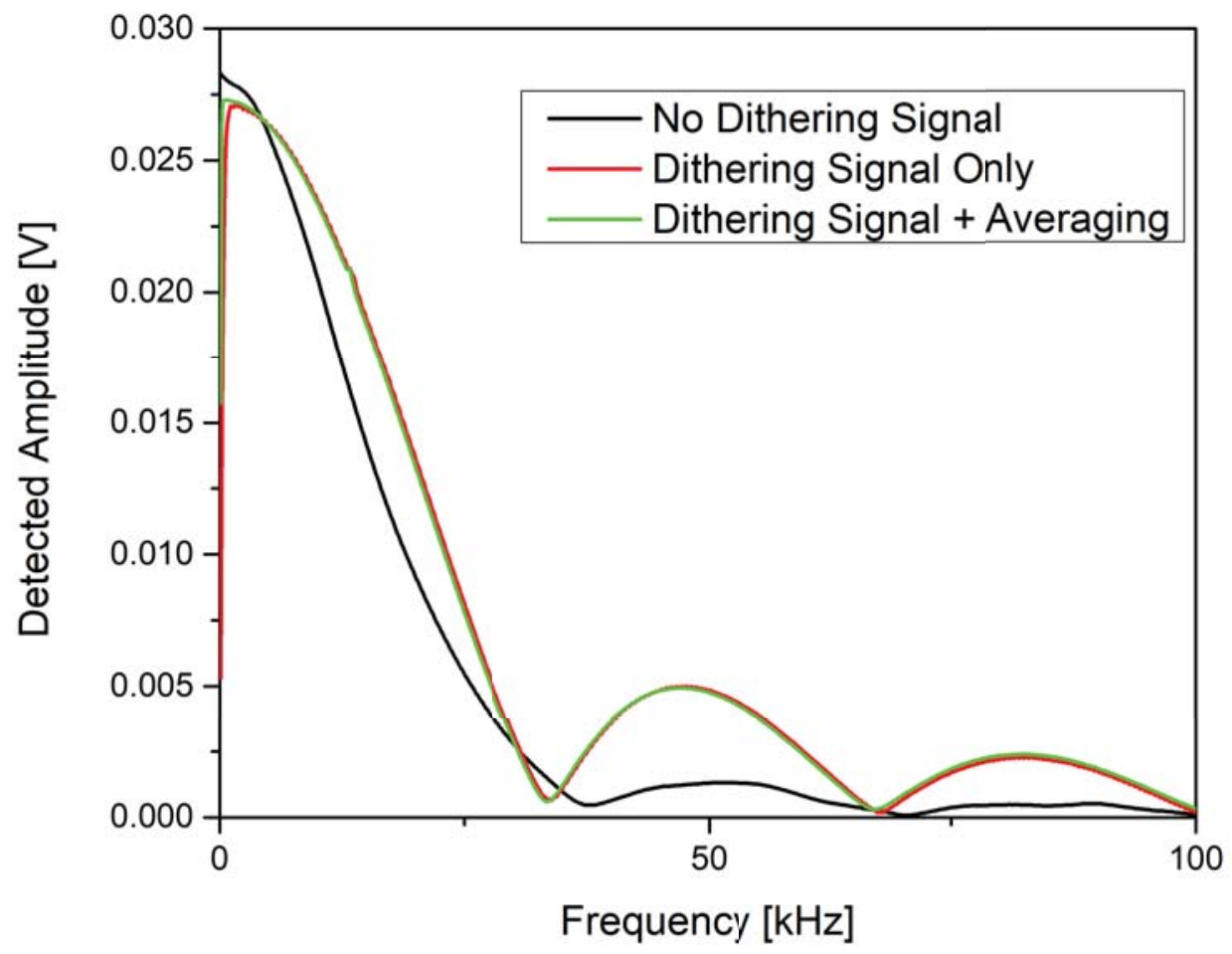

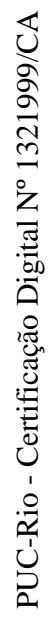

Figure 7.1: Effect of CRN mitigation after adding triangular wave signal and averaging.

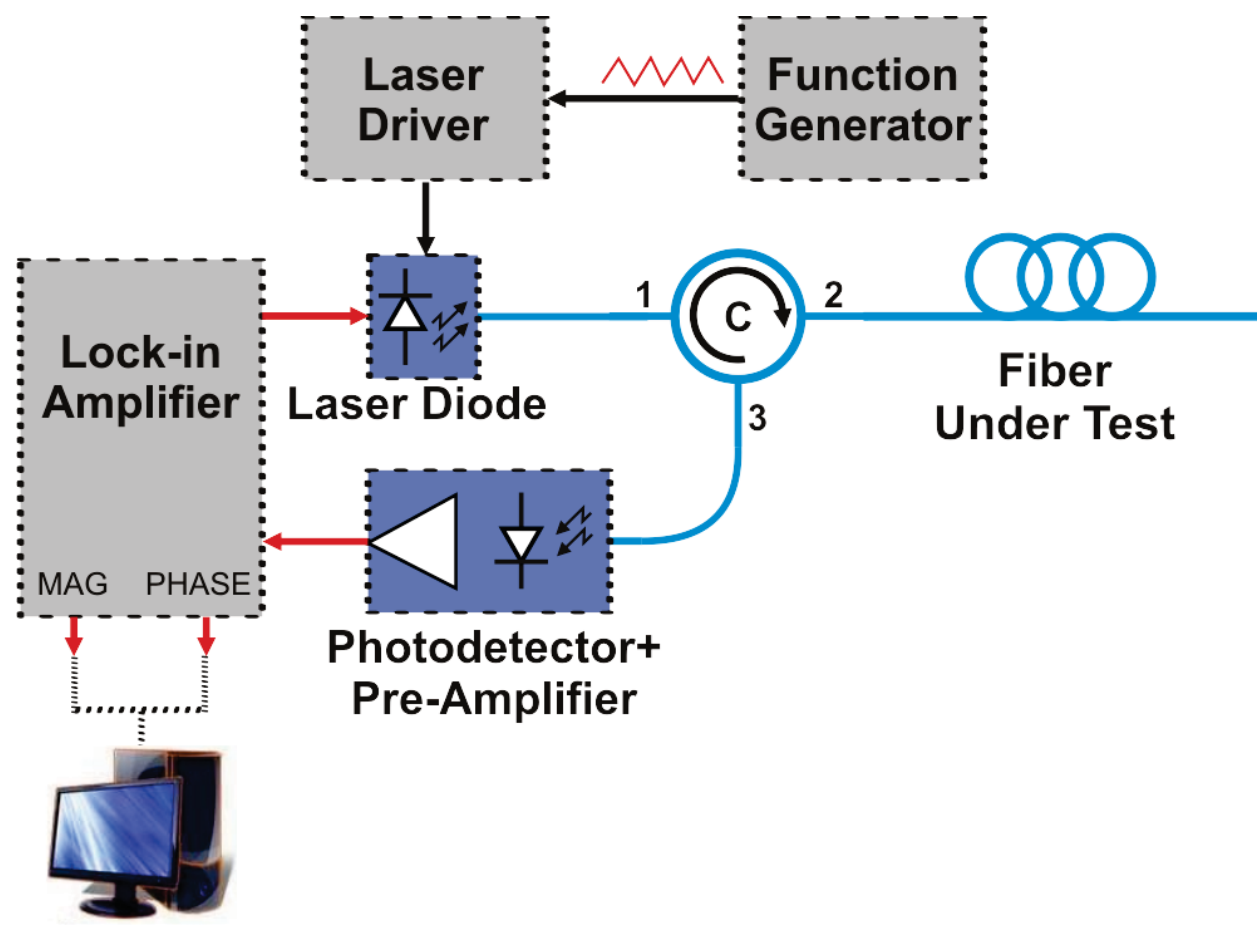

Figure 7.2: Modified experimental setup, including necessary equipment used to reduce the effects of CRN 


\section{2}

\section{Experiments with Complete Mathematical Model for Estimation}

At first, the setup described in figure 4.3 was realized experimentally for measuring different fiber links. Those links consisted of rolls of fiber of known approximate length spliced together so that a fault could be induced between them. For each experiment, the fiber link under test was analyzed with measurements performed with the aid of a commercially available OTDR which were used as reference for comparison and evaluation of the proposed method.

A KAP-10 (1557 nm) laser was used to generate the CW transmission signal with direct modulation. Figure 7.3 shows the continuous wave response of the laser for different driving bias current values. It indicates the linear operating range of the laser between $50 \mathrm{~mA}$ and $90 \mathrm{~mA}$ approximately.

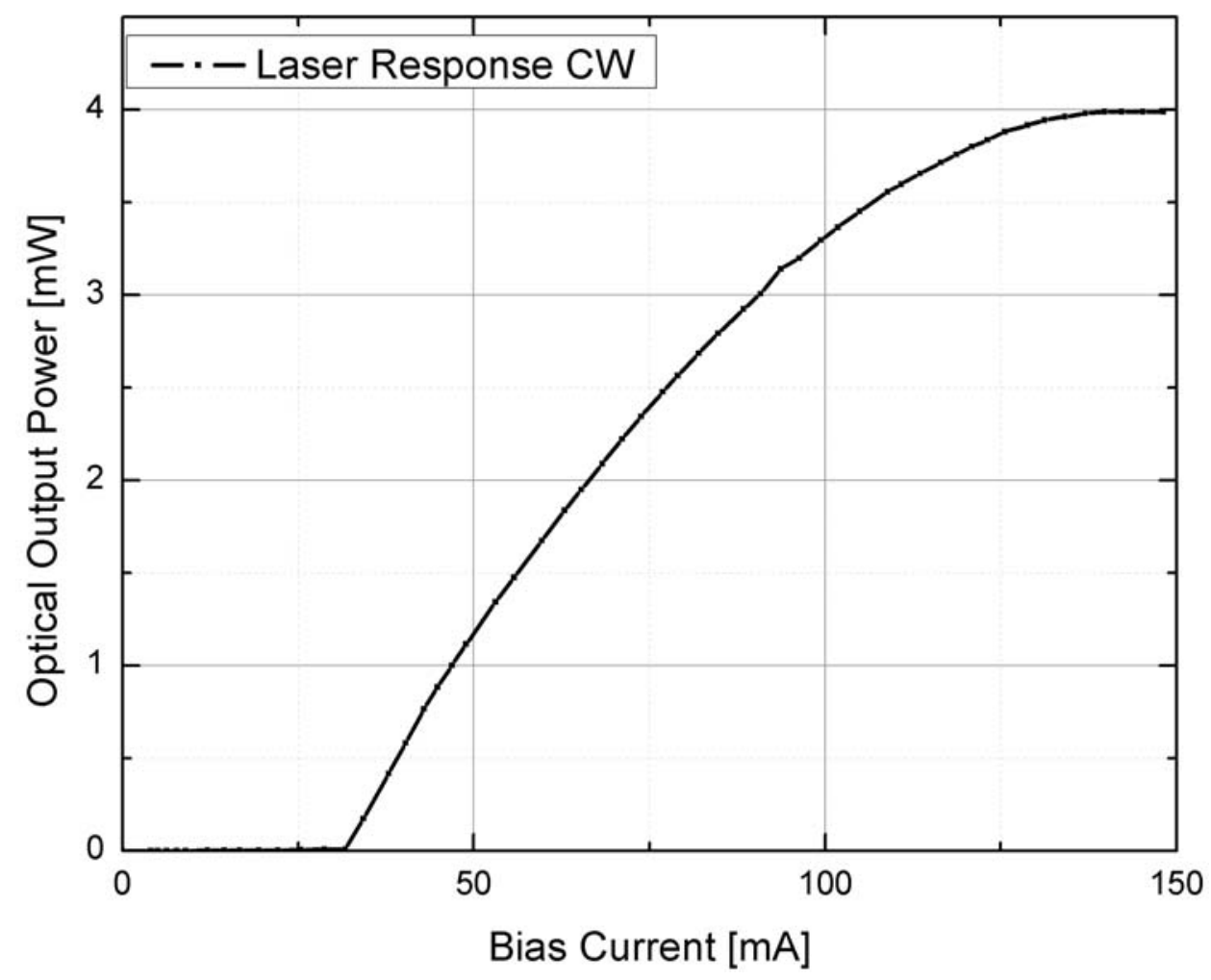

Figure 7.3: KAP-10 laser characterization: optical output power vs. bias current

The modulating signal used consisted only of the sinusoidal tone generated by a Lock-in Amplifier, where the frequency of the sine was swept with the aid of a computer algorithm. Since the frequency range of the Lock-in Amplifier is only about $100 \mathrm{kHz}$, it should not be a problem to overlay additional services in the same baseband electrical sub-carrier, nor shoud it have any effect on the higher frequency sub-carriers of the data channel. Data ac- 
quired from the Lock-in Amplifier was processed in MATLAB ${ }^{\circledR}$, using the data fitting algorithm described before, with the full model for the detected signal represented by equation 5 -10. These initial results are presented and discussed next.

In experiments involving point to point links, a set of two or more fiber rolls where spliced in the lab with one end connected to port 2 of the circulator and a fault was induced close to the splice location. Figure 7.4 represents the setting which emulates the fiber link.

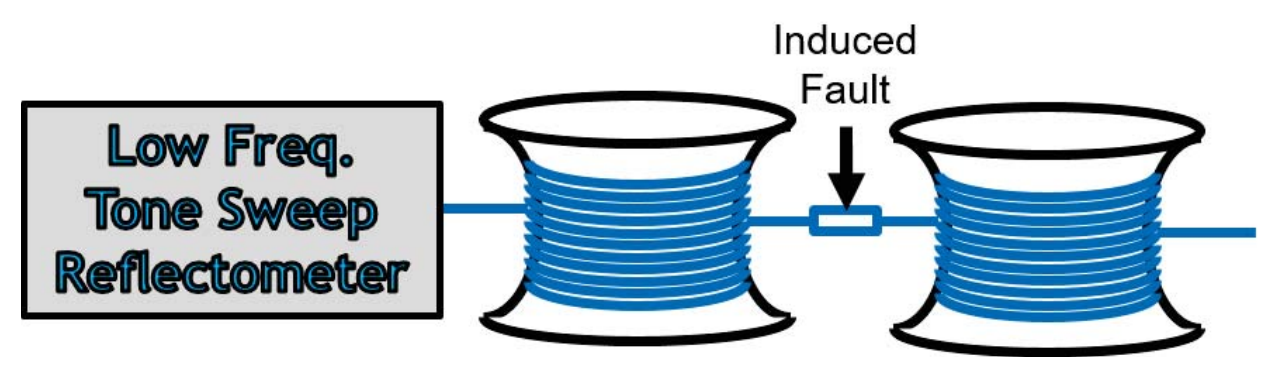

Figure 7.4: Experimental setting for generic point-to-point link

Figure 7.5 presents the output of the proposed method when a long $12 \mathrm{~km}$ fiber was monitored. The graph shows the results for the detected signal when no loss has occurred (reference signal), as well as the cases when a $2 d B$ fault, a $3 d B$ fault and a $4 d B$ fault close to a fiber break (high loss factor) are induced about $8 \mathrm{~km}$ away from the input. As can be seen, the proposed solution achieved good results, matching the experimental detected signal curve and estimating the fault parameters, in accordance with the simulations. Fault location was found with less that $30 \mathrm{~m}$ accuracy, while corresponding intensity was less that $0.6 d B$ away from the reference OTDR measurement.

Another view of these results is given in figure 7.6, where the amplitude and phase of the experimental data and estimated measurements are presented in a polar plot for each of the induced fault cases. As was the case in the examples presented in the simulations section, the frequency dependence would be given by the z-axis perpendicular to the 2-dimensional plot. More experiments with long fiber links can further validate these results.

A similar experiment was realized with a short $1.1 \mathrm{~km}$ fiber link. In this case, the link included 6 spliced fiber sections, one of approximate length $500 \mathrm{~m}$ and five of length close to $100 \mathrm{~m}$. The splicing resulted in the presence of small pre-existing known faults measured with a commercial OTDR and characterized as the fiber signature prior to monitoring. This situation is equivalent to the case of an initial status measurement at installation, for example. A $3 d B$ fault was induced at different splice positions, one at a time. 

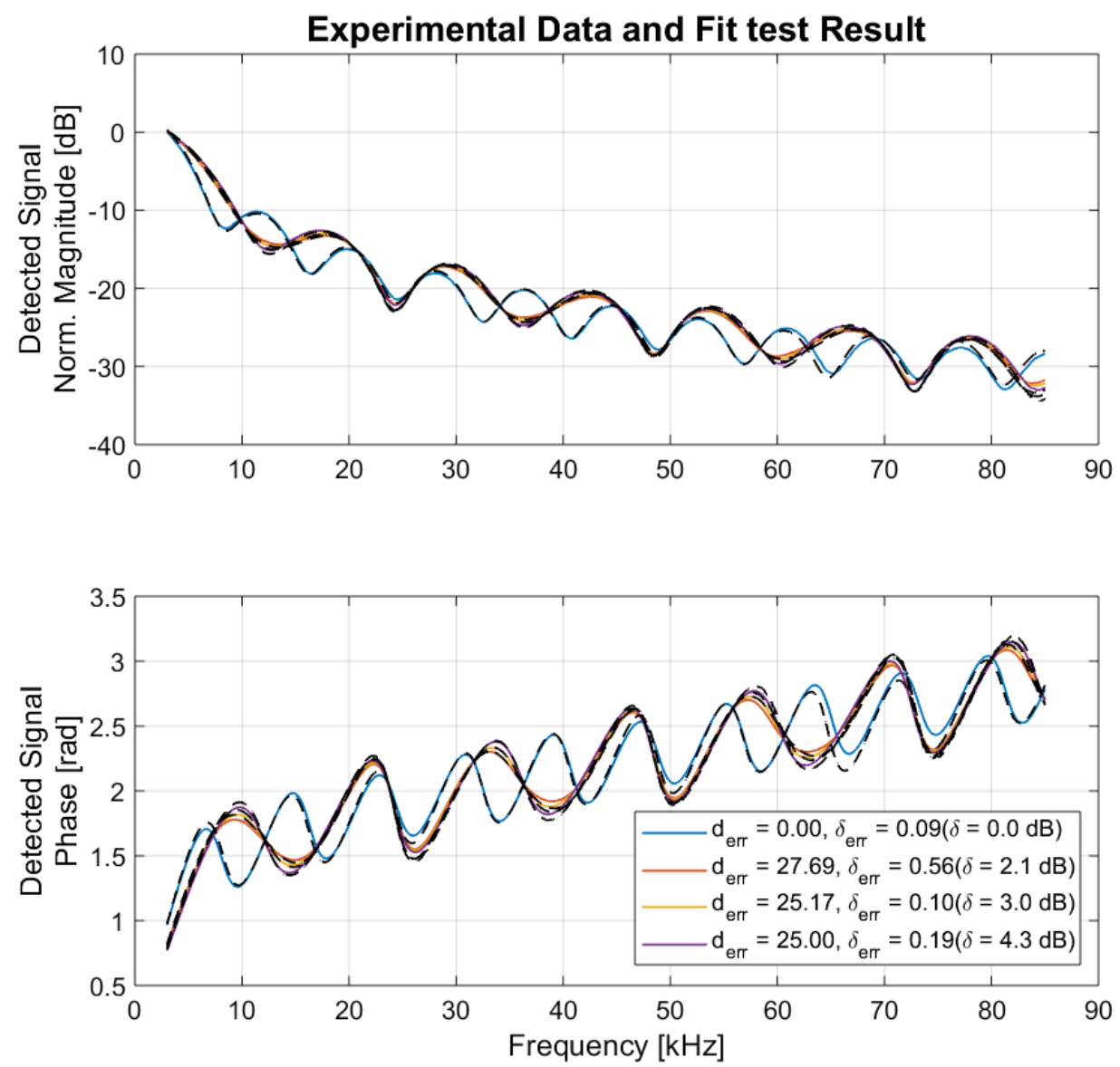

Figure 7.5: Experimental results for $12 \mathrm{~km}$ single fiber link

A polar plot for a selection of results from the monitoring test using the method proposed in this research are presented in figure 7.7. The figure shows a graphic comparison between measured and estimated data corresponding to induced faults close to the splice locations at approximately $300 \mathrm{~m}, 400 \mathrm{~m}, 700 \mathrm{~m}$ and $800 \mathrm{~m}$ from the fiber link input end. In all cases tested the solution estimate is very close to the commercial OTDR measurement, indicating the reliability of the information. Actual measured fault positions and intensity are compared to the estimated values in table 7.1. Is is clear that the method's ability to determine a fault location in a short fiber is very precise, while it fails to determine fault intensity correctly close to the fiber end as predicted. This outcome is in very good agreement with expected results from simulations previously discussed.

This experiment was executed considering an estimation model using the full description for the detected signal presented in equation 5-11 as the goal function for optimization. It was possible to verify the method's accuracy as a fault occurs at different positions along the fiber link. Figure 7.8 shows the location estimation for the different positions evaluated, as well as the corresponding fault intensity estimation error in each case. Estimation error 

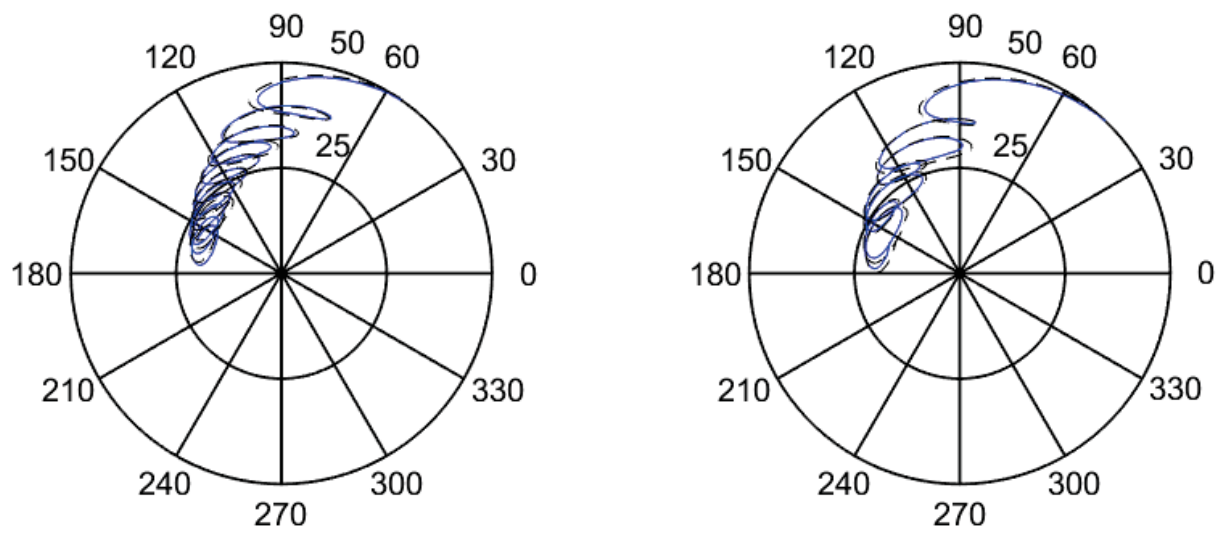

Data: $\mathrm{d}=8438 \mathrm{~m}, \delta=0.00 \mathrm{~dB}$

$---\cdot$ Fit: $\mathrm{d}^{\prime}=8438 \mathrm{~m}, \delta^{\prime}=-0.09 \mathrm{~dB}$
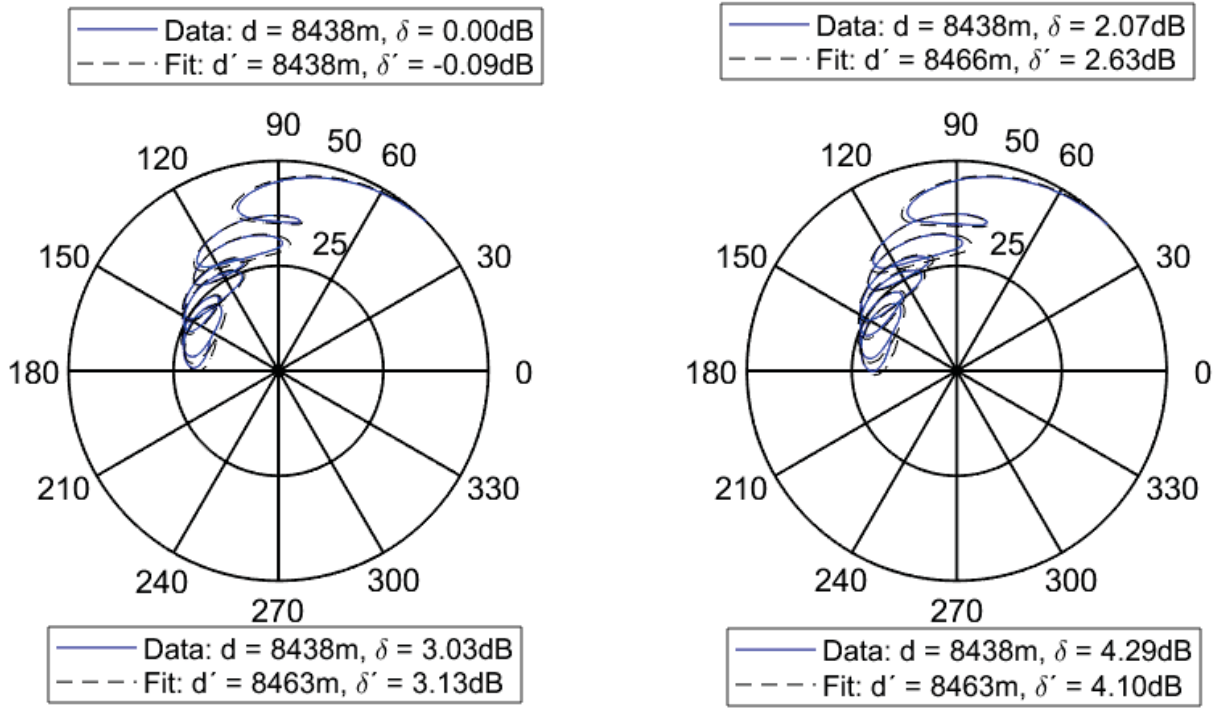

Figure 7.6: Polar plots for experiment with $12 \mathrm{~km}$ single fiber link

Table 7.1: Measured and estimated faults for $1.1 \mathrm{~km}$ experiment

\begin{tabular}{ccccc}
$\begin{array}{c}\text { Link } \\
\text { Length }\end{array}$ & $\begin{array}{c}\text { Reference } \\
\mathbf{d}\end{array}$ & $\begin{array}{c}\text { Reference } \\
\delta\end{array}$ & $\begin{array}{c}\text { Estimated } \\
\left(\mathbf{d}_{\text {est }}\right)\end{array}$ & $\begin{array}{c}\text { Estimated } \\
\left(\delta_{\text {est }}\right)\end{array}$ \\
\hline & $108 m$ & & $124 m$ & $3.13 d B$ \\
& $213 m$ & & $221 m$ & $3.44 d B$ \\
& $318 m$ & & $319 m$ & $3.50 d B$ \\
Short Link & $424 m$ & & $414 m$ & $3.55 d B$ \\
$(1.1 k m)$ & $508 m$ & $\approx 3 d B$ & $508 m$ & $2.43 d B$ \\
& $718 m$ & & $617 m$ & $2.26 d B$ \\
& $824 m$ & & $722 m$ & $2.43 d B$ \\
& $929 m$ & & $831 m$ & $2.45 d B$ \\
& $1034 m$ & & $940 m$ & $1.96 d B$ \\
& & $1027 m$ & $1.45 d B$ \\
\hline
\end{tabular}

represented by the error bars in the graph correspond to the dissonance found for multiple simulation scenarios with each possible fault position discussed in the simulations section. It is clear that accuracy is diminished as the fault is induced closer to the end of the fiber. 

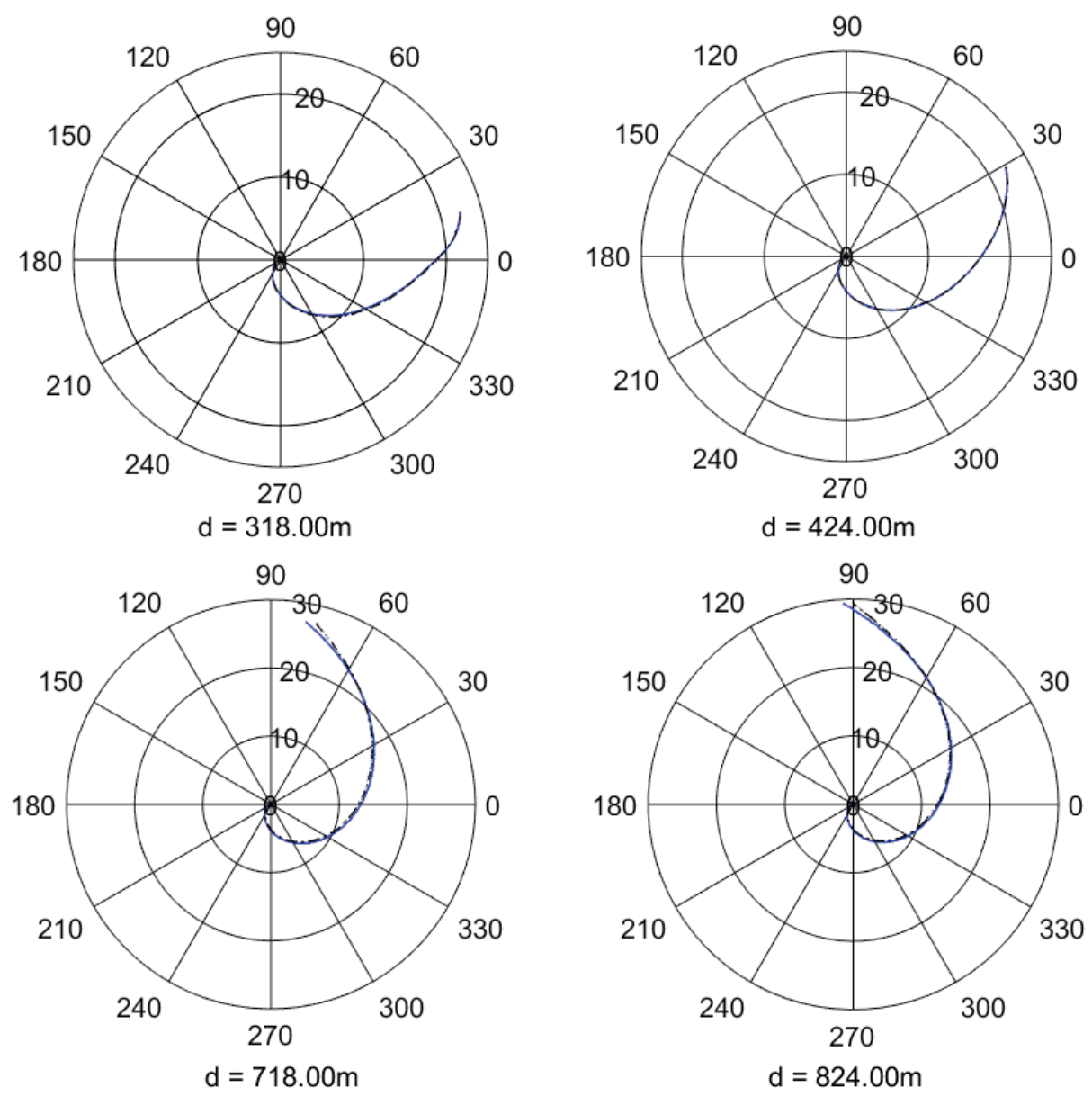

Figure 7.7: Polar plots for experiment with $1.1 \mathrm{~km}$ single fiber link

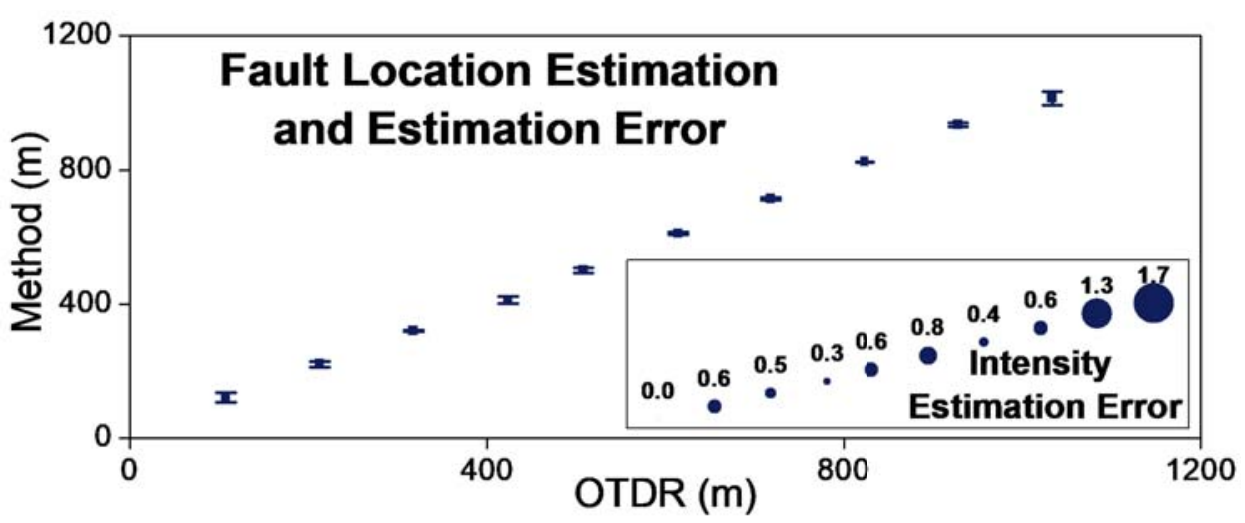

Figure 7.8: Experiment with $1.1 \mathrm{~km}$ single fiber link comparing $3 d B$ induced fault at various positions

The results from these initial experiments show that mensuration of an unknown fault in a point-to-point link can be obtained with good accuracy with the proposed method, even using the full model for the detected backscattered signal. The outcome of the tests obtained provide good enough information for monitoring purposes.

The proposed method's capability to locate and measure very small faults 

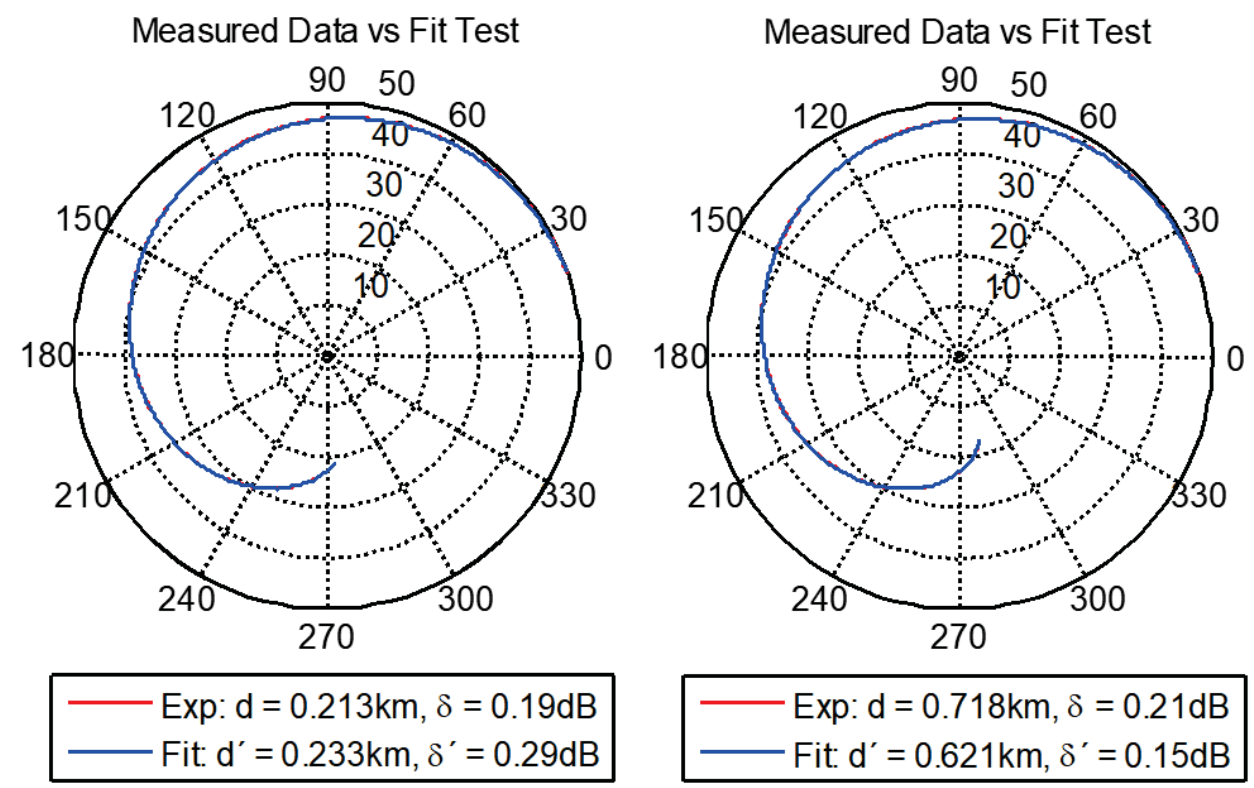

Figure 7.9: Experimental result of estimation of very small faults at about $200 \mathrm{~m}$ and at $700 \mathrm{~m}$ from the input to a $1.1 \mathrm{~km}$ fiber

was verified by submitting the data from the experiment with the $1.1 \mathrm{~km}$ fiber when no loss was induced and omitting one of the measured splice losses from the fiber signature. As an example, figure 7.9 shows the results for locating the splice loss of $0.21 \mathrm{~dB}$ (measured by the OTDR) at $700 \mathrm{~m}$ from the fiber input. This outcome attests to the adaptability and reliability of the monitoring solution proposed. Nonetheless, as discussed in the previous chapter, it was not possible to apply the complete mathematical model described directly for data measurements acquired in a TDM-PON setting, as the algorithm did not converge.

\section{3}

\section{Experiments with Differential Mathematical Model for Estimation}

Although the results above were in very good agreement with fault measurements obtained offline with the aid of a commercially available OTDR, simulation tests indicate the alternative solution using the differential mathematical model might be more accurate and even work well for branched networks. As discussed, using the extension of the complete mathematical model for the signal response for point-to-point links to PONs in optimization may not converge. In order to overcome this limitation and try to improve on pointto-point link results, the differential model was used.

Furthermore, a different laser was chosen which provided a better linear response as described by figure 7.10 - Mitsubishi FU-68 PDF-5. This is important to allow for a larger modulation depth as previously discussed. 
Additionally, during trial experiments, coherent Rayleigh noise effect was noticed as mentioned before. This noise affects mainly the lower frequency range, where the monitoring method proposed here is employed and thus needs to be treated. In the experiments presented next, the setup was modified as described in figure 7.2 .

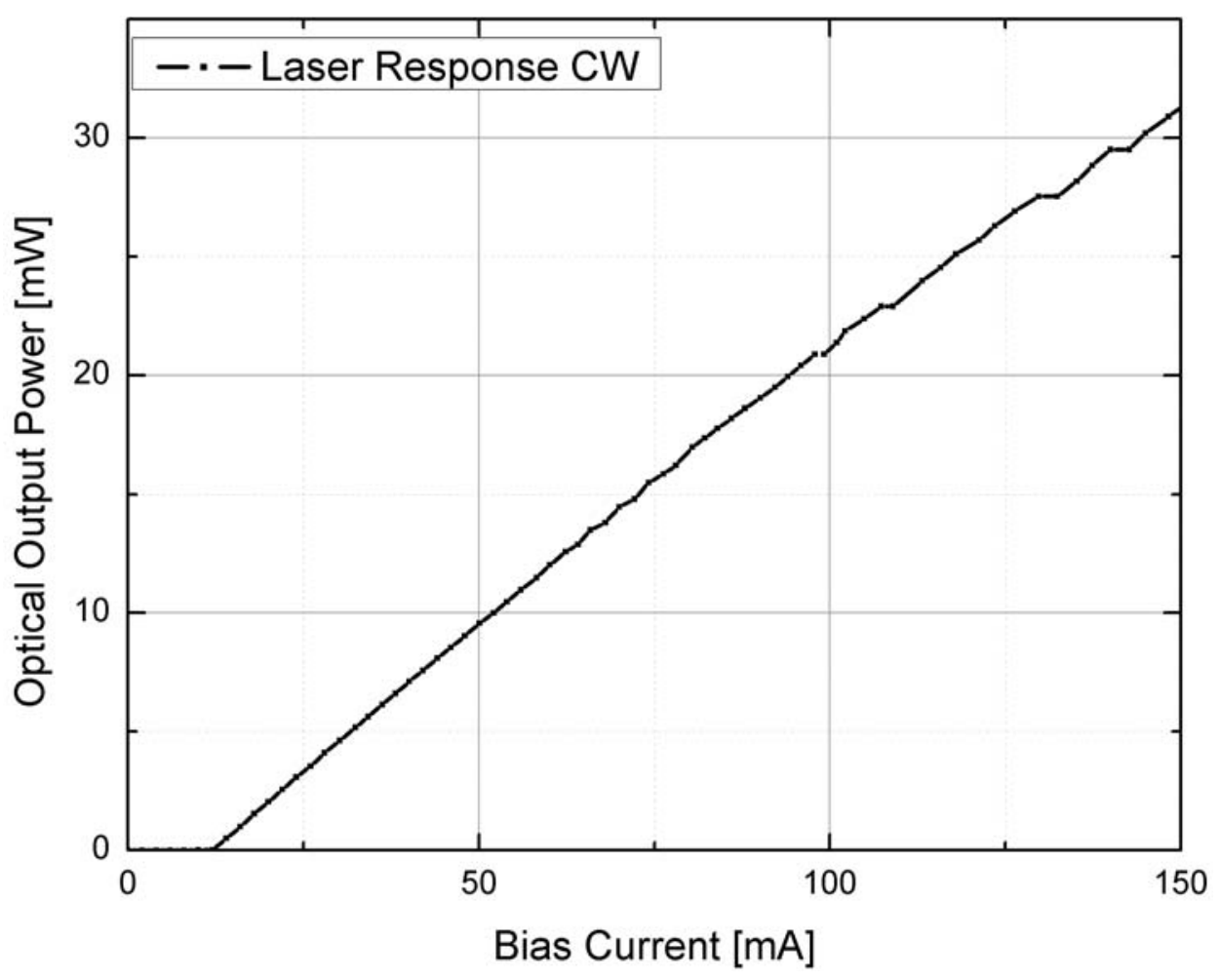

Figure 7.10: Mitsubishi laser characterization: optical output power vs. bias current

The laser bias was set at $70.8 \mathrm{~mA}$, to ensure linearity of the response. A triangular wave with $200 \mathrm{mV}$ peak-to-peak amplitude and $2.096 \mathrm{~Hz}$ frequency was added to the modulation of the laser in order to reduce the CRN effect. The Lock-in amplifier generated a sine wave with $700 \mathrm{~m} V_{\text {rms }}$ corresponding to approximately $1 V$ peak-to-peak amplitude, which provided a good modulation depth. The detector gain was set to $1 \times 10^{3}(30 d B)$.

A $3 \mathrm{~km}$ long single fiber link consisting of two spliced rolls of fiber of approximate lengths $850 \mathrm{~m}$ and $2150 \mathrm{~m}$ was used in the point-to-point experimental trials after the changes presented. Various fault intensities were induced near the splice, between the two fiber rolls and measured with the proposed method and with a commercially available OTDR. Figures 7.11 and 7.12 show the OTDR reference measurements used to evaluate the accuracy of the method.

The output of the optimization estimation for fault location and intensity in each case was evaluated. Figures 7.13 and 7.14 present the results using 


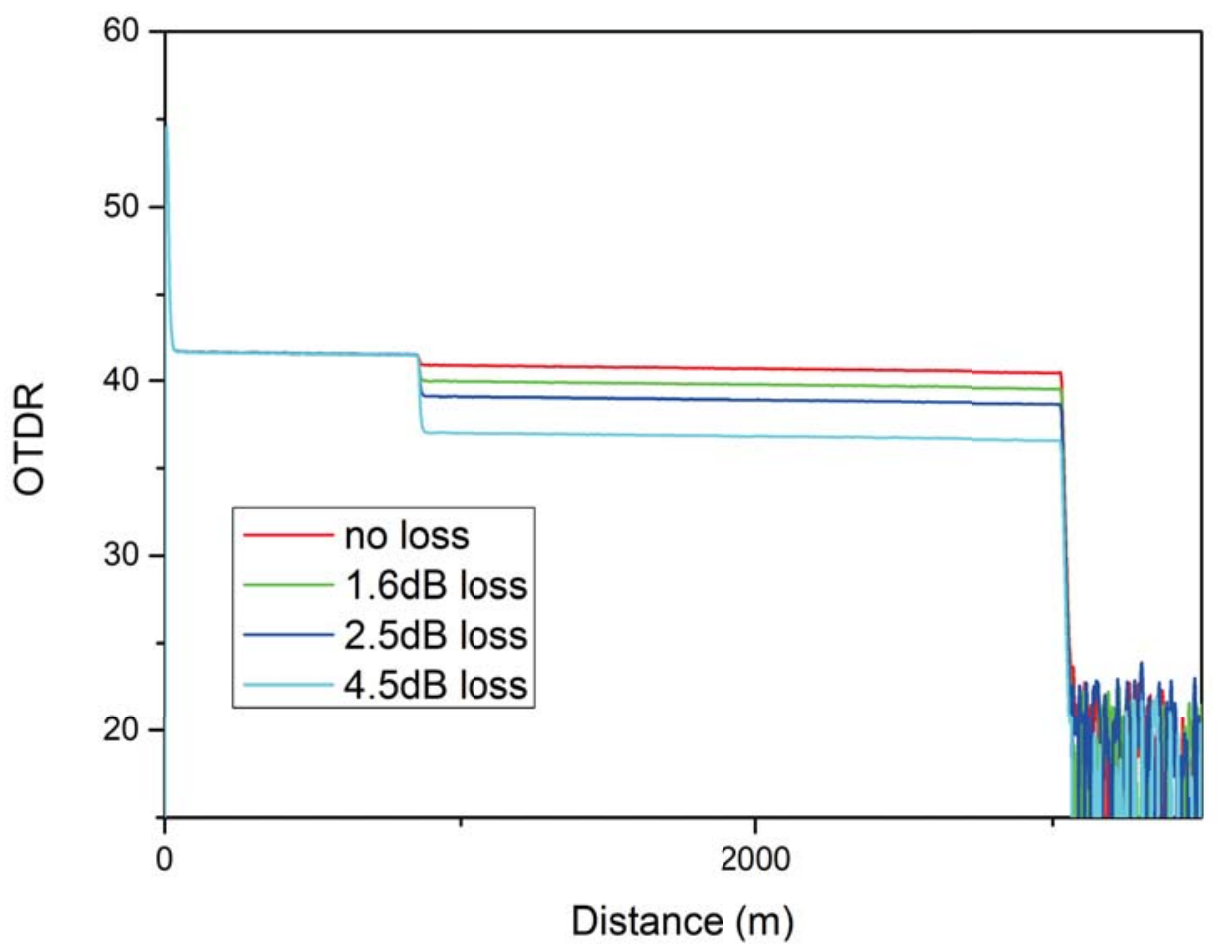

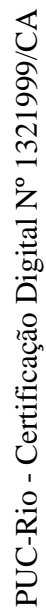

Figure 7.11: OTDR measurements for different induced faults at $850 \mathrm{~m}$ of a $3 \mathrm{~km}$ link

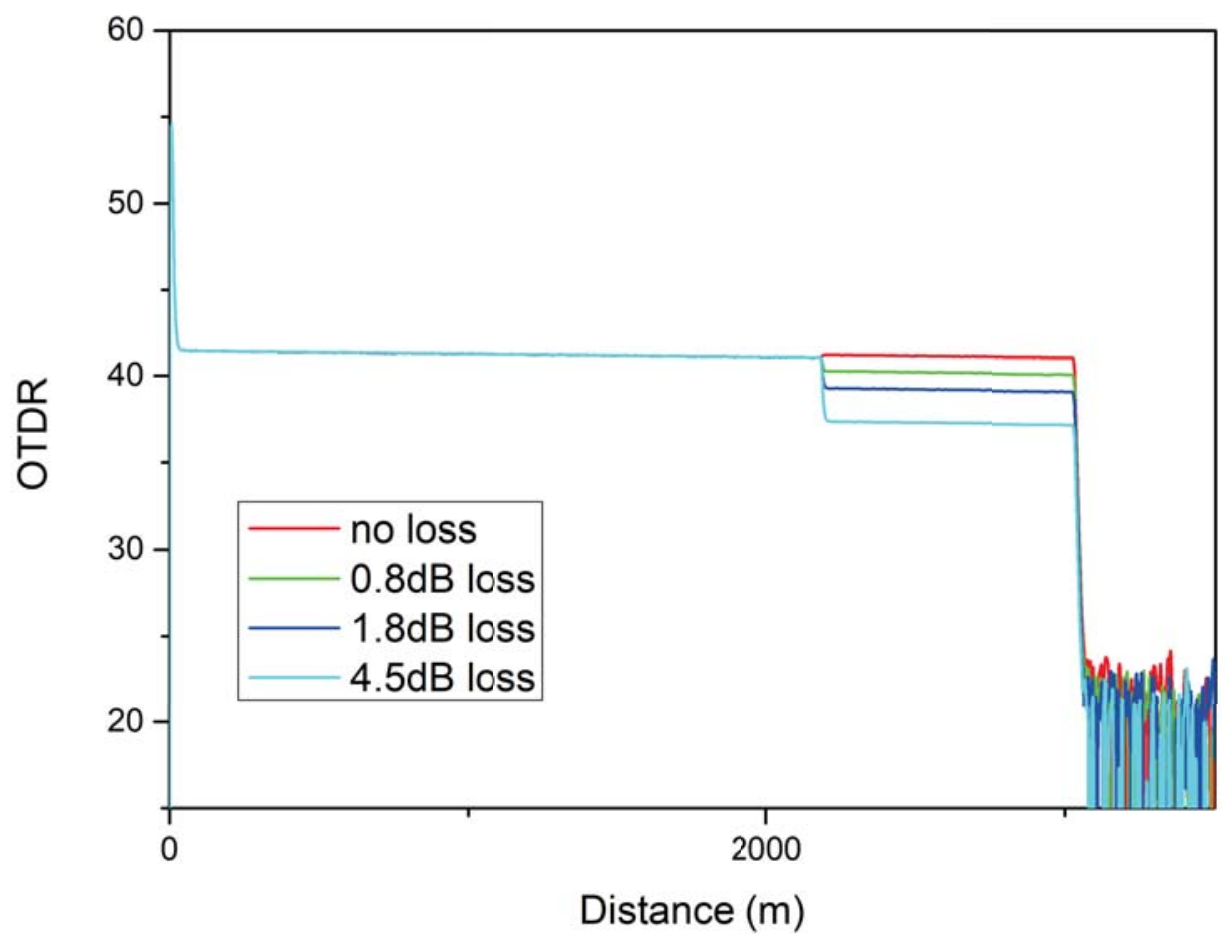

Figure 7.12: OTDR measurements for different induced faults at $2150 \mathrm{~m}$ of a $3 k m$ link

the differential mathematical model for the point-to-point compared to the measured data obtained experimentally for the different fault positions. It is 
clear that the method using the differential mathematical model provides very good results in this case, even better than those achieved with the full model which were presented earlier.
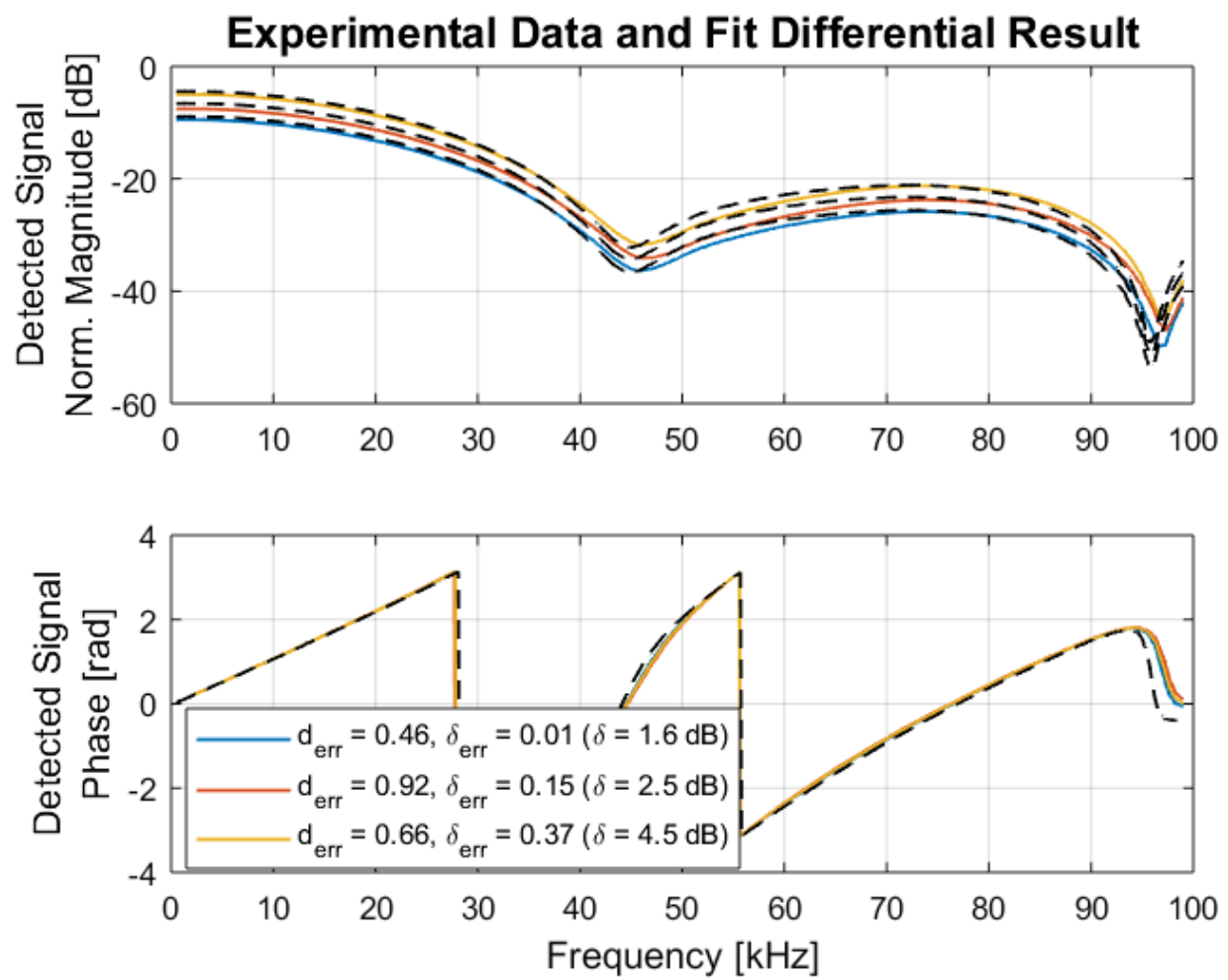

Figure 7.13: Comparison between estimated differential curve and measured data for various faults at $850 \mathrm{~m}$ of a $3 \mathrm{~km}$ link

Next a branched network setting was created by splicing different fiber rolls into the input and the output extremities of a $1: 4$ optical power splitter mimicking a PON. Rolls of fiber were spliced to the input to the passive splitter and to each of its output ports. In select ports two fiber rolls were split in a similar fashion to what was done in point-to-point experiments so that a fault could be induced close to the splice position. Figure 7.15 represents the experimental setting for this kind of link.

The first PON considered (PON 1) included a $520 \mathrm{~m}$ feeder line and four branches corresponding to lengths $L_{1}=1090 \mathrm{~m}, L_{2}=1430 \mathrm{~m}, L_{3}=1555 \mathrm{~m}$ and $L_{4}=1657 \mathrm{~m}$. A fault was induced at position $d=1045 \mathrm{~m}$ in the branch of corresponding length $L_{3}=1555 \mathrm{~m}$ as shown in the OTDR reference measurement presented in figure 7.16. The OTDR shows the apparent loss of $0.8 d B$. The actual fault in the branch is masked by the backscattered light contribution from the branches longer that the fault location and must be calculated using the formula 7-2 suggested in [81]. The fault measured by the OTDR is the apparent loss $\delta_{a p p}$. The actual fault intensity is $\delta$ and $N_{L}$ is the number of branches longer than the fault location. 

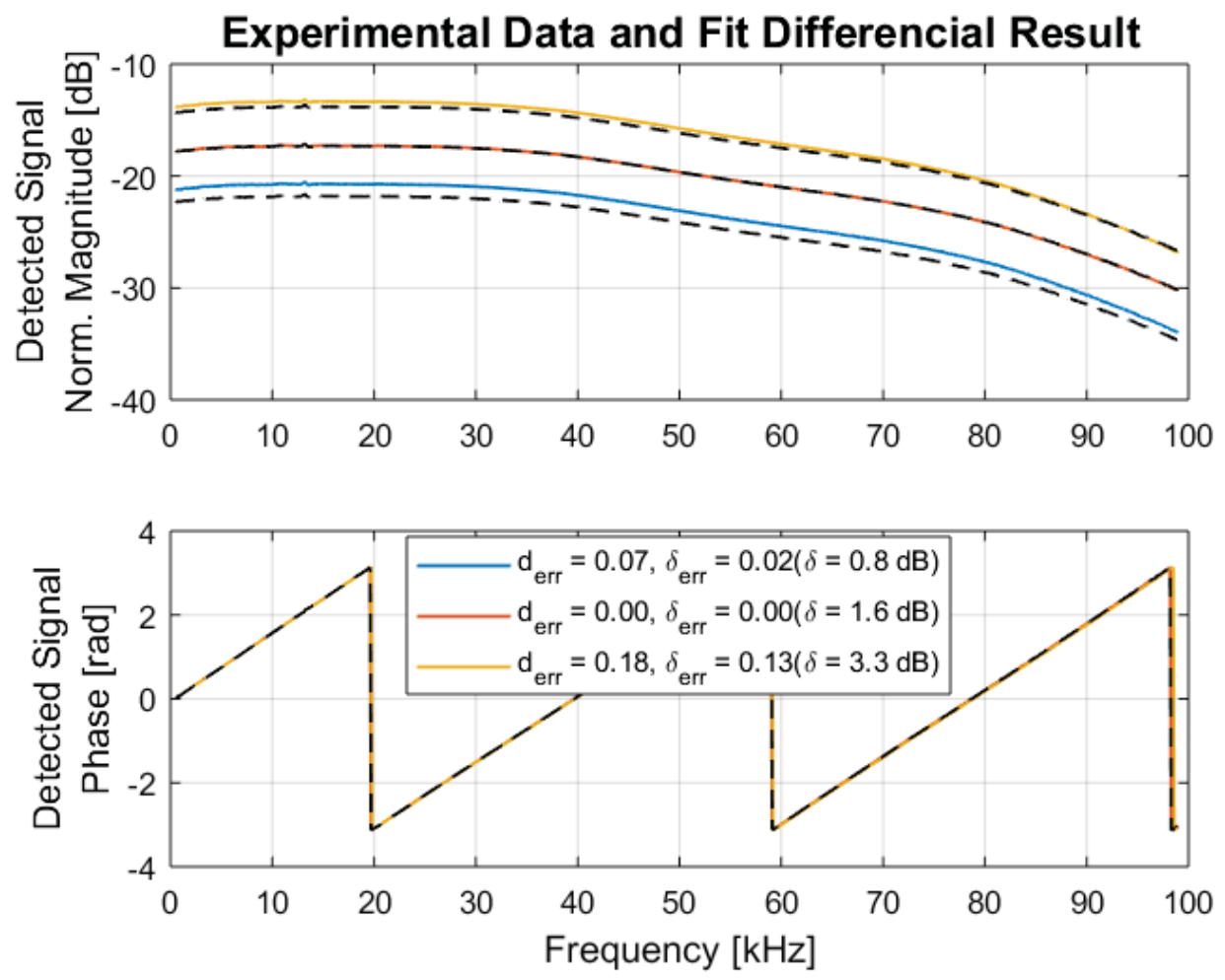

Figure 7.14: Comparison between estimated differential curve and measured data for various faults at $2150 \mathrm{~m}$ of a $3 \mathrm{~km}$ link

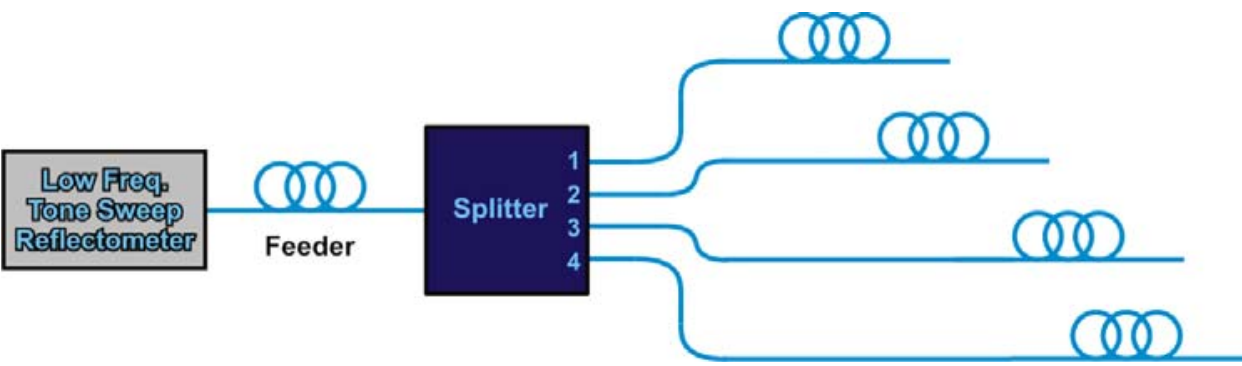

Figure 7.15: Experimental setting for generic PON link

$$
\delta_{a p p}=5 \log \left(\frac{N_{L}-1+10^{0.2 \delta}}{N_{L}}\right)
$$

The equation may be reversed to obtain the actual loss as shown in 7-3. This way, the fault measured in PON 1 is actually approximately $2.3 d B$.

$$
\delta=5 \log \left[N_{L}\left(10^{0.2 \delta_{a p p}}-1\right)+1\right]
$$

Subsequently, another branched network setting was implemented in a similar fashion with a 1 : 4 optical power splitter mimicking a second PON. This network (PON 2) included a $1044 \mathrm{~m}$ feeder line and four branches corresponding to lengths $L_{1}=1612 \mathrm{~m}, L_{2}=2076 \mathrm{~m}, L_{3}=2180 \mathrm{~m}$ and $L_{4}=2464 \mathrm{~m}$. This time, a fault was induced first in the branch of corresponding length $L_{2}=2076 \mathrm{~m}$ at position $d=1560 \mathrm{~m}$ with apparent loss shown in the 


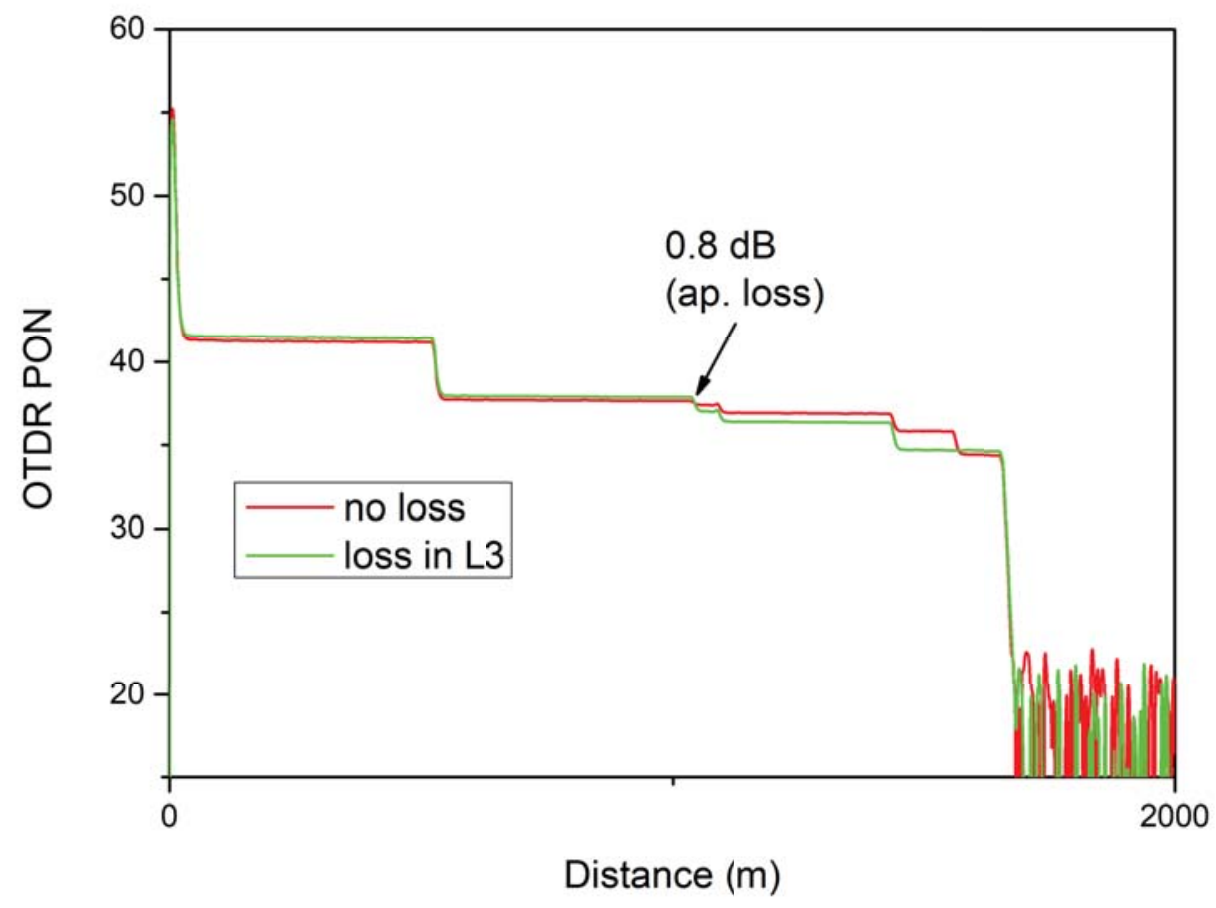

Figure 7.16: OTDR measurements for PON setting (1).

OTDR reference measurement of $0.5 d B$. In a separate experiment, a fault was induced in the branch of corresponding length $L_{4}=2464 \mathrm{~m}$ at position $d=1959 m$ with apparent loss shown in the OTDR reference measurement of $1 d B$, while the first fault had been removed. Figure 7.17 shows the reference measurements obtained in these experiments. The actual fault in each case i again calculated using the formula 7-3.

The differential model for the PON was used as the goal function for the optimization. The signal generated from the estimation outcome for fault location, including branch information, and fault intensity is compared to the parameters measured as shown in figure 7.18. Numerical values obtained for each experimental setting are presented in table 7.2, where the accuracy of the output can be verified. The experiments show very good estimate for locating of a fault position and corresponding branch, within less than $20 \mathrm{~m}$ with a tolerable error of the fault intensity estimation, below $0.8 d B$.

Experimental results are in good agreement with the simulated outcome in all settings tested, thus validating the methodology and mathematical models employed. Furthermore, the latter results validate the insight that use of the differential mathematical model yields more accurate results than the complete description of the detected signal.

It is important to note that experimental tests were performed with laser driver bias current close to $70 \mathrm{~m} A$, while the modulation signal excursion was around $40 \mathrm{~mA}$. Initial experiments with the KAP-10 laser used the full 


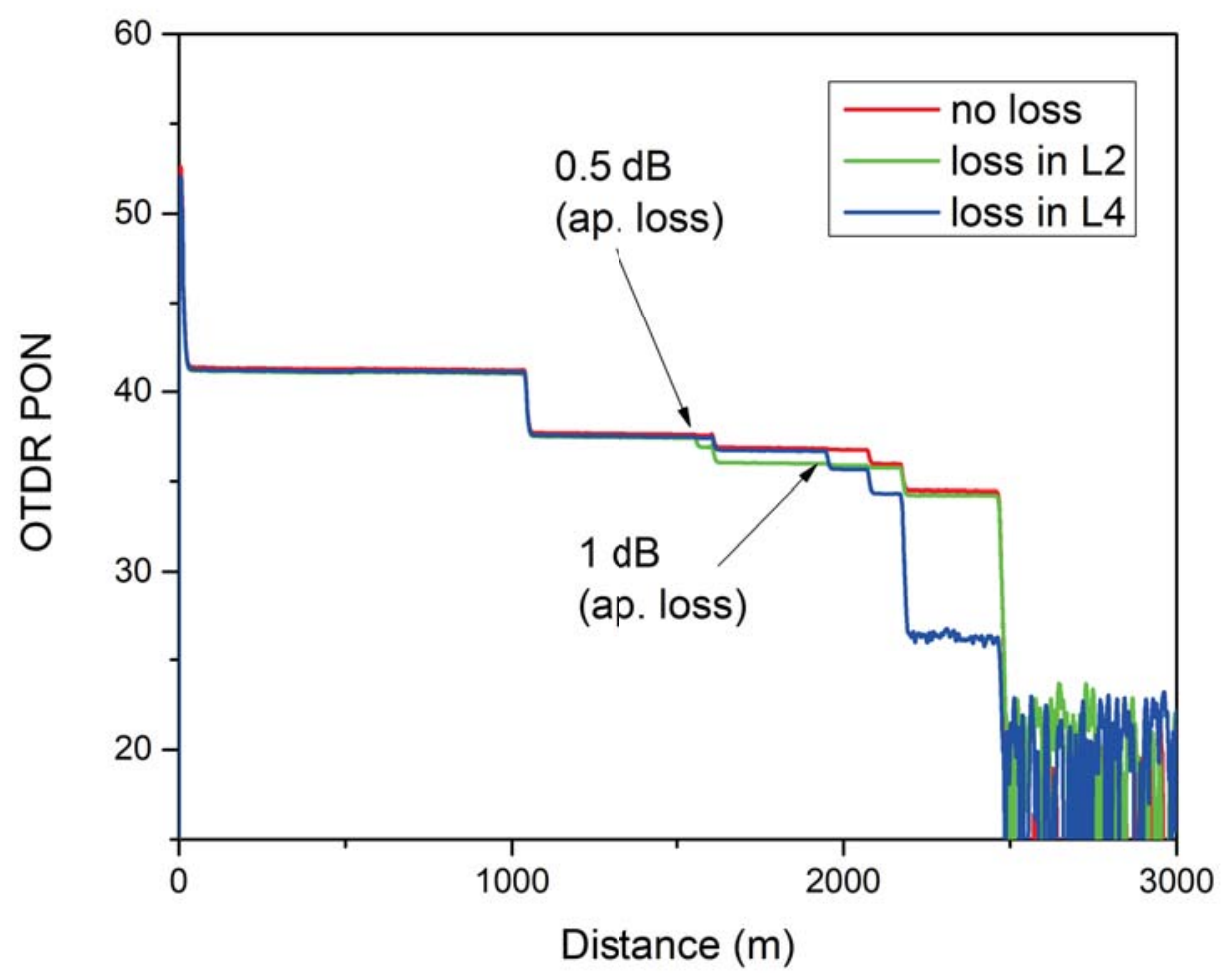

Figure 7.17: OTDR measurements for PON setting (2).

excursion of the linear region of the laser and although in the latter experiments with the Mitsubishi laser a fraction of the linear region was left unexplored. In practice, the modulating signal excursion including all of the SCM channels is limited to the linear region of the laser source, so that less current amplitude is available for monitoring in either case. This means the monitoring signal power in a real application will be diminished, nonetheless, simulations showed that, even under lower SNR, the methods suggested perform sufficiently well. 

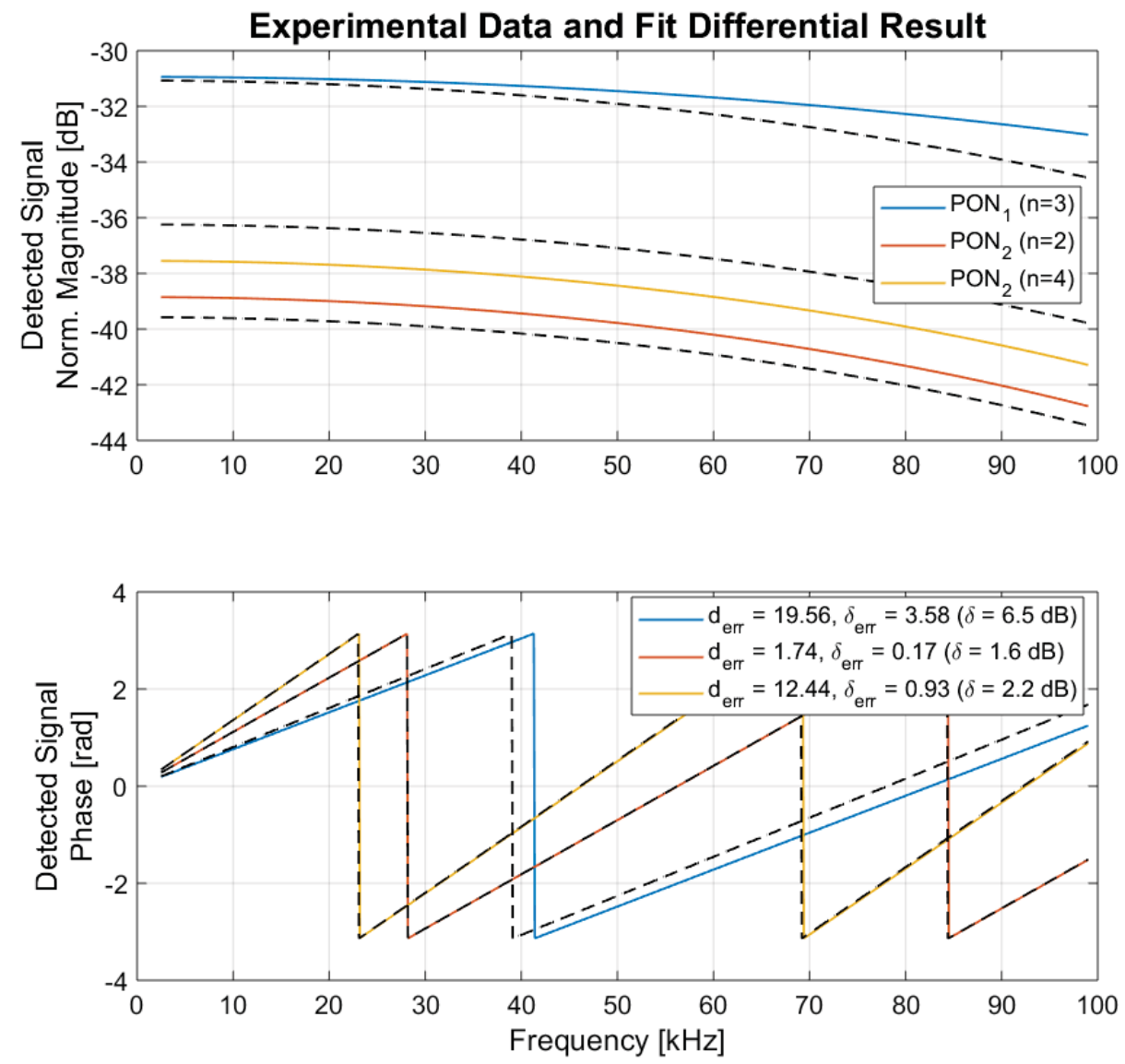

Figure 7.18: Comparison between estimared differential curve and measured data for PON experiments. 
Table 7.2: Differential Estimation Result for PON mimicking setting

\begin{tabular}{|c|c|c|c|c|}
\hline Experiment & $\begin{array}{c}\text { Measurement } \\
\text { Type }\end{array}$ & $d$ & $\delta$ & Branch \\
\hline \multirow{3}{*}{ PON 1} & $\begin{array}{c}\text { OTDR+ } \\
\text { Equation } 7-3\end{array}$ & $1046 m$ & $2.3 d B$ & $L_{3}$ \\
\hline & Method & $1066 \mathrm{~m}$ & $3.1 d B$ & $L_{3}$ \\
\hline & Accuracy & $20 m$ & $0.8 d B$ & $\checkmark$ \\
\hline \multirow{6}{*}{ PON 2} & $\begin{array}{c}\text { OTDR+ } \\
\text { Equation } 7-3\end{array}$ & $1560 m$ & $1.6 d B$ & $L_{2}$ \\
\hline & Method & $1562 m$ & $1.4 d B$ & $L_{2}$ \\
\hline & Accuracy & $2 m$ & $-0.2 d B$ & $\checkmark$ \\
\hline & $\begin{array}{c}\text { OTDR+ } \\
\text { Equation 7-3 }\end{array}$ & $1959 m$ & $2.2 d B$ & $L_{4}$ \\
\hline & Method & $1971 m$ & $3.1 d B$ & $L_{4}$ \\
\hline & Accuracy & $12 m$ & $0.7 d B$ & $\checkmark$ \\
\hline
\end{tabular}




\section{8 \\ Conclusions}

In this work a novel technique which can be used to monitor fiber links in either point-to-point links or branched passive networks was proposed. The solution takes into consideration the use of sub-carrier multiplexing recommended for $5 \mathrm{G}$ communication networks by suggesting that the lowest frequency range of the base band sub-carrier be used for monitoring the fiber access link alongside data transmission.

The proposed technique is a suitable solution for embedded monitoring of passive optical networks, focusing on those employing analog radio over fiber (a-RoF) that are likely to employ sub-carrier modulation (SCM-PON). It provides a promising cost effective approach to locating and measuring both punctual and distributed faults from the central office through constant monitoring, while employing mostly the same equipment used for data transmission.

A model describing the behavior of the Rayleigh backscattered signal detected at the input to the fiber when a sine with frequency swept within a few $k H z$ is used to modulate the transmitting laser was presented and discussed for both point-to-point and PON link types. Simulation shows that the estimated fault results can reach the theoretical data accurately and experimental results match reference measurements closely.

An alternative solution was presented considering a measurement of the fiber link evaluated prior to the fault occurrence was available. The mathematical models for either point-to-point links or branched networks were simplified to differential models which were compared to the difference between the stored measurement and new data. Simulations using this technique showed even better accuracy than the former method.

It has been shown in this research that the monitoring technique proposed provides a suitable measurement for either point-to-point links or TDM-PON with reasonable accuracy. Simulated values show the method can achieve estimation error of less than $5 \mathrm{~m}$ and less than $0.01 \mathrm{~dB}$ for position and intensity of an unknown faults respectively in most cases. Experimental results lead to less than $1 \mathrm{~m}$ estimation error for the position and less than $0.5 \mathrm{~dB}$ estimation error for intensity of an unknown faults in point to point links. PON experiments present accuracy within $20 \mathrm{~m}$ for estimated position and less than 
$1 d B$ for estimated fault intensity in comparison to real loss calculated from apparent loss. Furthermore the actual faulty branch of a PON was determined correctly every time for both simulated and experimental settings.

Some limitations of the model were discussed and ways to overcome them were suggested. Although limited experimental settings were evaluated, they show good agreement with the theoretical and simulated results and, thus provide satisfactory proof-of-concept outcome. Furthermore the experimental results validate the insight in comparing the two mathematical methods employed.

The frequency swept tone signal described in the setup can be added to the base band sub-carrier signal in SCM-RoF transmission along with other signaling and synchronization information. Since the monitoring signal range with the suggested solution is up to a few tens of $k H z$, it shouldn't interfere with these other services. This way constant monitoring which is unlikely to disrupt data services is possible. The only necessary additional equipment are an optical circulator, a low frequency detector and low frequency electrical circuitry and digital signal processing capable of generating the monitoring signal and analyzing the detected data.

An extension of the model to include the analysis of reflective elements is clearly straightforward. Each punctual reflective element presents as a tone in the detected signal with fixed phase proportional to the position of the reflective element. Therefore, these faults can be treated separately and their estimation is left aside for future work. Furthermore, studies on the cross talk between the monitoring and data signals are assumed to be negligible since the separation between sub-carriers is necessary to support this kind of modulation and the subject is also left outside the scope of this work. 


\section{Bibliography}

[1] HUI, R.; O'SULLIVAN, M. Fiber optic measurement techniques. Academic Press, 2009.

[2] SENIOR, J. M.; JAMRO, M. Y. Optical fiber communications: principles and practice. Pearson Education, 2009. Cap. 14.

[3] DERICKSON, D. Fiber optic test and measurement. Hewlett-Packard professional books. Prentice Hall PTR, 1998.

[4] RAD, M. M.; FOULI, K.; FATHALLAH, H. A.; RUSCH, L. A.; MAIER, M. Passive optical network monitoring: challenges and requirements. Communications Magazine, IEEE, v. 49, n. 2, p. s45-S52, 2011.

[5] International Telecommunication Union, Geneva. Supplement 55 to itu$t$ g-series recommendations: radio-over-fibre (rof) technologies and their applications, 2015.

[6] BRINKMEYER, E. Backscattering in single-mode fibres. Electronics Letters, v. 16, n. 9, p. 329-330, 1980.

[7] GYSEL, P.; STAUBLI, R. K. Statistical properties of Rayleigh backscattering in single-mode fibers. Lightwave Technology, Journal of, v. 8, n. 4, p. 561567, 1990.

[8] FROGGATT, M. E.; GIFFORD, D. K. Rayleigh backscattering signatures of optical fibers - Their properties and applications. In: Optical Fiber Communication Conference. c2013. p. OW1K-6.

[9] BARNOSKI, M. K.; ROURKE, M. D.; JENSEN, S. M.; MELVILLE, R. T. Optical Time Domain Reflectometer. Applied Optics, New York, v. 16, n. 9, p. 2375-2379, 1977.

[10] MACDONALD, R. Frequency domain optical reflectometer. Applied Optics, New York, v. 20, n. 10, p. 1840-1844, 1981.

[11] SCHMUCK, H.; HEHMANN, J.; STRAUB, M.; PFEIFFER, T. Embedded OTDR techniques for cost-efficient fibre monitoring in optical access networks. 
2006 European Conference on Optical Communications Proceedings, ECOC 2006, p. 10-11, 2006.

[12] CHEN, W.; De Mulder, B.; VANDEWEGE, J.; QIU, X. Z.; BAUWELINCK, J.; BAEKELANDT, B. A novel technique for low-cost embedded nonintrusive fiber monitoring of P2MP optical access networks. OFC/NFOEC 2007 - Optical Fiber Communication and the National Fiber Optic Engineers Conference 2007, , n. January, p. 4-6, 2007.

[13] KUZNIA, C.; AHADIAN, J.; POMMER, D.; HAGAN, R.; BACHTA, P.; WONG, M.; KUSUMOTO, K.; SKENDZIC, S.; TABBERT, C.; BERANEK, M. W. Novel high-resolution OTDR technology for multi-Gbps transceivers. Conference on Optical Fiber Communication, Technical Digest Series, p. 2-4, 2014.

[14] DARCIE, T. E. Subcarrier Multiplexing for Multiple-Access Lightwave Networks. Journal of Lightwave Technology, v. 5, n. 8, p. 1103-1110, 1987.

[15] DARCIE, T. E. Subcarrier Multiplexing for Lightwave Networks and Video Distribution Systems. IEEE Journal on Selected Areas in Communications, v. 8, n. 7, p. $1240-1248,1990$.

[16] ARSAT, M.; IDRUS, S. M.; NAWAWI, N. M. Performance analysis of sub carrier multiplexed system for radio over fiber technology. Proceedings of IEEE 2008 6th National Conference on Telecommunication Technologies and IEEE 2008 2nd Malaysia Conference on Photonics, NCTT-MCP 2008, , n. August, p. 226-229, 2008.

[17] NAKAZAWA, M. Rayleigh backscattering theory for single-mode optical fibers. JOSA, v. 73, n. 9, p. 1175-1180, 1983.

[18] GYSEL, P.; STAUBLI, R. K. Spectral properties of Rayleigh backscattered light from single-mode fibers caused by a modulated probe signal. Lightwave Technology, Journal of, v. 8, n. 12, p. 1792-1798, 1990.

[19] STAUBLI, R. K.; GYSEL, P. Statistical properties of single-mode fiber Rayleigh backscattered intensity and resulting detector current. Communications, IEEE Transactions on, v. 40, n. 6, p. 1091-1097, 1992.

[20] BARNOSKI, M.; JENSEN, S. Fiber waveguides: a novel technique for investigating attenuation characteristics. Applied Optics, New York, v. 15, n. 9, p. 2112-2115, 1976. 
[21] ANDERSON, D. R.; JOHNSON, L. M.; BELL, F. G. Troubleshooting optical fiber networks: Understanding and using optical time-domain reflectometers. Academic Press, 2004.

[22] BARNOSKI, M. K.; PERSONICK, S. Measurements in fiber optics. Proceedings of the IEEE, v. 66, n. 4, p. 429-441, 1978.

[23] HEALEY, P. Review of long wavelength single-mode optical fiber reflectometry techniques. Journal of lightwave technology, v. 3, n. 4, p. 876-886, 1985.

[24] HEALEY, P. Fading in heterodyne OTDR. Electronics letters, v. 20, n. 1, p. 30-32, 1984.

[25] HEALEY, P. Complementary code sets for OTDR. Electronics Letters, v. 25, n. 11, p. $692-693,1989$.

[26] IZUMITA, H.; FURUKAWA, S.-I.; KOYAMADA, Y.; SANKAWA, I. Fading noise reduction in coherent OTDR. Photonics Technology Letters, IEEE, v. 4, n. 2, p. 201-203, 1992.

[27] NAZARATHY, M.; NEWTON, S. A.; GIFFARD, R.; MOBERLY, D.; SISCHKA, F.; TRUTNA JR, W.; FOSTER, S. Real-time long range complementary correlation optical time domain reflectometer. Lightwave Technology, Journal of, v. 7, n. 1, p. 24-38, 1989.

[28] NAZARATHY, M.; NEWTON, S.; TRUTNA, W. Complementary correlation OTDR with three codewords. Electronics Letters, v. 1, n. 26, p. 70-71, 1990.

[29] SUMIDA, M. OTDR performance enhancement using a quaternary FSK modulated probe and coherent detection. IEEE photonics technology letters, v. 7, n. 3, p. 336-338, 1995.

[30] MENDIETA, F.; TREVINO, A.; MARTINEZ, C. Complementary sequence correlations with applications to refflectometry studies. Instrumentation and Development, v. 3, n. 6, p. 37-46, 1996.

[31] PARK, N.; LEE, J.; PARK, J.; SHIM, J. G.; YOON, H.; KIM, J. H.; KIM, K.; BYUN, J.-O.; BOLOGNINI, G.; LEE, D. et al. Coded optical time domain reflectometry: principle and applications. In: Asia-Pacific Optical Communications. c2007. p. 678129-678129.

[32] HEALEY, P. Optical time domain reflectometry: a performance comparison of the analogue and photon counting techniques. Optical and quantum electronics, v. 16, n. 3, p. 267-276, 1984. 
[33] ERAERDS, P.; LEGRÉ, M.; ZHANG, J.; ZBINDEN, H.; GISIN, N. Photon counting OTDR: advantages and limitations. Journal of Lightwave Technology, v. 28, n. 6, p. 952-964, 2010.

[34] IIDA, H.; KOSHIKIYA, Y.; ITO, F.; TANAKA, K. High-sensitivity coherent optical time domain reflectometry employing frequency-division multiplexing. Journal of Lightwave Technology, v. 30, n. 8, p. 1121-1126, 2012.

[35] YUKSEL, K.; WUILPART, M.; MOEYAERT, V.; MÉGRET, P. Optical frequency domain reflectometry: a review. In: ICTON: 2009 11th International Conference on Transparent Optical Networks, Vols. c2009. v. 1. p. 723-727.

[36] EICKHOFF, W.; ULRICH, R. Optical frequency domain reflectometry in single-mode fiber. Applied Physics Letters, New York, v. 39, n. 9, p. 693695, 1981.

[37] SHADARAM, M.; KURIGER, W. L. Using the optical frequency domain technique for the analysis of discrete and distributed reflections in an optical fiber. Applied optics, v. 23, n. 7, p. 1092-1095, 1984.

[38] GHAFOORI-SHIRAZ, H.; OKOSHI, T. Optical-fiber diagnosis using opticalfrequency-domain reflectometry. Optics letters, v. 10, n. 3, p. 160-162, 1985.

[39] NAKAYAMA, J.; IIZUKA, K.; NIELSEN, J. Optical fiber fault locator by the step frequency method. Applied optics, v. 26, n. 3, p. 440-443, 1987.

[40] NEWTON, S. et al. Novel approaches to optical reflectometry. In: Instrumentation and Measurement Technology Conference, 1990. IMTC-90. Conference Record., 7th IEEE. c1990. p. 329-333.

[41] SORIN, W. V.; DONALD, D.; NEWTON, S. A.; NAZARATHY, M. Coherent FMCW reflectometry using a temperature tuned Nd: YAG ring laser. Photonics Technology Letters, IEEE, v. 2, n. 12, p. 902-904, 1990.

[42] GLOMBITZA, U.; BRINKMEYER, E. Coherent frequency-domain reflectometry for characterization of single-mode integrated-optical waveguides. Lightwave Technology, Journal of, v. 11, n. 8, p. 1377-1384, 1993.

[43] VON DER WEID, J.; PASSY, R.; MUSSI, G.; GISIN, N. On the characterization of optical fiber network components with optical frequency domain reflectometry. Journal of Lightwave Technology, v. 15, n. 7, p. 1131-1141, 1997. 
[44] FAN, X.; KOSHIKIYA, Y.; ITO, F. Phase-noise-compensated optical frequency-domain reflectometry. Quantum Electronics, IEEE Journal of, v. 45, n. 6, p. 594-602, 2009.

[45] FAN, X.; KOSHIKIYA, Y.; ITO, F. 2-cm spatial resolution over $40 \mathrm{~km}$ realized by bandwidth-division phase-noise-compensated OFDR. In: Optical Fiber Communication Conference. c2011. p. OMF3.

[46] ITO, F.; FAN, X.; KOSHIKIYA, Y. Long-range coherent OFDR with light source phase noise compensation. Journal of Lightwave Technology, v. 30, n. 8, p. 1015-1024, 2012.

[47] SOLLER, B.; WOLFE, M.; FROGGATT, M. Polarization resolved measurement of Rayleigh backscatter in fiber-optic components. OFC Technical Digest, Los Angeles, 2005.

[48] HANGAI, S.; TAKI, Y. Detection of faults in short fiber by the phase compensated reflectometer. Instrumentation and Measurement, IEEE Transactions on, v. 39, n. 1, p. 238-241, 1990.

[49] CHEN, X.; ZHANG, X.; YAN, B.; LI, J.; CHEN, X.; TU, G.; LIANG, Y.; NI, Z.; CULSHAW, B.; DONG, F. Research on coherent Rayleigh backscattering and signal processing method in phase-OTDR. In: OFS2012 22nd International Conference on Optical Fiber Sensor. c2012. p. 8421A3-8421A3.

[50] QIN, Z.; ZHU, T.; CHEN, L.; BAO, X. High sensitivity distributed vibration sensor based on polarization-maintaining configurations of phase-OTDR. Photonics Technology Letters, IEEE, v. 23, n. 15, p. 1091-1093, 2011.

[51] GU, X.; CHU, C.; SABLATASH, M. Optical wavelet domain reflectometry. In: Time-Frequency and Time-Scale Analysis, 1994., Proceedings of the IEEE-SP International Symposium on. c1994. p. 158-161.

[52] CABALLERO, D. V.; ALMEIDA, R.; URBAN, P.; COSTA, J.; VON DER WEID, J.; CHEN, J. SCM/WDM-PON with in-service baseband embedded OTDR monitoring. Optics Communications, Amsterdam, v. 356, p. 250-255, 2015.

[53] DER WEID, V. et al. Experimental demonstration of SCM-PON monitoring with baseband embedded OTDR. In: . c2016. p. 1-4.

[54] VILLAFANI CABALLERO, D. R.; YNOQUIO HERRERA, L. E.; CASTRO DO AMARAL, G.; URBAN, P. J.; VON DER WEID, J. P. Experimental Demonstration of SCM-PON Monitoring with Baseband Embedded OTDR. Fiber and Integrated Optics, v. 36, n. 1-2, p. 59-67, 2017. 
[55] CABALLERO, D. V.; HERRERA, L.; VON DER WEID, J.; URBAN, P. LowCost Embedded OTDR Monitoring for Direct Modulation Analog Radio over Fiber. In: .

[56] HERRERA, L. E. Y.; F. CALLIARI1, D. V. C.; AMARAL, G. C.; URBAN, P. J.; VON DER WEID, J. P. Transmitter-Embedded AMCC, LTE-A and OTDR signal for Direct Modulation Analog Radio over Fiber Systems.

[57] SCHMUCK, H.; HEHMANN, J.; STRAUB, M.; PFEIFFER, T. Embedded OTDR techniques for cost-efficient fibre monitoring in optical access networks. In: 2006 European Conference on Optical Communications. c2006.

[58] CHEN, W.; DE MULDER, B.; VANDEWEGE, J.; QIU, X.; BAUWELINCK, J.; BAEKELANDT, B. A Novel Technique for Low-Cost Embedded Nonintrusive Fiber Monitoring of P2MP Optical Access Networks. 2007.

[59] YUKSEL, K.; MOEYAERT, V.; WUILPART, M.; MÉGRET, P. Optical layer monitoring in passive optical networks (PONs): a review. In: Proceedings of 10th Anniversary International Conference on Transparent Optical Networks (ICTON 2008), Athens, Greece. c2008. p. 92-98.

[60] SANKAWA, I.; FURUKAWA, S.-I.; KOYAMADA, Y.; IZUMITA, H. Fault location technique for in-service branched optical fiber networks. Photonics Technology Letters, IEEE, v. 2, n. 10, p. 766-768, 1990.

[61] TANAKA, K.; TATEDA, M.; INOUE, Y. Measuring the individual attenuation distribution of passive branched optical networks. Photonics Technology Letters, IEEE, v. 8, n. 7, p. 915-917, 1996.

[62] CHAN, C. K.; TONG, F.; CHEN, L.; SONG, J.; LAM, D. A passive surveillance scheme for passive branched optical networks. In: Optical Fiber Communication. OFC 97., Conference on. c1997. p. 51-52.

[63] CAVIGLIA, F.; DI BIASE, V. C.; GNAZZO, A. Optical maintenance in PONs. Optical Fiber Technology, v. 5, n. 4, p. 349-362, 1999.

[64] URBAN, P. J.; VALL-LLOSERA, G.; MEDEIROS, E.; DAHLFORT, S. Fiber plant manager: an OTDR-and OTM-based PON monitoring system. Communications Magazine, IEEE, v. 51, n. 2, p. S9-S15, 2013.

[65] URBAN, P.; GETANEH, A.; VON DER WEID, J.; TEMPORÃO, G. P.; VALLLLOSERA, G.; CHEN, J. Detection of fiber faults in passive optical networks. Journal of Optical Communications and Networking, v. 5, n. 11, p. 11111121, 2013. 
[66] XIA, L.; HUANG, D.; XU, J.; LIU, D. Simultaneous and precise fault locating in WDM-PON by the generation of optical wideband chaos. Optics letters, v. 38, n. 19, p. 3762-3764, 2013.

[67] FEIGEL, B.; VAN ERPS, J.; KHODER, M.; BERI, S.; JEURIS, K.; VAN GOIDSENHOVEN, D.; WATTÉ, J.; THIENPONT, H. Optical TimeDomain Reflectometry Simulations of Passive Optical Networks: A Linear Time-Invariant System Approach for Arbitrary Pulses. Journal of Lightwave Technology, v. 32, n. 17, p. 3008-3019, 2014.

[68] CAVIGLIA, F.; BIASE, V. D. Optical maintenance in PONs. 24th European Conference on Optical Communication. ECOC '98 (IEEE Cat. No.98TH8398), v. 1, n. September, p. 349-362, 1998.

[69] TOMITA, N.; TAKASUGI, H.; ATOBE, N.; NAKAMURA, I.; TAKAESU, F.; TAKASHIMA, S. Design and performance of a novel automatic fiber line testing system with OTDR for optical subscriber loops. Lightwave Technology, Journal of, v. 12, n. 5, p. 717-726, 1994.

[70] RAMANITRA, H.; CHANCLOU, P.; ETRILLARD, J.; ANMA, Y.; NAKADA, $\mathrm{H}$.; ONO, $\mathrm{H}$. Optical access network using a self-latching variable splitter remotely powered through an optical fiber link. Optical Engineering, v. 46, n. 4, p. 045007-045007, 2007.

[71] TANAKA, K.; TATEDA, M.; MEMBER, S.; INOUE, Y. Measuring the Individual Attenuation Distribution of Passive Branched Optical Networks. IEEE Photonics Technology Letters, v. 8, n. 7, p. 915-917, 1996.

[72] URBAN, P. J.; MEDEIROS, E.; VALL-LLOSERA, G. WDM-PON fiber-fault automatic detection and Localization with $1 \mathrm{~dB}$ event sensitivity in drop links. In: National Fiber Optic Engineers Conference. c2012. p. NM2K-4.

[73] YUKSEL, K.; DUPONT, S.; ROBETTE, L.; HAMOIR, D.; FROIDURE, J. OTDR-based fault surveillance method for tree-structured passive optical networks. In: Int. Symp. on Services and Local Access, ISSLS. c2004.

[74] YEH, C.-H.; CHI, S. Optical fiber-fault surveillance for passive optical networks in S-band operation window. Optics express, v. 13, n. 14, p. 54945498, 2005.

[75] TAKUSHIMA, Y.; CHUNG, Y. Optical reflectometry based on correlation detection and its application to the in-service monitoring of WDM passive optical network. Optics express, v. 15, n. 9, p. 5318-5326, 2007. 
[76] LÁZARO VILLA, J. A.; POLO QUEROL, V.; COSTA, L.; TEIXEIRA, A. Viability of in-service, low-cost and spatially unambiguous OTDR monitoring in TDM-and WDM-PON access networks. In: 11th International Conference on Transparent Optical Networks, 2009. c2009. p. 53-57.

[77] RAD, M. M.; FATHALLAH, H. A.; RUSCH, L. A. Fiber fault PON monitoring using optical coding: Effects of customer geographic distribution. IEEE Transactions on Communications, v. 58, n. 4, p. 1172-1181, 2010.

[78] NAIM, N. F.; AB-RAHMAN, M. S.; BAKARMAN, H. A.; BAKAR, A. A. A. Real-time monitoring in passive optical networks using a superluminescent LED with uniform and phase-shifted fiber Bragg gratings. Journal of Optical Communications and Networking, v. 5, n. 12, p. 1425-1430, 2013.

[79] HASEGAWA, T.; INOUE, A. Monitoring of drop optical fibers in 32branched PON using $1.65 \mu \mathrm{m}$ pulse-OCDR. In: National Fiber Optic Engineers Conference. c2009. p. NWA5.

[80] ESMAIL, M. A.; FATHALLAH, H. Novel coding for PON fault identification. Communications Letters, IEEE, v. 15, n. 6, p. 677-679, 2011.

[81] TEMPORAO, G. P.; DE FARIA, G. V.; VON DER WEID, J. P.; URBAN, P. J. Feasibility of centralized PON monitoring using PON-tuned OTDR. In: Ultra Modern Telecommunications and Control Systems and Workshops (ICUMT), 2012 4th International Congress on. c2012. p. 545-551.

[82] AMARAL, G. C.; BALDIVIESO, A.; GARCIA, J. D.; VILLAFANI, D. C.; LEIBEL, R. G.; HERRERA, L. E.; URBAN, P. J.; VON DER WEID, J. P. A low-frequency tone sweep method for in-service fault location in subcarrier multiplexed optical fiber networks. Journal of Lightwave Technology, v. 35, n. 10, 2017.

[83] RENATA G. LeIBEL, P. J. U.; VON DER WEID, J. P. Fault Localization and Estimation Method using Sub-Carrier Multiplexed Low Frequency Tone Sweep. In: Oasis 6 Conference and Exhibition on Optics and Electro-Optics.

[84] VON DER, W. J. P.; DO, C.; GUSTAVO, A.; GOLDMAN LEIBEL, R.; VILLAFANI CABALLERO, D. R. MONITORING OPTICAL FIBRE LINK, Nov. 30 2017. US Patent 20,170,346,550.

[85] AGRAWAL, G. Fiber-optic communication systems. Wiley Series in Microwave and Optical Engineering. Wiley, 2012. 
[86] WOOD, T. H.; LINKE, R. A.; KASPER, B. L.; CARR, E. C. Observation of coherent Rayleigh noise in single-source bidirectional optical fiber systems. Journal of lightwave technology, v. 6, n. 2, p. 346-352, 1988.

[87] STAUBLI, R. K.; GYSEL, P. Crosstalk penalities due to coherent Rayleigh noise in bidirectional optical communication systems. Journal of lightwave technology, v. 9, n. 3, p. 375-380, 1991. 


\section{A}

\section{Differential Signal Model}

In this appendix we expand the backscattered response mathematical model to evaluate the characteristics of the response measured after the occurrence of a single fault which can be exploited in order to detect the fault's position and intensity.

Backscattered response caused by a swept signal in a single fiber link of length $L$ :

$$
s(t)=\sum_{i} R_{i} D P_{0} e^{j\left(2 K d_{i}-\omega t\right)}+\int_{0}^{L} D C P_{0} e^{j(2 K z-\omega t)} \mathrm{d} z
$$

Disregarding the reflective effects, equation A-1 is simplified to be:

$$
s_{0}(t)=\int_{0}^{L} D C P_{0} e^{j(2 K z-\omega t)} \mathrm{d} z
$$

Considering a fault at position $d \in(0, L)$, with power loss intensity $\delta$, and defining $A_{0}=D C P_{0}$, the simplified backscattered response in A-2 becomes:

$$
\begin{aligned}
s(t) & =\int_{0}^{d} A_{0} e^{j(2 K z-\omega t)} \mathrm{d} z+\delta^{2} \int_{d}^{L} A_{0} e^{j(2 K z-\omega t)} \mathrm{d} z \\
& =\int_{0}^{d} A_{0} e^{j(2 K z-\omega t)} \mathrm{d} z+\delta^{2}\left[\int_{0}^{L} A_{0} e^{j(2 K z-\omega t)} \mathrm{d} z-\int_{0}^{d} A_{0} e^{j(2 K z-\omega t)} \mathrm{d} z\right] \\
& =\left(1-\delta^{2}\right) \int_{0}^{d} A_{0} e^{j(2 K z-\omega t)} \mathrm{d} z+\delta^{2} \int_{0}^{L} A_{0} e^{j(2 K z-\omega t)} \mathrm{d} z
\end{aligned}
$$

Solving the integrals in A-2 and A-3, the response has the form of phasors with a sinc-like behavior of their amplitudes:

$$
\begin{aligned}
s_{0}(t) & =A_{0} \frac{\sin (K L)}{K} e^{j K L} e^{-j \omega t} \\
& =S_{0}(f) e^{-j \omega t} \\
s(t) & =\left[\left(1-\delta^{2}\right) A_{0} \frac{\sin (K d)}{K} e^{j K d}+\delta^{2} A_{0} \frac{\sin (K L)}{K} e^{j K L}\right] e^{-j \omega t} \\
& =S(f) e^{-j \omega t}
\end{aligned}
$$

Note that equation A-5 is related to equation A-4 as: 


$$
S(f)=\left(1-\delta^{2}\right) A_{0} \frac{\sin (K d)}{K} e^{j K d}+\delta^{2} S_{0}(f)
$$

Now, let there be an existing known fault at position $d_{0}$, with power loss intensity $\delta_{0}$. The reference signal measured is described by:

$$
s_{0}(t)=\left(1-\delta_{0}^{2}\right) \int_{0}^{d_{0}} A_{0} e^{j(2 K z-\omega t)} \mathrm{d} z+\delta_{0}^{2} \int_{0}^{L} A_{0} e^{j(2 K z-\omega t)} \mathrm{d} z
$$

If a new fault with intensity $\delta$ occurs before the existing fault $d<d_{0}$, the faulty measured signal is described below and has the same properties as A-6.

$$
\begin{aligned}
s(t)= & \left(1-\delta^{2}\right) \int_{0}^{d} A_{0} e^{j(2 K z-\omega t)} \mathrm{d} z+\delta^{2}\left(1-\delta_{0}^{2}\right) \int_{0}^{d_{0}} A_{0} e^{j(2 K z-\omega t)} \mathrm{d} z \\
& +\delta^{2} \delta_{0}^{2} \int_{0}^{L} A_{0} e^{j(2 K z-\omega t)} \mathrm{d} z \\
= & \left(1-\delta^{2}\right) \int_{0}^{d} A_{0} e^{j(2 K z-\omega t)} \mathrm{d} z+\delta^{2} s_{0}(t)
\end{aligned}
$$

For the case where $d>d_{0}$, the solution is slightly more complicated:

$$
\begin{aligned}
s(t)= & \left(1-\delta_{0}^{2}\right) \int_{0}^{d_{0}} A_{0} e^{j(2 K z-\omega t)} \mathrm{d} z+\delta_{0}^{2}\left(1-\delta^{2}\right) \int_{0}^{d} A_{0} e^{j(2 K z-\omega t)} \mathrm{d} z \\
& +\delta_{0}^{2} \delta^{2} \int_{0}^{L} A_{0} e^{j(2 K z-\omega t)} \mathrm{d} z \\
= & \delta_{0}^{2}\left(1-\delta^{2}\right) \int_{0}^{d} A_{0} e^{j(2 K z-\omega t)} \mathrm{d} z+s_{0}(t) \\
& -\delta_{0}^{2}\left(1-\delta^{2}\right) \int_{0}^{L} A_{0} e^{j(2 K z-\omega t)} \mathrm{d} z
\end{aligned}
$$

If the reference signal measured is related to a link with two existing faults in positions $d_{1}$ and $d_{2}$, where $d_{1}<d_{2}$ with corresponding fault intensities $\delta_{1}$ and $\delta_{2}$, it may be expressed as:

$$
\begin{aligned}
s_{0}(t)= & \left(1-\delta_{1}^{2}\right) \int_{0}^{d_{1}} A_{0} e^{j(2 K z-\omega t)} \mathrm{d} z+\delta_{1}^{2}\left(1-\delta_{2}^{2}\right) \int_{0}^{d_{2}} A_{0} e^{j(2 K z-\omega t)} \mathrm{d} z \\
& +\delta_{1}^{2} \delta_{2}^{2} \int_{0}^{L} A_{0} e^{j(2 K z-\omega t)} \mathrm{d} z
\end{aligned}
$$

In this scenario, if a new fault occurs in the beginning of the link $d<d 1$, the measured signal will have the same form as in A-6 as shown next. 


$$
\begin{aligned}
s(t)= & \left(1-\delta^{2}\right) \int_{0}^{d} A_{0} e^{j(2 K z-\omega t)} \mathrm{d} z+\delta^{2}\left(1-\delta_{1}^{2}\right) \int_{0}^{d_{1}} A_{0} e^{j(2 K z-\omega t)} \mathrm{d} z \\
& +\delta^{2} \delta_{1}^{2}\left(1-\delta_{2}^{2}\right) \int_{0}^{d_{2}} A_{0} e^{j(2 K z-\omega t)} \mathrm{d} z+\delta^{2} \delta_{1}^{2} \delta_{2}^{2} \int_{0}^{L} A_{0} e^{j(2 K z-\omega t)} \mathrm{d} z \\
= & \left(1-\delta^{2}\right) \int_{0}^{d} A_{0} e^{j(2 K z-\omega t)} \mathrm{d} z+\delta^{2} s_{0}(t)
\end{aligned}
$$

On the other hand, if $d>d_{2}$, the measured signal has a more complicated form:

$$
\begin{aligned}
s(t)= & \left(1-\delta_{1}^{2}\right) \int_{0}^{d_{1}} A_{0} e^{j(2 K z-\omega t)} \mathrm{d} z+\delta_{1}^{2}\left(1-\delta_{2}^{2}\right) \int_{0}^{d_{2}} A_{0} e^{j(2 K z-\omega t)} \mathrm{d} z \\
& +\delta_{1}^{2} \delta_{2}^{2}\left(1-\delta^{2}\right) \int_{0}^{d} A_{0} e^{j(2 K z-\omega t)} \mathrm{d} z+\delta_{1}^{2} \delta_{2}^{2} \delta^{2} \int_{0}^{L} A_{0} e^{j(2 K z-\omega t)} \mathrm{d} z \\
= & \delta_{1}^{2} \delta_{2}^{2}\left(1-\delta^{2}\right) \int_{0}^{d} A_{0} e^{j(2 K z-\omega t)} \mathrm{d} z+s 0(t) \\
& -\delta_{1}^{2} \delta_{2}^{2}\left(1-\delta^{2}\right) \int_{0}^{L} A_{0} e^{j(2 K z-\omega t)} \mathrm{d} z
\end{aligned}
$$

Finally, when the new fault is somewhere between the existing faults $d_{1}<d<d_{2}$ the signal is described by:

$$
\begin{aligned}
s(t)= & \left(1-\delta_{1}^{2}\right) \int_{0}^{d_{1}} A_{0} e^{j(2 K z-\omega t)} \mathrm{d} z+\delta_{1}^{2}\left(1-\delta^{2}\right) \int_{0}^{d} A_{0} e^{j(2 K z-\omega t)} \mathrm{d} z \\
& +\delta^{2} \delta_{1}^{2}\left(1-\delta_{2}^{2}\right) \int_{0}^{d_{2}} A_{0} e^{j(2 K z-\omega t)} \mathrm{d} z+\delta_{1}^{2} \delta_{2}^{2} \delta^{2} \int_{0}^{L} A_{0} e^{j(2 K z-\omega t)} \mathrm{d} z \\
= & \left(1-\delta^{2}\right) \delta_{1}^{2} \int_{0}^{d} A_{0} e^{j(2 K z-\omega t)} \mathrm{d} z+\delta^{2} s_{0}(t) \\
& +\left(1-\delta^{2}\right)\left(1-\delta_{1}^{2}\right) \int_{0}^{d_{1}} A_{0} e^{j(2 K z-\omega t)} \mathrm{d} z
\end{aligned}
$$

Let us consider now the case of a TDM-PON setup as the monitored link. In this case the device under test (DUT) may be considered as the superposition of $N$ single fiber links, where $N$ is the ratio of the PON splitter. Additionally, the presence of the splitter imposes a fault of intensity $\delta_{s} / N$ at $L_{0}$, corresponding to the end of the feeder fiber, common to all the superposed links.

Disregarding again reflective effects, the backscattered reference signal is described by the following equation: 


$$
\begin{aligned}
s_{0}(t) & =\int_{0}^{L_{0}} A_{0} e^{j(2 K z-\omega t)} \mathrm{d} z+\frac{\delta_{s}^{2}}{N^{2}} \sum_{n=1}^{N} \int_{L_{0}}^{L_{n}} A_{0} e^{j(2 K z-\omega t)} \mathrm{d} z \\
& =\left(1-\frac{\delta_{s}^{2}}{N}\right) \int_{0}^{L_{0}} A_{0} e^{j(2 K z-\omega t)} \mathrm{d} z+\frac{\delta_{s}^{2}}{N^{2}} \sum_{n=1}^{N} \int_{0}^{L_{n}} A_{0} e^{j(2 K z-\omega t)} \mathrm{d} z
\end{aligned}
$$

A new fault to be detected may occur in the feeder line or in one of the $\mathrm{N}$ branches. It is unlikely that, with continuous monitoring, two or more faults occur simultaneously. The case of a fault occurring in the feeder line $\left(d<L_{0}\right)$ is similar to the single fiber scenario where a new fault occurs before an existing known fault:

$$
\begin{aligned}
s(t)= & \int_{0}^{d} A_{0} e^{j(2 K z-\omega t)} \mathrm{d} z+\delta^{2} \int_{d}^{L_{0}} A_{0} e^{j(2 K z-\omega t)} \mathrm{d} z \\
& +\frac{\delta^{2} \delta_{s}^{2}}{N^{2}} \sum_{n=1}^{N} \int_{L_{0}}^{L_{n}} A_{0} e^{j(2 K z-\omega t)} \mathrm{d} z \\
= & \left(1-\delta^{2}\right) \int_{0}^{d} A_{0} e^{j(2 K z-\omega t)} \mathrm{d} z+\delta^{2}\left(1-\frac{\delta_{s}^{2}}{N}\right) \int_{0}^{L_{0}} A_{0} e^{j(2 K z-\omega t)} \mathrm{d} z \\
& +\frac{\delta^{2} \delta_{s}^{2}}{N^{2}} \sum_{n=1}^{N} \int_{0}^{L_{n}} A_{0} e^{j(2 K z-\omega t)} \mathrm{d} z \\
= & \left(1-\delta^{2}\right) \int_{0}^{d} A_{0} e^{j(2 K z-\omega t)} \mathrm{d} z+\delta^{2} s_{0}(t)
\end{aligned}
$$

For the event of a fault occurring in one of the $\mathrm{n}$ branches, the measured signal is described by:

$$
\begin{aligned}
s(t)= & \int_{0}^{L_{0}} A_{0} e^{j(2 K z-\omega t)} \mathrm{d} z+\frac{\delta_{s}^{2}}{N^{2}} \sum_{\substack{n=1 \\
n \neq i}}^{N} \int_{L_{0}}^{L_{n}} A_{0} e^{j(2 K z-\omega t)} \mathrm{d} z \\
& +\frac{\delta_{s}^{2}}{N^{2}}\left[\int_{L_{0}}^{d} A_{0} e^{j(2 K z-\omega t)} \mathrm{d} z+\delta^{2} \int_{d}^{L_{i}} A_{0} e^{j(2 K z-\omega t)} \mathrm{d} z\right] \\
= & \left(1-\frac{\delta_{s}^{2}}{N}\right) \int_{0}^{L_{0}} A_{0} e^{j(2 K z-\omega t)} \mathrm{d} z+\frac{\delta_{s}^{2}}{N^{2}} \sum_{\substack{n=1 \\
n \neq i}}^{N} \int_{0}^{L_{n}} A_{0} e^{j(2 K z-\omega t)} \mathrm{d} z \\
& +\frac{\delta_{s}^{2}}{N^{2}}\left(1-\delta^{2}\right) \int_{0}^{d} A_{0} e^{j(2 K z-\omega t)} \mathrm{d} z+\frac{\delta^{2} \delta_{s}^{2}}{N^{2}} \int_{0}^{L_{i}} A_{0} e^{j(2 K z-\omega t)} \mathrm{d} z \\
= & \left(1-\delta^{2}\right) \frac{\delta_{s}^{2}}{N^{2}} \int_{0}^{d} A_{0} e^{j(2 K z-\omega t)} \mathrm{d} z+s_{0}(t)-\left(1-\delta^{2}\right) \frac{\delta_{s}^{2}}{N^{2}} \int_{0}^{L_{i}} A_{0} e^{j(2 K z-\omega t)} \mathrm{d} z
\end{aligned}
$$

Note that the corresponding reference measured signal $s_{0}(t)$ appears in all of the equations shown here. 